Discussion Paper No. 1027

\title{
SUPPLIER ENCROACHMENT AND \\ RETAILER EFFORT
}

\author{
Noriaki Matsushima \\ Tomomichi Mizuno
}

March 2018

The Institute of Social and Economic Research Osaka University

6-1 Mihogaoka, Ibaraki, Osaka 567-0047, Japan 


\title{
Supplier encroachment and retailer effort*
}

\author{
Noriaki Matsushima ${ }^{\dagger}$ \\ Institute of Social and Economic Research, Osaka University \\ Tomomichi Mizuno \\ Graduate School of Economics, Kobe University
}

March 27, 2018

\begin{abstract}
We propose simple dual-channel models in which an upstream manufacturer trades with a downstream retailer that is able to engage in cost-reducing activities. When the manufacturer determines whether to encroach on the downstream market after observing the retailer's effort level, the threat of manufacturer encroachment can work as a disciplinary device to induce the retailer to aggressively engage in cost reductions, after which the manufacturer refrains from encroaching further on the downstream market. The disciplinary device is more likely to improve consumer welfare and social welfare, although the encroachment itself can harm social welfare.
\end{abstract}

JEL codes: L13, M11, D43.

Keywords: Encroachment, dual-channel model, investment, vertical relation

${ }^{*}$ We thank Takeshi Ebina, the conference participants at the Japan Association for Applied Economics (Tokai University), and the seminar participants at OEIO (Osaka University), Okayama University, and Shinshu University for their valuable discussions and comments. We gratefully acknowledge the financial support of the Japan Society for the Promotion of Science (JSPS), KAKENHI Grant Numbers JP15H03349, JP15H05728, JP17H00984, and JP16K17116. The usual disclaimer applies.

${ }^{\dagger}$ Corresponding author: Noriaki Matsushima, Institute of Social and Economic Research, Osaka University, Mihogaoka 6-1, Ibaraki, Osaka, 567-0047, Japan. Phone: (81)-6-6879-8571. Fax: (81)-6-6879-8583. E-mail: nmatsush@iser.osaka-u.ac.jp

‡Tomomichi Mizuno, Graduate School of Economics, Kobe University, 2-1 Rokkodai, Nada, Kobe, Hyogo, 657-8501, Japan. Phone: (81)-78-803-6802. Fax: (81)-78-803-7289. E-mail: mizuno@econ.kobe-u.ac.jp 


\section{Introduction}

This study considers the impact of manufacturer encroachment on an incumbent monopolistic retailer that is able to engage in cost-reducing activities, as well as on consumer welfare and social welfare. More specifically, we consider the effect on consumer welfare and social welfare of the interaction between the threat of manufacturer encroachment and the retailer's ability to reduce costs.

Supplier encroachment normally harms retailers as a result of the direct competition between existing retailers and the new retailer (i.e., the supplier). ${ }^{1}$ This is particularly serious for small or medium-sized franchisees that trade with national franchisors (see Emerson (2010) for examples in the United States, and Hashimoto (2017) for examples of Japanese convenience stores). On the other hand, it is reasonable for franchisors (from their point of view) to encroach on retail markets when their franchisees perform well or poorly in the relevant markets (Devaraj, Fan, and Kohli, 2002; Rohm and Swaminathan, 2004). This is because when franchisees perform well, there is sufficient room for all parties to earn profits in the retail markets. In contrast, when franchisees perform poorly, an additional retail channel is needed to provide substitutes for the franchisees in the markets. This reasoning implies that franchisees can discourage a franchisor from entering the retail market by being efficient in terms of cost and/or quality through cost-reducing/quality-enhancing activities. In addition, even when encroachment occurs as a result of good performance, the damage it causes is small because the retailers are sufficiently strong in their retail market. ${ }^{2}$ Following this perspective, we can treat franchise encroachment as a disciplinary device for poorly performing franchisees. To avoid supplier encroachment in this case, franchisees can engage in cost-reducing/quality-enhancing activities. The perspective discussed here also implies that franchisors are able to encroach on the retail markets by observing their franchisees' effort levels. However, it would be better for franchisors to decide whether to encroach on the

\footnotetext{
1 However, marketing researchers know that this statement does not always hold in theory (see Arya, Mittendorf, and Sappington, 2007).

2 The latter view is supported by Arya, Mittendorf, and Sappington (2007) from a theoretical point of view. They show that supplier encroachment benefits a monopoly retailer if the retailer is efficient.
} 
retail market without observing their franchisees' effort levels, because this could be a better way to induce franchisees to engage in adequate cost-reducing/quality-enhancing activities. Based on this discussion, we investigate the following research question: how do supplier encroachment and a commitment to (non)encroachment work?

We construct simple dual-channel models in which an upstream manufacturer trades with a downstream retailer that is able to engage in cost-reducing activities. The manufacturer determines whether to encroach on the retail market after observing the retailer's effort level. We also consider models in which the sequence of cost-reduction and encroachment is reversed, as in several works described below. That is, the upstream manufacturer first determines whether to encroach on the market, after which the downstream retailer engages in cost-reducing activities. Here, we compare three alternatives related to manufacturer encroachment: (i) no commitment on whether or not to encroach; (ii) a commitment to encroach; and (iii) a commitment to not encroach. The first alternative is related to the first scenario, and the second and third alternatives are related to the second scenario.

The market structure in our study follows that of Arya, Mittendorf, and Sappington (2007) in the sense that there is one upstream manufacturer and one downstream retailer. The retailer in our study is able to engage in cost-reducing activities by incurring endogenous sunk costs. Observing the retailer's cost reduction, the manufacturer determines whether to encroach on the downstream market. As noted earlier, we also consider situations in which the manufacturer determines whether to encroach on the downstream market before the retailer engages in cost-reducing activities. The marginal cost of the manufacturer's direct channel is assumed to be higher than that of the retailer, as in Arya, Mittendorf, and Sappinton (2007), based on which the manufacturer unilaterally sets the linear wholesale price. We also consider a scenario in which the manufacturer and retailer employ a twopart tariff contract determined through Nash bargaining. Finally, the retailers set their quantities if the manufacturer encroaches on the downstream market as in Arya, Mittendorf, and Sappinton (2007), otherwise, only the retailer monopolistically sets its quantity.

The results are as follows. In the linear contract case, the retailer sets a high invest- 
ment level, which deters the manufacturer from entering the downstream market directly if the manufacturer's direct channel operates at an intermediate level of efficiency. This implies that the threat of manufacturer encroachment can enhance the efficiency of the retailer substantially. However, if the efficiency of the manufacturer's direct channel is high (resp. low), it enters (resp. does not enter) the downstream market directly, which diminishes (resp. maintains) the incentive of the retailer to reduce its costs. Manufacturer encroachment enhances the consumer surplus and the total surplus as a result of strong downstream competition. In addition, if the manufacturer's direct channel is not too inefficient, it has no incentive to commit to a decision on whether or not to encroach; that is, it retains an option to encroach until the retailer actually engages in cost-reduction activities. This outcome is related to situations in which "franchise agreements state that the franchisee has no exclusive territory" (Emerson, 2010, p.234). Interestingly, in this case, the manufacturer's concern over a commitment to encroach is aligned with that of the retailer.

In the case of a two-part tariff contract, the equilibrium decision of the manufacturer on encroachment depends on its bargaining power over the retailer and the efficiency of its direct channel. Specifically, in equilibrium, the manufacturer encroaches on the downstream market if and only if (i) its bargaining power is not sufficiently strong or (ii) its direct channel is significantly inefficient. This is because the benefit of increasing the manufacturer's disagreement payoff dominates the cost of distorting the wholesale price, which controls the retail production. ${ }^{3}$ In contrast, suppose that neither (i) nor (ii) is satisfied. In this case, the retailer sets a high enough investment level such that the manufacturer does not enter the downstream market directly if the efficiency of the direct channel is sufficiently high. Interestingly, the retailer may set a low investment level such that the manufacturer does not enter the retail market directly if the efficiency of the manufacturer in the downstream market is low, but not too low. These two outcomes are similar to that under the linear contract case in that the retailer manipulates its investment level to prevent the manufacturer

\footnotetext{
${ }^{3}$ However, if the manufacturer's bargaining power is sufficiently strong, utilizing the more efficient retailer effectively is better for the manufacturer because it earns enough profit from the fixed fee through bargaining.
} 
from entering the downstream market directly. If the efficiency of the manufacturer's retail channel is at an intermediate level, the retailer sets an optimal investment level as though it does not face a threat of manufacturer encroachment.

In addition to the above results, the manufacturer's decision on whether or not to encroach is almost consistent with what is best for the consumer surplus and the total surplus. This is because the encroachment decision means that the manufacturer controls the retailer's incentive for cost-reducing activities, which enhances the consumer surplus and the total surplus. This finding contrasts sharply with that of Matsushima, Mizuno, and Pan (2018), who show that manufacturer encroachment decreases the total surplus owing to a significant increase in the wholesale price if the efficiency of the manufacturer's retail channel is high. Although we show this counterintuitive outcome in the subgame in which the manufacturer decides whether to open a direct channel before the retailer engages in cost-reducing activities, we show that the outcome is almost not realized in the overall game if we consider the manufacturer's decision on whether or not to commit to encroach. The results in our study and Matsushima, Mizuno, and Pan (2018) imply that if retailers have (resp. do not have) abilities to do such activities, encroachment is beneficial (resp. can be harmful).

Several works are related to ours. Since the pioneering work of Chiang, Chhajed, and Hess (2003), who discuss direct marketing by a manufacturer explicitly, many researchers have investigated market structures in which manufacturers operate in dual channels (e.g., Cattani et al., 2006; Kumar and Ruan, 2006; Yoo and Lee, 2011; Hsiao and Chen, 2013, 2014; Matsui, 2016). Although some of these studies examine an endogenous formation of dual-channel market structures, in other words, manufacturer encroachment (e.g., Arya, Mittendorf, and Sappington, 2007; Mizuno, 2012; Hsiao and Chen, 2013; Li, Xie, and Zhao, 2015; Matsui, 2016; Matsushima, Mizuno, and Pan, 2018; Pan, 2018), they do not consider investments in quality improvement or in cost reduction. In addition, although some works discuss investments in quality improvement or in cost reduction in dual-channel manufacturer models (e.g., Tsay and Agrawal, 2004; Dan, Xu, and Liu, 2012; Pei and Yan, 2015; Yoon, 2016; Chen et al., 2017), they all compare just two kinds of market structures, namely 
those in which a manufacturer does or does not open a direct channel. That is, none of these studies consider the relation between the threat of encroachment and investments by manufacturers and/or retailers, which is the primary focus of our study.

The remainder of the paper proceeds as follows. Section 2 provides the model setting. Section 3 shows the results of the model in which the vertical pair use a linear wholesale price. Then, Section 4 extends the model by incorporating a two-part tariff contract, determined through Nash bargaining. Finally, Section 5 concludes the paper.

\section{Model}

We consider a bilateral monopoly market with one manufacturer $M$ and one retailer $R$. $R$ purchases manufacturing products from $M$ at a linear wholesale price $w$, which it then resells to final consumers at additional marginal cost $c-x_{R}$, where $c$ is a positive constant and $x_{R}$ is $R$ 's non-contractible effort level to reduce its marginal cost. Here, $x_{R}$ is related to operational know-how, managerial efficiency, and so on. Furthermore, $M$ cannot control $x_{R}$ directly by means of a contract. $R$ 's total marginal cost is $c-x_{R}+w$, and $R$ can engage in cost-reducing activities by incurring a cost $x_{R}^{2}$. We also consider a case of a two-part tariff.

We consider $M$ 's option to sell its product directly through its own direct channel, with constant marginal cost $c+c_{M}$, where $c_{M}$ is a positive constant. This implies that $M$ is less efficient than $R$ in terms of handling its product itself in the downstream market. The products distributed through $M$ 's and $R$ 's channels are homogeneous. The inverse demand in the market is given by ${ }^{4}$

$$
p=1-q_{R}-q_{M},
$$

where $q_{i}$ is the quantity supplied by $i=R, M(R$ and $M$ indicate the retailer and manufacturer channel, respectively). If $M$ does not sell its product directly through its own channel,

\footnotetext{
${ }^{4}$ The demand formulation is a special case of the demand system derived from the representative consumer utility $U=\alpha_{R} q_{R}+\alpha_{M} q_{M}-\left(q_{R}^{2}+q_{M}^{2}+2 \theta q_{R} q_{M}\right) / 2$, where $\alpha_{R}=\alpha_{M}=1$ and $\theta=1$ (e.g., Hsiao and Chen, 2013; Chen et al., 2017). Owing to the linearity of the demand system, we can equivalently convert the cost difference between $c-x_{R}$ and $c+c_{M}$ in our model to the quality difference between $\alpha_{R}$ and $\alpha_{M}$, where $R$ 's investment in quality increases $\alpha_{R}$ and the initial level of $\alpha_{R}$ is higher than that of $\alpha_{M}$.
} 
$q_{M}=0$. The profits of $M$ and $R$ are given as

$$
\begin{aligned}
\pi_{M} & =w q_{R}+\left(p-\left(c+c_{M}\right)\right) q_{M}, \\
\pi_{R} & =\left(p-\left(c-x_{R}\right)-w\right) q_{R}-x_{R}^{2} .
\end{aligned}
$$

The consumer surplus, the producer surplus, and the total surplus are defined as follows:

$$
C S \equiv \frac{\left(q_{M}+q_{R}\right)^{2}}{2}, \quad P S \equiv \pi_{M}+\pi_{R}, \quad T S \equiv C S+P S
$$

respectively. We consider the following four-stage game. In stage 1 , the retailer $R$ chooses its investment level $x_{R}$. In stage 2 , the manufacturer $M$ decides whether to open its own direct channel (i.e., encroachment). In stage $3, M$ unilaterally offers its wholesale price $w$ to $R$. In stage $4, R$ and $M$ set quantities $q_{R}$ and $q_{M}$ simultaneously if $M$ opens its own direct channel; otherwise, $R$ sets $q_{R}$ monopolistically. Note that the sequence of stages 1 and 2 follows the scenario motivated in the Introduction. That is, we investigate how the threat of manufacturer encroachment induces $R$ to engage in cost-reducing activities. Then, we also consider a four-stage game in which the sequence of stages 1 and 2 changes; that is, $M$ determines whether to open its own direct channel in stage 1 , and then $R$ chooses $x_{R}$ in stage 2 . The latter approach is followed in several related papers, as described in the Introduction.

\section{Equilibrium}

We need to consider two subgames after stage 2: (i) $M$ does not open its own direct channel (it does not encroach); and (ii) $M$ opens its own direct channel (it encroaches). We denote the former and the latter case as $N$ and $E$, respectively.

\subsection{The manufacturer does not encroach $($ case $N)$}

In stage 4 , the maximization problem of $R$ is $\max _{q_{R}}\left(1-q_{R}-\left(c-x_{R}\right)-w\right) q_{R}-x_{R}^{2}$, leading to $q_{R}^{N}\left(x_{R}, w\right)=\left(1-c+x_{R}-w\right) / 2$. In stage 3 , anticipating the outcome, $M$ maximizes $w q_{R}^{N}\left(x_{R}, w\right)$, leading to $w^{N}\left(x_{R}\right)=\left(1-c+x_{R}\right) / 2$ and $q_{R}^{N}\left(x_{R}, w^{N}\right)=\left(1-c+x_{R}\right) / 4$. The 
resulting profits of $R$ and $M$ in stage 3 are respectively given as

$$
\begin{aligned}
\pi_{R}^{N}\left(x_{R}\right) & =\frac{\left(1-c+x_{R}\right)^{2}}{16}-x_{R}^{2}, \\
\pi_{M}^{N}\left(x_{R}\right) & =\frac{\left(1-c+x_{R}\right)^{2}}{8} .
\end{aligned}
$$

\subsection{The manufacturer encroaches (case $E$ )}

In stage 4 , the maximization problems of $R$ and $M$ in stage 4 are $\max _{q_{R}}\left(1-q_{R}-q_{M}-(c-\right.$ $\left.\left.x_{R}\right)-w\right) q_{R}-x_{R}^{2}$ and $\max _{q_{M}} w q_{R}+\left(1-q_{R}-q_{M}-\left(c+c_{M}\right)\right) q_{M}$, respectively, leading to ${ }^{5}$

$$
\begin{aligned}
& q_{R}^{E}\left(x_{R}, w\right)= \begin{cases}\frac{1-c+c_{M}+2 x_{R}-2 w}{3} & \text { if } w>x_{R}-\left((1-c)-2 c_{M}\right), \\
\frac{1-c+x_{R}-w}{2} & \text { if } w \leq x_{R}-\left((1-c)-2 c_{M}\right),\end{cases} \\
& q_{M}^{E}\left(x_{R}, w\right)= \begin{cases}\frac{1-c-2 c_{M}-x_{R}+w}{3} & \text { if } w>x_{R}-\left((1-c)-2 c_{M}\right), \\
0 & \text { if } w \leq x_{R}-\left((1-c)-2 c_{M}\right) .\end{cases}
\end{aligned}
$$

In stage 3 , anticipating the outcome, $M$ maximizes $w q_{R}^{E}\left(x_{R}, w\right)+\left(1-q_{R}^{E}\left(x_{R}, w\right)-\right.$ $\left.q_{M}^{E}\left(x_{R}, w\right)-\left(c+c_{M}\right)\right) q_{M}^{E}\left(x_{R}, w\right)$, leading to

$$
w^{E}\left(x_{R}\right)= \begin{cases}\frac{5(1-c)-c_{M}+4 x_{R}}{10} & \text { if } x_{R}<\frac{5(1-c)-7 c_{M}}{2} \equiv x_{R}^{l}, \\ x_{R}-\left(1-c-2 c_{M}\right) & \text { if } x_{R}^{l} \leq x_{R}<3(1-c)-4 c_{M} \equiv x_{R}^{h} \\ \frac{1-c+x_{R}}{2} & \text { if } x_{R}^{h} \leq x_{R} .\end{cases}
$$

The resulting profits of $R$ and $M$ in stage 3 are respectively given as

$$
\begin{aligned}
& \pi_{R}^{E}\left(x_{R}\right)= \begin{cases}\frac{4\left(c_{M}+x_{R}\right)^{2}}{25}-x_{R}^{2} & \text { if } x_{R}<x_{R}^{l}, \\
\left(1-c-c_{M}\right)^{2}-x_{R}^{2} & \text { if } x_{R}^{l} \leq x_{R}<x_{R}^{h}, \\
\frac{\left(1-c+x_{R}\right)^{2}}{16}-x_{R}^{2} & \text { if } x_{R}^{h} \leq x_{R},\end{cases} \\
& \pi_{M}^{E}\left(x_{R}\right)= \begin{cases}\frac{\left(1-c-c_{M}\right)^{2}}{4}+\frac{\left(c_{M}+x_{R}\right)^{2}}{5} & \text { if } x_{R}<x_{R}^{l}, \\
\left(1-c-c_{M}\right)\left(x_{R}-\left(1-c-2 c_{M}\right)\right) & \text { if } x_{R}^{l} \leq x_{R}<x_{R}^{h}, \\
\frac{\left(1-c+x_{R}\right)^{2}}{8} & \text { if } x_{R}^{h} \leq x_{R} .\end{cases}
\end{aligned}
$$

\footnotetext{
${ }^{5}$ We do not explicitly mention the possibility in which $q_{R}^{E}\left(x_{R}, w\right)=0$. This is because $R$ is more efficient than $M$ 's direct channel, implying that $M$ does not shut down $R$ using a prohibitively high $w$.
} 
This result implies that $R$ can be active if and only if it has a cost advantage over $M$ 's direct channel, which is given by $c_{M}+x_{R}$. In addition, when $x_{R} \geq x_{R}^{h}$, the outcome is equivalent to that in case $N$.

\subsection{Opening $M$ 's direct channel: Stage 2}

To simplify the exposition, we define

$$
z \equiv \frac{c_{M}}{1-c}
$$

The decision of $M$ to open its own direct channel depends on the investment level $x_{R}$. Specifically, $M$ opens its own channel if and only if $\pi_{M}^{E}\left(x_{R}\right)>\pi_{M}^{N}\left(x_{R}\right)$; that is,

$$
x_{R}<\bar{x}_{R} \equiv \frac{(1-c)\{(5-\sqrt{10})-(8-\sqrt{10}) z\}}{3} .
$$

\subsection{Cost-reducing investment: Stage 1}

Using the outcomes in (5), (7), and (9), we obtain the objective of $R$ in stage 1 as follows:

$$
\pi_{R}\left(x_{R}\right) \equiv \begin{cases}\pi_{R}^{E}\left(x_{R}\right)=\frac{4\left((1-c) z+x_{R}\right)^{2}}{25}-x_{R}^{2} & \text { if } x_{R}<\bar{x}_{R}, \\ \pi_{R}^{N}\left(x_{R}\right)=\frac{\left(1-c+x_{R}\right)^{2}}{16}-x_{R}^{2}, & \text { if } x_{R} \geq \bar{x}_{R} .\end{cases}
$$

Here, $\bar{x}_{R}<0$ if and only if $z>(5-\sqrt{10}) /(8-\sqrt{10}) \simeq 0.380$. Within this range of $z, x_{R}^{h}>\bar{x}_{R}$. Solving the maximization problem $\max _{x_{R}} \pi_{R}\left(x_{R}\right)$, we obtain the following proposition.

Proposition 1 The optimal investment level of $R$ is given as: ${ }^{6}$

$$
x_{R}^{*}= \begin{cases}x_{R}^{E} \equiv \frac{4(1-c) z}{21} & \text { if } z \leq 0.181, \\ \bar{x}_{R}=\frac{(1-c)\{(5-\sqrt{10})-(8-\sqrt{10}) z\}}{3} & \text { if } 0.181<z \leq 0.339, \\ x_{R}^{N} \equiv \frac{1-c}{15} & \text { if } z>0.339 .\end{cases}
$$

\footnotetext{
${ }^{6}$ The exact values of 0.181 and 0.339 are $(847-2 \sqrt{70(8 \sqrt{10}-13)}-224 \sqrt{10}) /(1327-280 \sqrt{10})$ and $(24-5 \sqrt{10}) /(5(8-\sqrt{10}))$, respectively.
} 
In this scenario, $M$ opens its own direct channel if and only if $z \leq 0.181$. When $z>0.339$, the profits of $M$ and $R$, the consumer surplus, and the total surplus are constant with an increase in $z$. When $0.181<z \leq 0.339$, the profit of $M$ decreases with $z$, the profit of $R$ increases with $z$, the consumer surplus decreases with $z$, and the total surplus is concave with respect to $z$. When $z \leq 0.181$, the profit of $M$ decreases with $z$, the profit of $R$ increases with $z$, and the consumer surplus and the total surplus decrease with $z$.

Here, we explain the outcome graphically although an explicit derivation is available in Appendix A1. A notable feature of the outcome is that $R$ sets significantly higher $x_{R}$ so as not to induce $M$ to encroach when $z$ lies in the intermediate range of values in (11) (see Figure 1). Thus, R's effort to avoid encroachment enhances $M$ 's profit substantially, leading to a non-monotonic relation between $M$ 's profit and $z$ (see Figure 1).

[Figure 1 about here]

From Figure 1, we find that the wholesale price $w^{*}$ on the domain of smaller $z$, where encroachment occurs, is smaller than that on the domain of larger $z$, where encroachment does not occur. This is reminiscent of the main mechanism in Arya, Mittendorf, and Sappington (2007). On the domain of smaller $z$, the direct effect of welfare improvement from encroachment, as a result of a reduction in wholesale prices and an increase in competition, dominates the indirect effect of welfare deterioration resulting from a reduction in investment. Thus, encroachment improves the consumer surplus and the total surplus.

\subsection{Cost-reduction after encroachment}

Here, observing the decision of $M$ to open its own direct channel, $R$ determines its level of cost reduction in stage 2 . For the case of no encroachment, from (10) and (11), the investment 
level is $x_{R}^{N}=(1-c) / 15$. In addition, for the case of encroachment, the investment level is

$$
x_{R}^{E}= \begin{cases}\frac{4(1-c) z}{21} & \text { if } z \leq 0.677 \\ \frac{(1-c)(5-7 z)}{2}\left(=x_{R}^{l}\right) & \text { if } 0.677<z \leq 0.714 \\ 0 & \text { if } 0.714<z \leq 0.742, \\ \frac{1-c}{15} & \text { if } 0.742<z\end{cases}
$$

Substituting the outcome in stage 2 into (6) and (8), we have the profit of $M$ under the two cases:

$$
\begin{aligned}
& \pi_{M}^{N}\left(x_{R}^{N}\right)= \frac{32(1-c)^{2}}{225} \\
& \pi_{M}^{E}\left(x_{R}^{E}\right)= \begin{cases}\frac{125 z^{2}(1-c)^{2}}{441}+\frac{(1-c)^{2}(1-z)^{2}}{4} & \text { if } z \leq 0.677, \\
\frac{3(1-z)^{2}(1-c)^{2}}{2} & \text { if } 0.677<z \leq 0.714, \\
(1-z)(2 z-1)(1-c)^{2} & \text { if } 0.714<z \leq 0.742, \\
\frac{32(1-c)^{2}}{225} & \text { if } 0.742<z .\end{cases}
\end{aligned}
$$

Comparing the two values in (13) and (14), we obtain the following proposition. ${ }^{7}$

Proposition 2 Manufacturer $M$ opens its own direct channel if

$$
z<0.336 \text { or } 0.601<z<0.692 \text {. }
$$

If the condition in (15) is satisfied, $R$ 's optional investment level is $x_{R}^{E}$ in (12); otherwise, it is $x_{R}^{N}=(1-c) / 15$. If $M$ does not encroach, the profits of $M$ and $R$, the consumer surplus, and the total surplus are constant with a change in $z$. When $M$ encroaches, the profit of $M$ decreases in $z$ for $z<0.336$ and for $z \in(0.677,0.692)$, and increases in $z$ for $z \in(0.601,0.677]$. The profit of $R$ decreases in $z$ for $z \in(0.689,0.692)$, and increases in $z$ for $z<0.336$ or $z \in(0.601,0.689)$. The consumer surplus decreases in $z$ for $z<0.336$ or $z \in(0.601,0.692)$. Lastly, the total surplus decreases in $z$ for $z<0.336$ or $z \in(0.677,0.692)$, and increases in $z$ for $z \in(0.601,0.677)$.

\footnotetext{
7 The exact values of $0.336, \quad 0.601,0.677, \quad 0.689$, and 0.692 are $7(315-2 \sqrt{1987}) / 4705$, $7(315+2 \sqrt{1987}) / 4705,21 / 31,31 / 45$, and $(45-8 \sqrt{3}) / 45$, respectively. Note that $M$ 's retailer is inactive if and only if $z>21 / 31$.
} 
The equilibrium outcome is summarized in Figure 2. A notable feature of the outcome is that for $z \in(0.601,0.692)$, encroachment reduces the wholesale price substantially (Arya, Mittendorf, and Sappington, 2007), inducing $R$ to invest aggressively. As a result, both $M$ and $R$ benefit from $M$ 's encroachment. Moreover, on this range of $z$, $(0.601,0.692)$, the wholesale price reduction means the incentive for $R$ to invest is enhanced by encroachment. That is, the existence of $R$ 's investment opportunity reinforces the benefits of encroachment described in Arya, Mittendorf, and Sappington (2007).

[Figure 2 about here]

\subsection{Commitment problem: linear pricing}

We consider the endogenous decision of whether or not to commit to encroachment/nonencroachment. The sequence of the game is as follows. Initially, $M$ decides whether to commit to encroachment or non-encroachment. At this stage, $M$ has three alternatives: (i) $M$ commits to non-encroachment, and then it never opens its own direct channel; (ii) $M$ commits to encroachment, and then it immediately opens its own direct channel; (iii) $M$ does not commit to either encroachment or non-encroachment, and it determines whether or not to encroach after observing $R$ 's effort level, $x_{R}$. When $M$ chooses the first alternative, the subgame is the non-encroachment case in Section 3.5. When $M$ chooses the second alternative, the subgame is the encroachment case in Section 3.5. When $M$ chooses the third alternative, the subgame is the non-commitment case in Section 3.4.

To identify $M$ 's incentive to commit to encroachment/non-encroachment, we compare the outcomes in Sections 3.4 and 3.5 by merging Figures 1 and 2. The comparison is summarized in Figure 3.

[Figure 3 about here]

The outcome is summarized as the following proposition.

Proposition 3 If $z<0.601, M$ does not have an incentive to commit to encroach. That is, it retains the option to encroach until $R$ actually engages in cost-reduction activities; otherwise, it commits to encroaching on the retail market. 
The former outcome is related to situations in which "franchise agreements state that the franchisee has no exclusive territory" (Emerson, 2010, p.234). Interestingly, the decision of $M$ on whether to commit is consistent with $R$ 's interests. In particular, in the intermediate range of $z,[0.181,0.339], R$ and $M$ both prefer the non-commitment on encroachment in Section 3.4 to the commitment on encroachment in Section 3.5. This is because the former outcome allows $R$ to monopolize the retail market, owing to $R$ 's significant effort, whereas the latter leads to a duopoly, irrespective of the retailer's effort. For the upper range of $z$, [0.601, 0.692), as discussed in Section 3.5, a commitment on encroachment benefits both $M$ and $R$, owing to the wholesale price reduction, which is expected by $R$ in the investment stage and encourages it to engage in cost-reduction activities.

From the viewpoint of the consumer surplus and the total surplus, the decision by $M$ on whether to commit is almost consistent with what is best for the total surplus. However, it is not consistent with what is best for the consumer surplus for the intermediate range of $z$, $[0.181,0.339]$. The latter inconsistency stems from the retail monopoly resulting from nonencroachment and the wholesale price increase, which offsets the benefit from the enhanced effort of $R$.

\section{Two-part tariff}

Now, we return to the basic model by allowing two-part tariff contracts. The difference between the setting here and that in the basic model lies in the third stage. Specifically, we consider the following four-stage game. In stage $1, R$ chooses its investment level $x_{R}$. In stage $2, M$ decides whether to open its own direct channel. In stage $3, M$ and $R$ negotiate a two-part tariff contract, $(w, f)$, through Nash bargaining, where $w$ is the wholesale price and $f$ is the fixed payment to $M$. Then, $\alpha$ is the bargaining power of $R$ over $M$. This is the difference between this and the basic model. In stage $4, R$ and $M$ set quantities $q_{R}$ and $q_{M}$ simultaneously if $M$ opens its own direct channel; otherwise, $R$ sets $q_{R}$ monopolistically. Note that we also consider a four-stage game in which the sequence of stages 1 and 2 is reversed; that is, $M$ determines whether or not to encroach in stage 1, after which $R$ chooses 
$x_{R}$ in stage 2 .

We need to consider two subgames after stage 2: (i) $M$ does not open its own direct channel; (ii) $M$ encroaches on the downstream market. We denote the former and the latter cases as $N T$ and ET, respectively.

\subsection{The manufacturer does not encroach (case $N T$ )}

In stage 4 , the outcome is $q_{R}^{N}\left(x_{R}, w\right)=\left(1-c+x_{R}-w\right) / 2$. In stage 3 , anticipating the outcome, $M$ and $R$ negotiate a two-part tariff, in which their disagreement profits are both zero (excluding $R$ 's sunk investment cost). The bargaining outcome is given as

$$
w^{N T}=0, \quad f^{N T}\left(x_{R}\right)=\frac{(1-\alpha)\left(1-c+x_{R}\right)^{2}}{4} .
$$

Avoiding the double-marginalization problem (by setting $w^{N T}=0$ ), $M$ and $R$ simply split the downstream monopoly profit $\left(1-c+x_{R}\right)^{2} / 4$ through Nash bargaining. The resulting profits of $R$ and $M$ in stage 3 are respectively given as

$$
\begin{aligned}
& \pi_{R}^{N T}\left(x_{R}\right)=\frac{\alpha\left(1-c+x_{R}\right)^{2}}{4}-x_{R}^{2}, \\
& \pi_{M}^{N T}\left(x_{R}\right)=\frac{(1-\alpha)\left(1-c+x_{R}\right)^{2}}{4} .
\end{aligned}
$$

\subsection{The manufacturer encroaches (case ET)}

In stage 4, the outcome is the same as that in the linear pricing case. We restate it here.

$$
\begin{aligned}
& q_{R}^{E}\left(x_{R}, w\right)= \begin{cases}\frac{(1-c)(1+z)+2 x_{R}-2 w}{3} & \text { if } w>x_{R}-(1-c)(1-2 z) \equiv \bar{w}\left(x_{R}\right), \\
\frac{1-c+x_{R}-w}{2} & \text { if } w \leq \bar{w}\left(x_{R}\right),\end{cases} \\
& q_{M}^{E}\left(x_{R}, w\right)= \begin{cases}\frac{(1-c)(1-2 z)-x_{R}+w}{3} & \text { if } w>\bar{w}\left(x_{R}\right), \\
0 & \text { if } w \leq \bar{w}\left(x_{R}\right) .\end{cases}
\end{aligned}
$$

The resulting profits of $R$ and $M$ are $\pi_{R}^{E}\left(x_{R}, w\right)=\left(q_{R}^{E}\left(x_{R}, w\right)\right)^{2}-f-x_{R}^{2}$ and $\pi_{M}^{E}\left(x_{R}, w\right)=$ $\left(q_{M}^{E}\left(x_{R}, w\right)\right)^{2}+w q_{R}^{E}\left(x_{R}, w\right)+f$, respectively.

In stage 3, anticipating the outcome, $M$ and $R$ negotiate a two-part tariff. In contrast to case $N T, M$ has a disagreement profit in which the negotiation breaks down. This is 
$\pi_{M}^{O}=(1-c)^{2}(1-z)^{2} / 4$, which is realized if $M$ is the monopolist after the breakdown of the negotiation. The bargaining problem is given as

$$
\max _{w, f} \alpha \log \left[\pi_{R}^{E}\left(x_{R}, w\right)-\left(-x_{R}^{2}\right)\right]+(1-\alpha) \log \left[\pi_{M}^{E}\left(x_{R}, w\right)-\pi_{M}^{O}\right]
$$

The bargaining outcome is given $\operatorname{as}^{8}$

$$
\begin{aligned}
& w^{E T}\left(x_{R}\right)= \begin{cases}\frac{(1-c)(1-5 z)-4 x_{R}}{2} & \text { if } x_{R}<\frac{(1-c)(1-3 z)}{2} \equiv K_{L}, \\
-(1-c)(1-2 z)+x_{R} & \text { if } K_{L} \leq x_{R}<(1-c)(1-2 z) \equiv K_{H}, \\
0 & \text { if } K_{H} \leq x_{R},\end{cases} \\
& f^{E T}\left(x_{R}\right)= \begin{cases}(4-\alpha)\left((1-c) z+x_{R}\right)^{2} . & \text { if } x_{R}<K_{L}, \\
\frac{(1-c)(1-z)\left((1-c)(4-\alpha-(4-5 \alpha) z)+4 \alpha x_{R}\right)}{4} & \text { if } K_{L} \leq x_{R}<K_{H}, \\
\frac{(1-\alpha)\left(1-c+x_{R}\right)^{2}+\alpha(1-c)^{2}(1-z)^{2}}{4} & \text { if } K_{H} \leq x_{R} .\end{cases}
\end{aligned}
$$

Owing to the encroachment by $M, w^{E T}\left(x_{R}\right)$ non-monotonically changes with $x_{R}$. For $x_{R}<$ $K_{L}, w^{E T}\left(x_{R}\right)$ decreases because they need to shift a retail quantity from $M$ 's direct channel to $R$, which is more efficient. At $x_{R}=K_{L}, q_{M}^{E}\left(x_{R}, w^{E T}\left(x_{R}\right)\right)$ becomes zero, which implies that halting $M$ 's direct channel is optimal. In addition, at $x_{R}=K_{L}$, the realized negative wholesale price is in itself inefficient from the industry viewpoint, because it induces an excessive supply by $R$. For $x_{R} \in\left(K_{L}, K_{H}\right), w^{E T}\left(x_{R}\right)$ increases because it becomes easier to halt $M$ 's direct channel as $R$ 's efficiency improves through the increase in $x_{R}$. The resulting profits of $R$ and $M$ in stage 3 are respectively given as

$$
\begin{gathered}
\pi_{R}^{E T}\left(x_{R}\right)= \begin{cases}\alpha\left((1-c) z+x_{R}\right)^{2}-x_{R}^{2} . & \text { if } x_{R}<K_{L}, \\
\frac{(1-c)(1-z)\left(-\left((1-c)(1-5 z)+4 x_{R}\right)\right.}{4}-x_{R}^{2} & \text { if } K_{L} \leq x_{R}<K_{H}, \\
\frac{\alpha\left((1-c) z+x_{R}\right)\left((1-c)(2-z)+x_{R}\right)}{4}-x_{R}^{2} & \text { if } K_{H} \leq x_{R},\end{cases} \\
\pi_{M}^{E T}\left(x_{R}\right)= \begin{cases}\frac{(1-c)^{2}(1-z)^{2}+4(1-\alpha)\left((1-c) z+x_{R}\right)^{2}}{4} . & \text { if } x_{R}<K_{L}, \\
\frac{(1-c)(1-z)\left((1-c)(\alpha+(4-5 \alpha) z)+4(1-\alpha) x_{R}\right)}{4} & \text { if } K_{L} \leq x_{R}<K_{H}, \\
\frac{(1-\alpha)\left(1-c+x_{R}\right)^{2}+\alpha(1-c)^{2}(1-z)^{2}}{4} & \text { if } K_{H} \leq x_{R} .\end{cases}
\end{gathered}
$$

\footnotetext{
${ }^{8}$ Because $\pi_{M}^{O}$ does not depend on either $w$ or $f$, they simply maximize their joint profits by controlling $w$.
} 


\subsection{Opening $M$ 's direct channel: Stage 2}

The decision by $M$ to open its own direct channel depends on the investment level $x_{R}$. Specifically, $M$ opens its direct channel if and only if $\pi_{M}^{E T}\left(x_{R}\right)>\pi_{M}^{N T}\left(x_{R}\right)$. This inequality holds if and only if

$$
\begin{cases}x_{R}<\bar{x}_{R}^{L} \equiv \frac{(1-c)((1-\alpha)(1-4 z)-J(1-z))}{3(1-\alpha)} & \\ \text { or } x_{R}>\bar{x}_{R}^{H} \equiv \frac{(1-c)((1-\alpha)(1-2 z)-(1-z) \sqrt{\alpha(1-\alpha)})}{1-\alpha} & \text { if (i) } \alpha \in[0,1 / 5], \\ x_{R}<\bar{x}_{R}^{L} \text { or } x_{R}>\bar{x}_{R}^{M} \equiv \frac{(1-c)((1-\alpha)(1-4 z)+J(1-z))}{3(1-\alpha)} & \text { if (ii) } \alpha \in(1 / 5,1 / 4], \\ \text { for any } x_{R} & \text { if (iii) } \alpha \in(1 / 4,1],\end{cases}
$$

where $J \equiv \sqrt{(1-4 \alpha)(1-\alpha)}$.

Note that for $\alpha \in[0,1 / 5], \bar{x}_{R}^{H}>K_{L}$ and $\bar{x}_{R}^{M}>K_{L}$; for $\alpha \in[1 / 5,1 / 4], \bar{x}_{R}^{H}<K_{L}$ and $\bar{x}_{R}^{M}<K_{L}$.

\subsection{Cost-reducing investment: Stage 1}

For the three cases (i) $\alpha \in[0,1 / 5]$, (ii) $\alpha \in(1 / 5,1 / 4]$, and (iii) $\alpha \in(1 / 4,1]$, using the outcomes in (16), (18), and (20), we obtain the objective of $R$ in stage 1 as follows:

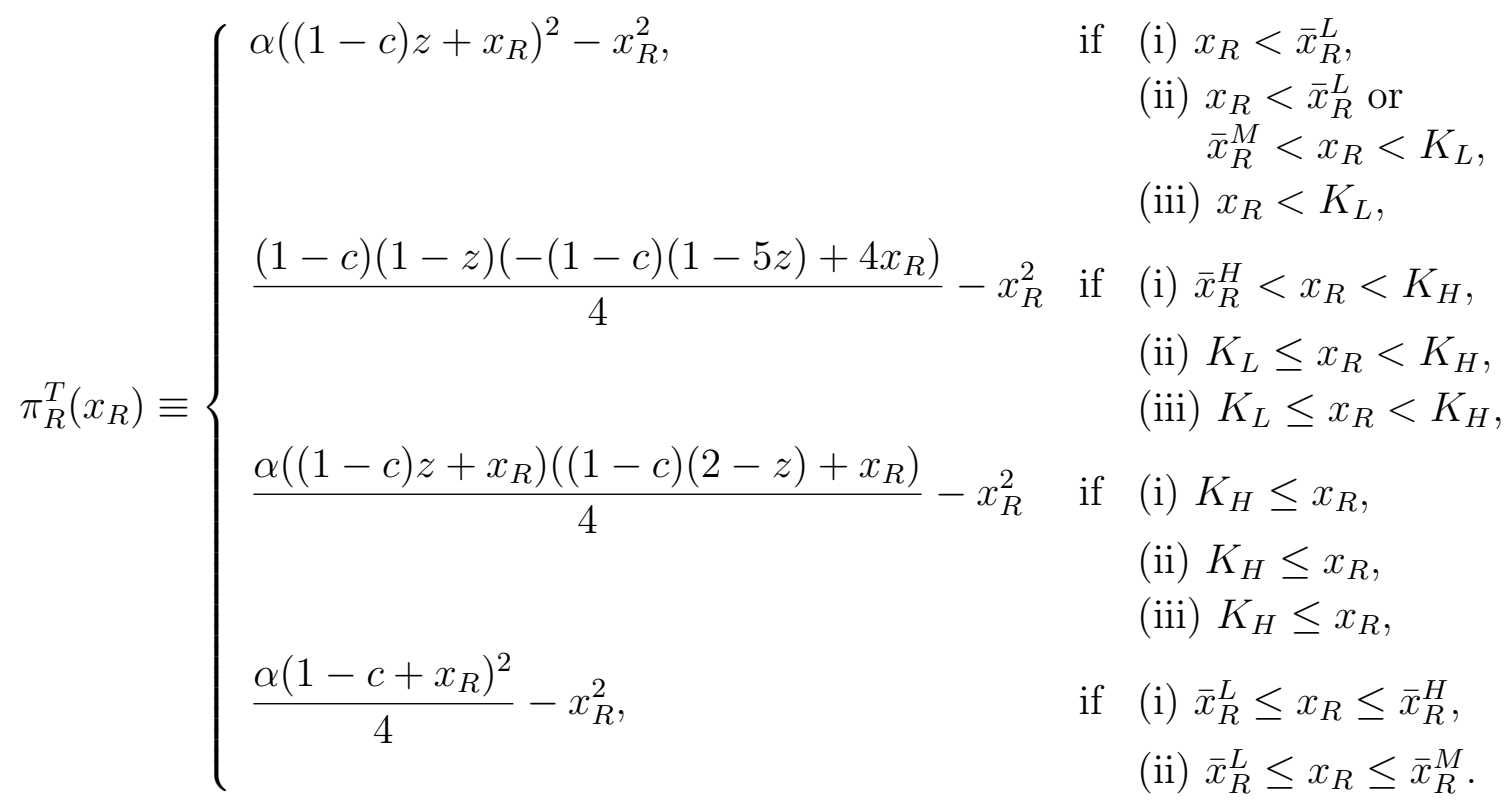

Solving the maximization problem $\max _{x_{R}} \pi_{R}^{T}\left(x_{R}\right)$, we obtain the following proposition. 
Proposition 4 The optimal investment level of $R$ is given as:

$$
x_{R}^{T *}= \begin{cases}\bar{x}_{R}^{L} & \text { if } \alpha \leq 1 / 4 \text { and } z \leq z^{L}, \\ x_{R}^{N T} \equiv \frac{\alpha(1-c)}{4-\alpha} & \text { if } \alpha \leq 1 / 5 \text { and } z^{L}<z<z^{H}, \text { or } \\ & 1 / 5<\alpha \leq 1 / 4 \text { and } z^{L}<z<z^{M} \\ \bar{x}_{R}^{H} & \text { if } \alpha \leq 1 / 5 \text { and } z^{H} \leq z \leq z^{x R H}, \\ \bar{x}_{R}^{M} & \text { if } 1 / 5<\alpha \leq 1 / 4 \text { and } z^{M} \leq z \leq z^{x R M}, \\ x_{R}^{E T} & \text { otherwise, }\end{cases}
$$

where

$$
\begin{aligned}
& \left(\frac{\alpha(1-c) z}{1-\alpha} \equiv x_{R}^{E T L}, \quad \text { if } 0.247<\alpha \leq 1 / 4 \text { and } z^{x R M} \leq z<z_{L}^{E T}\right. \text { or, } \\
& 1 / 4<\alpha \text { and } z<z_{L}^{E T}, \\
& x_{R}^{E T} \equiv\left\{\begin{aligned}
\frac{\alpha(1-c)(1-z)}{2} \equiv x_{R}^{E T M}, \quad \text { if } \alpha \leq 1 / 5 \text { and } z^{x R H} \leq z<z_{H}^{E T}, \text { or } \\
1 / 5<\alpha \leq 0.247 \text { and } z^{x R M} \leq z<z_{H}^{E T}, \text { or }
\end{aligned}\right. \\
& 0.247<\alpha \leq 1 / 4 \text { and } z_{L}^{E T} \leq z<z_{H}^{E T} \text {, or } \\
& 1 / 4<\alpha \text { and } z_{L}^{E T} \leq z<z_{H}^{E T}, \\
& \frac{\alpha(1-c)}{4-\alpha} \equiv x_{R}^{E T H}, \quad \text { if } z_{H}^{E T} \leq z \text { for any } \alpha, \\
& z^{L} \equiv \frac{4-5 \alpha+4 \alpha^{2}-4 J}{(4-\alpha)(5-4 \alpha)}, z^{M} \equiv \frac{4-5 \alpha+4 \alpha^{2}+4 J}{(4-\alpha)(5-4 \alpha)}, \\
& z^{H} \equiv \frac{8-16 \alpha+5 \alpha^{2}-4 \sqrt{\alpha(1-\alpha)}}{(4-\alpha)(4-5 \alpha)}, z_{L}^{E T} \equiv \frac{1-\alpha}{3-\alpha}, \quad z_{H}^{E T} \equiv \frac{2-\alpha}{4-\alpha}, \\
& z^{x R M} \equiv \frac{1+J}{5-4 \alpha}, \quad z^{x R H} \equiv \frac{2-3 \alpha-\sqrt{\alpha(1-\alpha)}}{4-5 \alpha} .
\end{aligned}
$$

$M$ opens its own direct channel if and only if the condition in (23) holds.

The outcome in (22) and M's decision on whether to encroach are summarized in Figure 4.

[Figure 4 about here]

Next, we review how $M$ 's encroachment decision influences the wholesale price. If $M$ does not encroach, the wholesale price is zero, irrespective of $z$. Otherwise, it decreases with $z$ monotonically whenever $M$ 's direct channel supplies a positive quantity, and is negative at the minimum value of $z$ such that the direct channel just stops its supply. This relation is one of the key factors in our model. 
When $\alpha \leq 1 / 4$ and $z \leq z^{L}, R$ sets $x_{R}=\bar{x}_{R}^{L}$, which is larger than $x_{R}^{N T}$, to avoid having $M$ encroach (see $D_{S}$ and $N$ in $x_{R} /(1-c)$ in Figure 5). This aggressive investment enhances $M$ 's profit, but reduces $R$ 's profit (see (22) and Figure 5). When $\alpha \leq 1 / 5$ and $z^{L}<z<z^{H}$, or $\alpha \in(1 / 5,1 / 4]$ and $z^{L}<z<z^{M}$, $R$ 's investment level, $x_{R}^{N T}$, does not depend on $z$, but does depend on $\alpha$, which has a positive effect on the profit share of $R$ in the negotiation. The higher profit share of $R$ means that a larger $\alpha$ has a positive impact on $R$ 's investment level (see $x_{R} /(1-c)$ in Figure 5). ${ }^{9}$ When $\alpha \leq 1 / 5$ and $z^{H} \leq z \leq z^{x R H}$ (resp. $\alpha \in(1 / 5,1 / 4]$ and $z^{M} \leq z \leq z^{x R M}$ ), interestingly, $R$ sets $x_{R}=\bar{x}_{R}^{H}$ (resp. $x_{R}=\bar{x}_{R}^{M}$ ), which is lower than $x_{R}^{N T}$, to avoid inducing $M$ to encroach, diminishing the profits of both $M$ and $R$ (see $D_{W}$ in $x_{R} /(1-c)$ in Figure 5). This is because $M$ would encroach on the downstream market to encourage $R$ to produce more together with a negative wholesale price if $R$ became sufficiently efficient by setting $x_{R}=x_{R}^{N T}$.

When the condition in (23) holds, $M$ decides to encroach. $R$ is more useful when $M$ 's efficiency in the downstream market is lower. To use $R$ effectively, $M$, with a higher $z$, sets a lower wholesale price, which encourages $R$ 's production. Anticipating the lower wholesale price, $R$ sets a higher investment level (see $E$ in $x_{R} /(1-c)$ in Figure 5). The resulting (gross) profit of $R$ is partially transferred to $M$ through the fixed payment. In fact, $R$ 's profit increases with $z$ monotonically, whereas $M$ 's profit turns upward at an intermediate value of $z$, owing to $R$ 's stronger investment incentive (see the thin lines (the case of $\alpha=0.28$ ) in $\pi_{M} /(1-c)^{2}$ and $\pi_{R} /(1-c)^{2}$ in Figure 5$)$.

[Figure 5 about here]

\subsection{Cost-reduction after encroachment}

Here, observing $M$ 's decision on whether to open its own direct channel, $R$ determines its level of cost reduction in stage 2. The investment levels in the cases of non-encroachment

\footnotetext{
${ }^{9}$ Here, $x_{R}^{N T}$ is the same as $x_{R}^{E T}$ in the case of $z_{H}^{E T} \leq z$, although these are derived from $\max _{x_{R}} \pi_{R}^{T}\left(x_{R}\right)$ in the fourth and third cases, respectively, in equation (21).
} 
and encroachment are respectively as follows:

$$
x_{R}^{N T} \equiv \frac{\alpha(1-c)}{4-\alpha}, \quad x_{R}^{E T} \equiv \begin{cases}x_{R}^{E T L}, & \text { if } z<z_{L}^{E T}, \\ x_{R}^{E T M}, & \text { if } z_{L}^{E T} \leq z<z_{H}^{E T}, \\ x_{R}^{E T H}, & \text { if } z_{L}^{E T} \leq z\end{cases}
$$

Substituting $x_{R}^{E T}$ in (25) into (17) and (19), we have the profit of $M$ under the two cases

$$
\begin{aligned}
\pi_{M}^{N T}\left(x_{R}^{N T}\right) & =\frac{4(1-\alpha)(1-c)^{2}}{(4-\alpha)^{2}}, \\
\pi_{M}^{E T}\left(x_{R}^{E T}\right) & = \begin{cases}\frac{(1-c)^{2}\left((1-\alpha)(1-z)^{2}+4 z^{2}\right)}{4(1-\alpha)}, & \text { if } z<z_{L}^{E T,}, \\
\frac{(1-c)^{2}(1-z)\left(\alpha(3-2 \alpha)+\left(4-7 \alpha+2 \alpha^{2}\right) z\right)}{4}, & \text { if } z_{L}^{E T} \leq z<z_{H}^{E T}, \\
\frac{(1-c)^{2}\left(\alpha(4-\alpha)^{2}(1-z)^{2}+16(1-\alpha)\right)}{4(4-\alpha)^{2}}, & \text { if } z_{L}^{E T} \leq z .\end{cases}
\end{aligned}
$$

Comparing the two values in (26) and (27), we obtain the following proposition. ${ }^{10}$

Proposition 5 Manufacturer $M$ opens its own direct channel if and only if

$$
\begin{aligned}
& \alpha \geq 0.263, \quad \text { or } \\
& \alpha<0.263 \text { and } z<\frac{(4-\alpha)(1-\alpha)-2 \sqrt{(1-\alpha)\left(4-16 \alpha+3 \alpha^{2}\right)}}{(5-\alpha)(4-\alpha)} \equiv \bar{z}_{M}^{L} \text { or } \\
& z>\max \left[\frac{(4-\alpha)(1-\alpha)+2 \sqrt{(1-\alpha)\left(4-16 \alpha+3 \alpha^{2}\right)}}{(5-\alpha)(4-\alpha)},\right. \\
& \left.\frac{(4-\alpha)(2-\alpha)(1-2 \alpha)-2 \sqrt{\alpha(1-\alpha)\left(4+\alpha-\alpha^{2}\right)}}{(4-\alpha)\left(4-7 \alpha+2 \alpha^{2}\right)}\right] \equiv \bar{z}_{M}^{H} .
\end{aligned}
$$

Figure 6 shows the condition in which $M$ encroaches on the downstream market.

[Figure 6 about here]

For $\alpha>0.263$, encroachment gives a stronger bargaining position (a larger disagreement payoff) to $M$, although it also causes a distortion in the retail distribution. For $\alpha<0.263$, depending on the value of $z, M$ opens its own direct channel. The impact of $M$ 's decision on encroachment depends on the efficiency of $M, z$, because $z$ influences the wholesale price under encroachment. If $z$ is large, encroachment encourages $R$ to engage in cost-reducing

\footnotetext{
10 The exact value of 0.263 is $2(4-\sqrt{13}) / 3$.
} 
activities, owing to the negative wholesale price offered by $M$. If $z$ is an intermediate value, non-encroachment encourages $R$ to engage in cost-reducing activities owing to its secured monopoly position. If $z$ is small, $M$ encroaches on the retail market simply because encroachment increases its disagreement payoff. However, when $z$ is small, from the viewpoint of $R$ 's investment incentive, there is a discrepancy between the realized channel structure (encroachment) and the preferred structure (non-encroachment). The discrepancy and a sufficiently large wholesale price, in which $z$ is small, harms $R$ 's profit and the consumer surplus and the total surplus, as in the main analysis. Fortunately, except for the case in which $z$ is small, the equilibrium channel structure is acceptable from an efficiency viewpoint.

[Figure 7 about here]

Welfare reducing encroachment Compared to the case in which $M$ commits to not encroach, commitment to encroachment harms the consumer surplus and the total surplus if the efficiency of $M$ is high enough because $M$ sets a significantly higher wholesale price. Figure 7 shows the possibility. The curve on $z \in[0,0.061]$ (resp. $z \in[0,0.085]$ ) in the case of $\alpha=0.18$ (resp. $\alpha=0.22$ ) represents the outcome in which $M$ encroaches. The flat line-segment on $z \in[0.061,0.269]$ (resp. $z \in[0.085,0.24]$ ) in the case of $\alpha=0.18$ (resp. $\alpha=0.22)$ represents the outcome in which $M$ commits to not encroach and the outcome does not depend on the value of $z$ because $M$ 's direct channel is inactive. We find that $C S /(1-c)^{2}$ and $T S /(1-c)^{2}$ for sufficiently small $z$ are lower than those in which $M$ does not encroach. We also find that $w /(1-c)$ for sufficiently small $z$ is significantly higher than that in which $M$ does not encroach $(w=0)$. Although this welfare reducing encroachment is derived in Matsushima, Mizuno, and Pan (2018), the negative result almost does not appear if we consider $M$ 's decision on commitment to encroachment or non-encroachment as in Section 3.6. 


\subsection{Commitment problem: two-part tariff}

Here, as in Section 3.6, to identify M's incentive to commit to encroachment or to nonencroachment, we compare the outcomes in Sections 4.4 and 4.5. We need to compare M's three alternatives: (i) $M$ commits to non-encroachment, and then it immediately opens its own direct channel; (ii) $M$ commits to encroachment, and then it never opens its own direct channel; (iii) $M$ does not commit to either encroachment or non-encroachment, and it determines whether or not to encroach after observing $R$ 's effort level, $x_{R}$.

Given $R$ 's effort level, the consumer surplus in the non-encroachment case and that in the encroachment case are given as

$$
\begin{aligned}
& C S^{N T}\left(x_{R}\right)= \frac{\left(1-c+x_{R}\right)^{2}}{8}, \\
& C S^{E T}\left(x_{R}\right)= \begin{cases}\frac{\left((1-c)(1+z)+2 x_{R}\right)^{2}}{8} & \text { if } x_{R}<K_{L}, \\
\frac{(1-c)^{2}(1-z)^{2}}{2} & \text { if } K_{L} \leq x_{R}<K_{H}, \\
\frac{\left((1-c)(2-z)+x_{R}\right)^{2}}{18} & \text { if } K_{H} \leq x_{R} .\end{cases}
\end{aligned}
$$

The resulting total surplus in the non-encroachment case and that in the encroachment case are $T S^{N T}\left(x_{R}\right)=\pi_{R}^{N T}\left(x_{R}\right)+\pi_{M}^{N T}\left(x_{R}\right)+C S^{N T}\left(x_{R}\right)$ and $T S^{E T}\left(x_{R}\right)=\pi_{R}^{E T}\left(x_{R}\right)+\pi_{M}^{E T}\left(x_{R}\right)+$ $C S^{E T}\left(x_{R}\right)$, respectively. Let be $z^{C S}$ and $z^{T S}$ the larger roots of the quadratic equations $C S^{N T}\left(\bar{x}_{R}^{L}\right)-C S^{E T}\left(x_{R}^{E T L}\right)=0$ and $T S^{E T}\left(x_{R}^{E T L}\right)-T S^{N T}\left(x_{R}^{N T}\right)=0$, with respect to $z$, respectively. After some calculus, we obtain the condition that $M$ 's choice for the three alternatives:

Proposition $6 M$ does not commit to either encroachment or non-encroachment if

$$
\begin{cases}z \leq \bar{z}_{M}^{H} \text { or } z \geq z^{x R H} & \text { when } \alpha \in[0,1 / 5) \\ z \leq \bar{z}_{M}^{H} \text { or } z \geq z^{x R M} & \text { when } \alpha \in[1 / 5,1 / 4) \\ z \leq \bar{z}_{M}^{L} \text { or } z \geq \bar{z}_{M}^{H} & \text { when } \alpha \in[1 / 4,0.263) \\ \text { for any } z & \text { when } \alpha \in(0.263,1]\end{cases}
$$

(see the white, blue, and gray areas in Figure 8), otherwise, it commits to either encroachment or non-encroachment on the retail market (the read area in Figure 8). More concretely, given 
that the condition in (29) does not hold, $M$ commits to encroachment if $\alpha<1 / 4$, otherwise, $M$ commits to non-encroachment. $M$ 's choice for the three alternatives is not consistent with $R$ 's interest if $\bar{z}_{M}^{L}<z<z^{L}$ or $\bar{z}_{M}^{H}<z<z^{x R H}$ when $0<\alpha<1 / 5$, or $\bar{z}_{M}^{L}<z<z^{L}$ or $\bar{z}_{M}^{H}<z<z^{x R M}$ when $1 / 5 \leq \alpha<1 / 4$ (see the gray and red area without shade or dots in Figure 8). M's choice for the three alternatives is not consistent with what is best for the consumer surplus if $z^{C S}<z<\bar{z}_{M}^{L}$ when $0<\alpha<1 / 4$, or $\bar{z}_{M}^{L}<z<\bar{z}_{M}^{H}$ when for $1 / 4 \leq \alpha<0.263$ (see the blue area with shade and the red area with shade or dots in Figure 8). M's choice for the three alternatives is not consistent with what is best for the total surplus if $z^{T S}<z<\bar{z}_{M}^{H}$ when $1 / 4 \leq \alpha<0.2626$ (see the red area with shade in Figure 8). $M$ 's choice for the three alternatives is consistent with what is best for $R$, the consumer surplus, and the total surplus if

$$
\begin{cases}z \leq z^{C S}, z^{L} \leq z \leq \bar{z}_{M}^{H}, \text { or } z \geq z^{x R H} & \text { when } \alpha \in[0,1 / 5), \\ z \leq z^{C S}, z^{L} \leq z \leq \bar{z}_{M}^{H}, \text { or } z \geq z^{x R M} & \text { when } \alpha \in[1 / 5,1 / 4), \\ z \leq \bar{z}_{M}^{L} \text { or } z \geq \bar{z}_{M}^{H} & \text { when } \alpha \in[1 / 4,0.263), \\ \text { for any } z & \text { when } \alpha \in(0.263,1] .\end{cases}
$$

Figure 8 summarizes the conditions in Proposition 6.

[Figure 8 about here]

Except the red area with shade on $\alpha \in[1 / 4,0.263), M$ 's choice for the three alternatives is consistent with what is best for the total surplus. When $\alpha<1 / 4, M$ 's choice for the alternatives enhances $R$ 's incentive to set a higher effort level which is beneficial to the total surplus.

In Figure 9, the thin solid line on smaller $z$ in the case of $\alpha=0.15$ is related to the investment enhancing effect $\left(\right.$ see $x_{R} /(1-c)$ ), and the thick dashed line on $z \in[0.136,0.176]$ in the case of $\alpha=0.26$ is related to the negative effect of commitment to non-encroachment. This is quite different from the result of welfare reducing encroachment in Section 4.5.

[Figure 9 about here] 


\section{Conclusion}

This study considers the impact of manufacturer encroachment on a monopolistic incumbent retailer that is able to engage in cost-reducing activities, as well as on the consumer surplus and the total surplus. We construct simple dual-channel models showing when an upstream manufacturer encroaches on the retail market after observing the retailer's effort level. We consider a linear contract and a two-part tariff contract. In particular, we take into account the bargaining power of each agent in the latter case.

We also consider models in which the sequence of cost-reduction and encroachment is reversed, as in the related studies listed in the Introduction. That is, the upstream manufacturer first determines whether or not to encroach, after which the downstream retailer engages in cost-reducing activities.

In addition, we compare three alternatives on manufacturer encroachment: (i) a noncommitment on encroachment, (ii) a commitment to encroach, and (iii) a commitment to not encroach. The first alternative is related to the first scenario, and the second and third alternatives are related to the second scenario.

The results are as follows. In the linear contract case, the retailer sets a high investment level which prevents the manufacturer from entering the downstream market directly if the efficiency of the manufacturer in the retail market is at an intermediate level. If the efficiency of the manufacturer in the downstream market is high (resp. low), it enters (resp.

does not enter) the downstream market directly, which reduces (resp. keeps) the incentive of the retailer to engage in cost-reduction activities. Manufacturer encroachment enhances the consumer surplus and the total surplus as a result of strong downstream competition. In addition, if the manufacturer's direct channel is not too inefficient, it does not have an incentive to commit on whether to encroach; that is, it retains the option to encroach until the retailer actually engages in cost-reduction activities. Interestingly, the manufacturer's concern over a commitment to encroach is aligned with that of the retailer. That is, the retailer prefers the manufacturer's non-commitment to a decision to encroach.

In the two-part tariff contract case, the equilibrium decision of the manufacturer on 
encroachment depends on its bargaining power over the retailer and on the efficiency of its direct channel. Specifically, in equilibrium, the manufacturer encroaches on the downstream market if and only if (i) its bargaining power is not sufficiently strong or (ii) its direct channel is significantly inefficient. When neither (i) nor (ii) is satisfied, the retailer sets a high investment level, which prevents the manufacturer from entering the downstream market directly if the efficiency of the manufacturer's direct channel is high. Interestingly, the retailer may set a low investment level to prevent the manufacturer from entering the retail market directly if the efficiency of the manufacturer in the downstream market is low (but not too low). These two outcomes are similar to those in the linear contract case in that the retailer manipulates its investment level so as not to induce the manufacturer to enter the downstream market directly. If the efficiency of the manufacturer's direct channel is at an intermediate level, the retailer sets an optimal investment level as though there is no threat of manufacturer encroachment.

In addition, in the two-part tariff contract case, if the manufacturer's direct channel is not inefficient, it does not have an incentive to commit on whether to encroach. That is, it retains the option to encroach until the retailer actually engages in cost-reduction activities. Other than in an intermediate range of manufacturer efficiency, the manufacturer's concern over the commitment to encroach is almost aligned with that of the retailer. Moreover, the manufacturer's encroachment decision is almost consistent with what is best for the consumer surplus and the total surplus. This is because the decision on whether to commit to encroach enables the manufacturer to control the retailer's incentive for cost-reducing activities, which enhances both the consumer surplus and the total surplus. This finding contrasts sharply with that of Matsushima, Mizuno, and Pan (2018), who show that manufacturer encroachment decreases the total surplus as a result of a significant increase in the wholesale price if the efficiency of manufacturer's retail channel is high. Although we also shows this counterintuitive outcome in Section 4.5, we show that it is almost not realized in the overall game if we take into account cost-reducing activities and the manufacturer's decision on whether to commit to encroach, as in Section 4.6. 
Here, we have considered a monopolistic buyer-supplier relationship. As an extension of our model, we can consider a bilateral duopoly with cost asymmetry in the downstream level. Then, if it is not too complex, we could extend this second model further to include two incumbent downstream firms that engage in cost reduction. These scenarios are both left for future research.

\section{References}

Arya, Anil, Brian Mittendorf, David E.M. Sappington, 2007. The bright side of supplier encroachment. Marketing Science 26(5), 651-659.

Cattani, Kyle, Wendell Gilland, Hans Sebastian Heese, Jayashankar Swaminathan, 2006. Boiling frogs: Pricing strategies for a manufacturer adding a direct channel that competes with the traditional channel. Production and Operations Management 15(1), $40-56$.

Chen, Jingxian, Liang Liang, Dong-Qing Yao, Shengnan Sun, 2017. Price and quality decisions in dual-channel supply chains. European Journal of Operational Research 259(3), 935-948.

Chiang, Wei-yu Kevin, Dilip Chhajed, James D. Hess, 2003. Direct marketing, indirect profits: A strategic analysis of dual-channel supply-chain design. Management Science 49(1), 1-20.

Dan, Bin, Guangye Xu, Can Liu, 2012. Pricing policies in a dual-channel supply chain with retail services. International Journal of Production Economics 139(1), 312-320.

Devaraj, Sarv, Ming Fan, and Rajiv Kohli, 2002. Antecedents of B2C channel satisfaction and preference: Validating e-commerce metrics. Information Systems Research 13(3), 316-333.

Hashimoto, Yoko, 2017. Kombini-ōnā no roudousha-sei: Furanchaizu-keiyaku to roudouhou (Owners of convenience stores as workers: Franchise contracts and labor law). Ni- 
hon Roudou Kenkyu Zasshi (Japanese Journal of Labour Research) 678, 29-40 (in Japanese).

Hsiao, Lu, Ying-Ju Chen, 2013. The perils of selling online: Manufacturer competition, channel conflict, and consumer preferences. Marketing Letters 24(3), 277-292.

Hsiao, Lu, Ying-Ju Chen, 2014. Strategic motive for introducing Internet channels in a supply chain. Marketing Letters 24(3), 277-292.

Kumar, Nanda, Ranran Ruan, 2006. On manufacturers complementing the traditional retail channel with a direct online channel. Quantitative Marketing and Economics 4(3), 289-323.

Li, Tingting, Jinxing Xie, Xiaobo Zhao, 2015. Supplier encroachment in competitive supply chains. International Journal of Production Economics 165, 120-131.

Matsui, Kenji, 2016. Asymmetric product distribution between symmetric manufacturers using dual-channel supply chains. European Journal of Operational Research 248(2), 646-657.

Matsushima, Noriaki, Tomomichi Mizuno, Cong Pan, 2018. A manufacturer's incentive to open its direct channel and its impact on welfare. ISER Discussion Paper No. 1026. Osaka University.

Mizuno, Tomomichi, 2012. Direct marketing in oligopoly. Journal of Economics \&3 Management Strategy 21(2), 373-397.

Pan, Cong, 2018. Supplier encroachment and consumer welfare: Upstream firm's opportunism and multichannel distribution. ISER Discussion Paper No. 1020. Osaka University. Available at SSRN: https://papers.ssrn.com/abstract=3124026.

Pei, Zhi, Ruiliang Yan, 2015. Do channel members value supportive retail services? Why? Journal of Business Research 68, 1350-1358. 
Rohm, Andrew J., Vanitha Swaminathan, 2004. A typology of online shoppers based on shopping motivations. Journal of Business Research 57(7), 748-757.

Tsay, Andy A., Narendra Agrawal, 2004. Channel conflict and coordination in the ecommerce age. Production and Operations Management 13(1), 93-110.

Yoo, Weon Sang, Eunkyu Lee, 2011. Internet channel entry: A strategic analysis of mixed channel structures. Marketing Science 30(1), 29-41.

Yoon, Dae-Hee, 2016. Supplier encroachment and investment spillovers. Production and Operations Management 25(11), 1839-1854. 

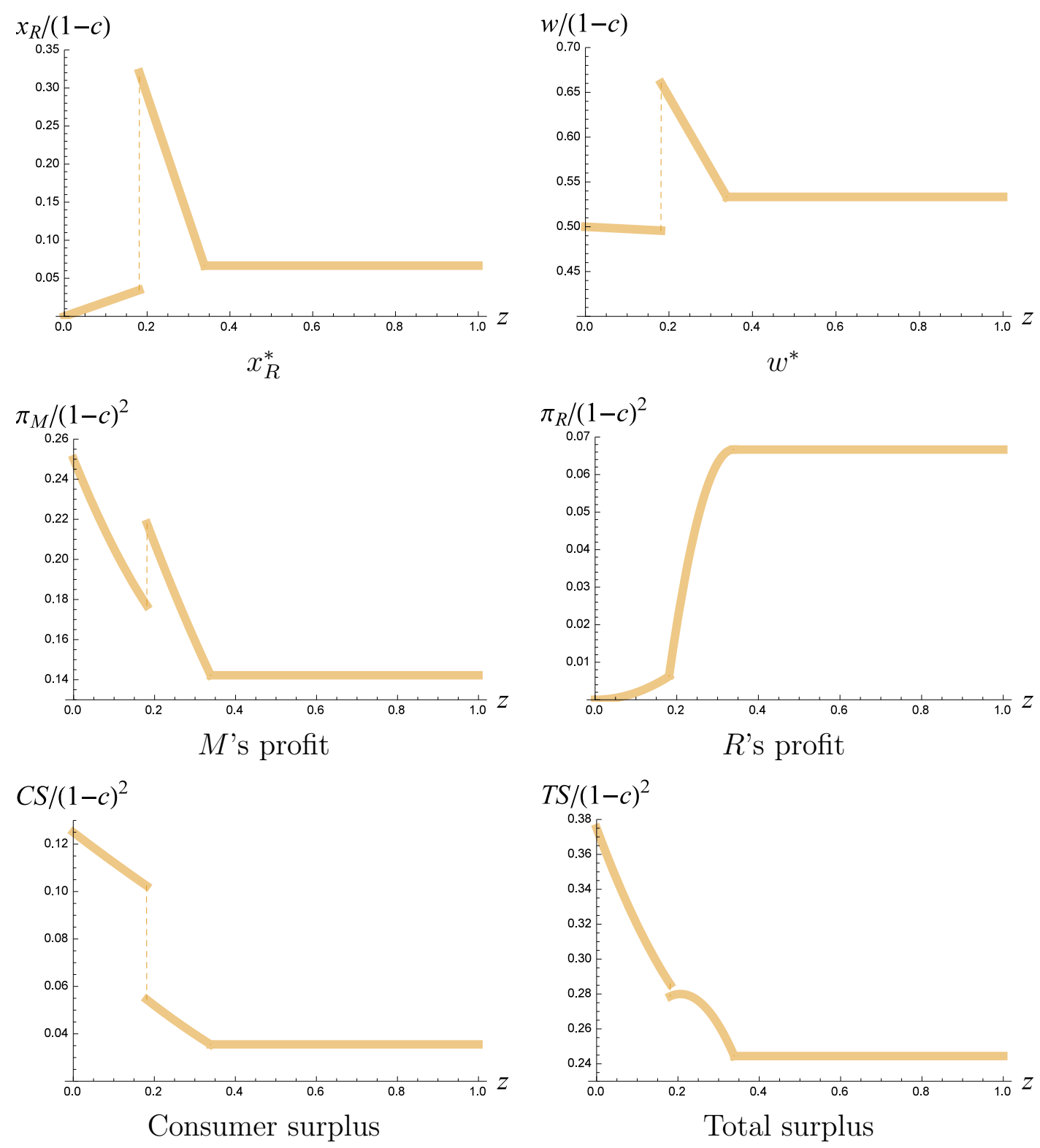

Figure 1: The outcome under a linear contract (R\&D-Encroachment) Note: Horizontal axis is $c_{M} /(1-c)$ 

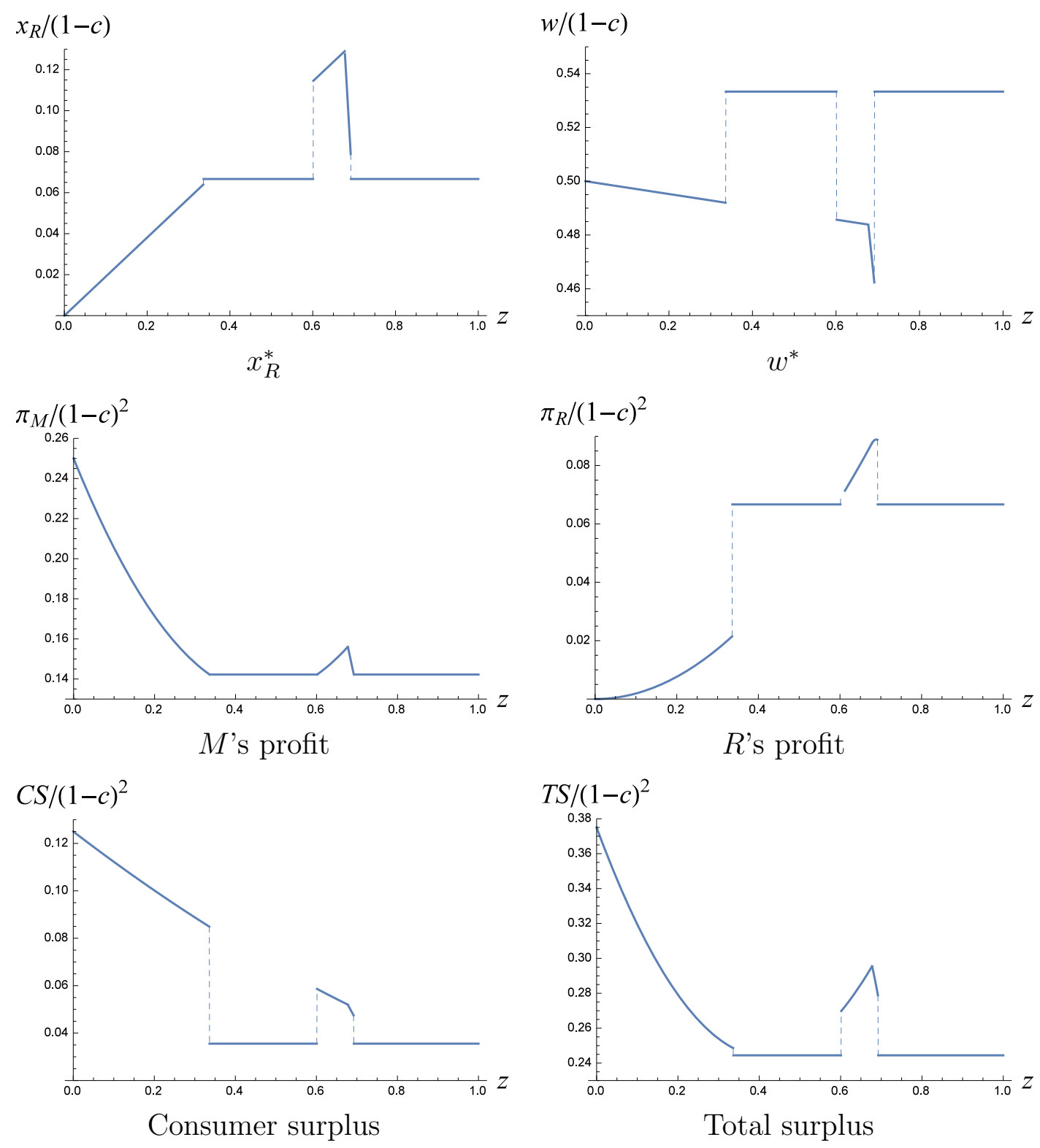

Figure 2: The outcome under a linear contract (Encroachment-R\&D) Note: Horizontal axis is $c_{M} /(1-c)$ 

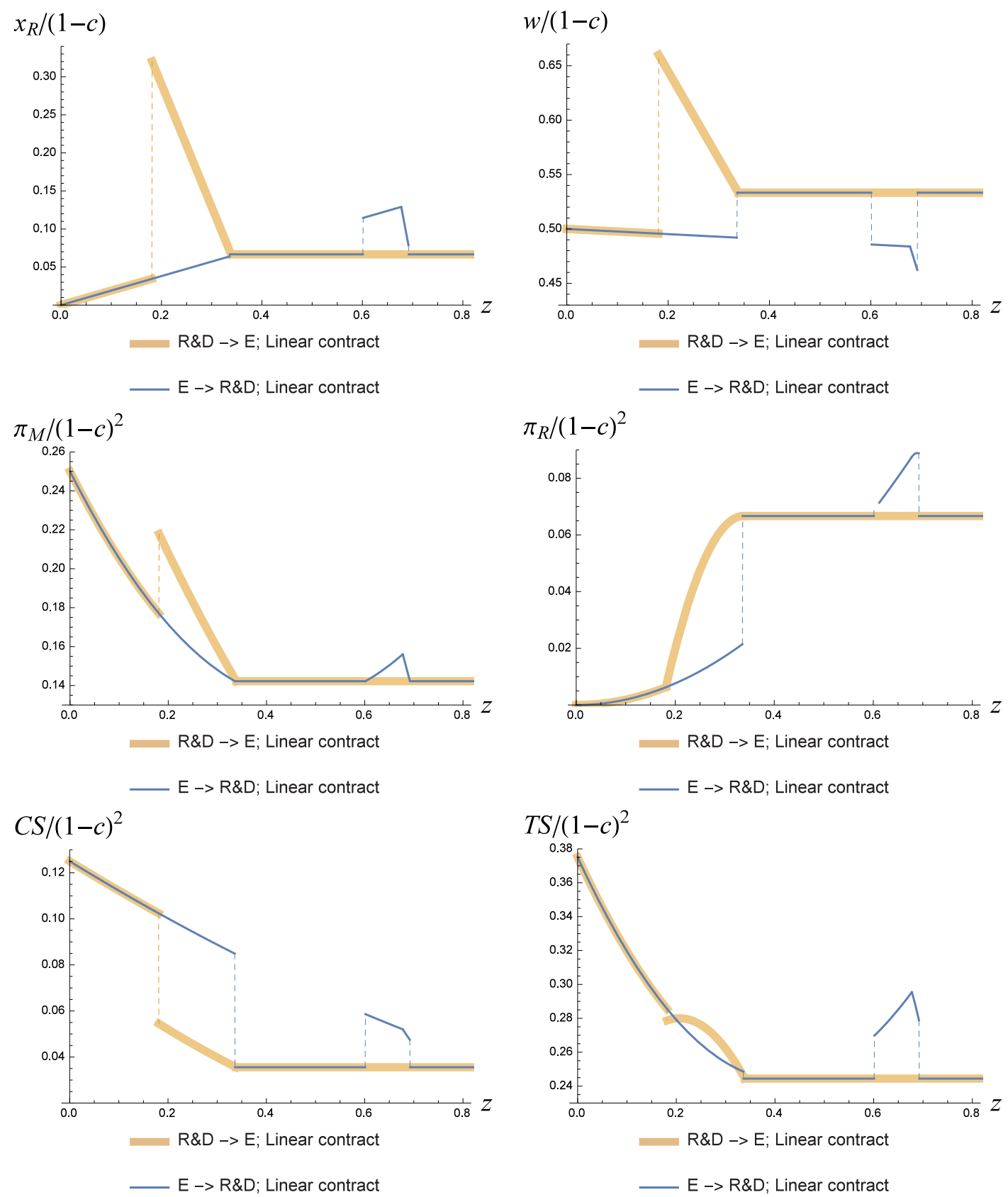

Figure 3: Comparison of the two scenarios under a linear contract Note: Horizontal axis is $c_{M} /(1-c)$ 


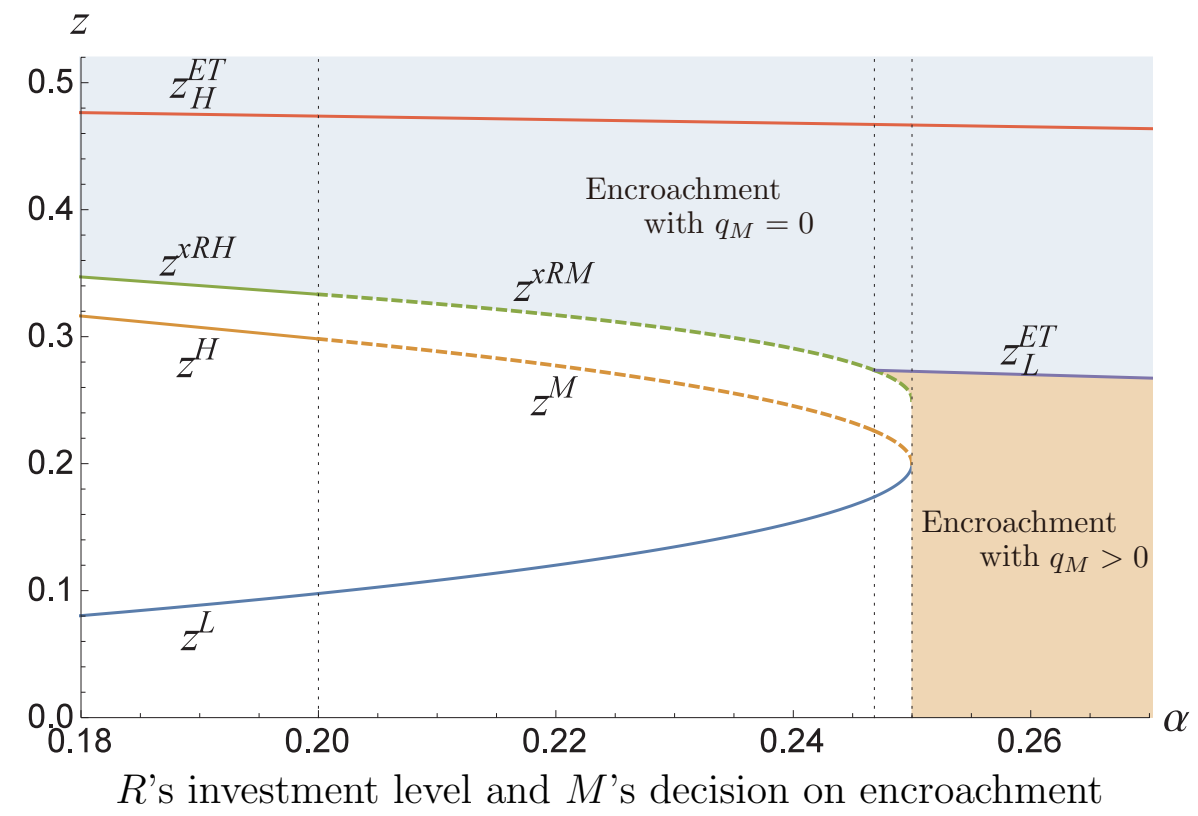

Figure 4: Investment level and encroachment decision (R\&D-Encroachment) 


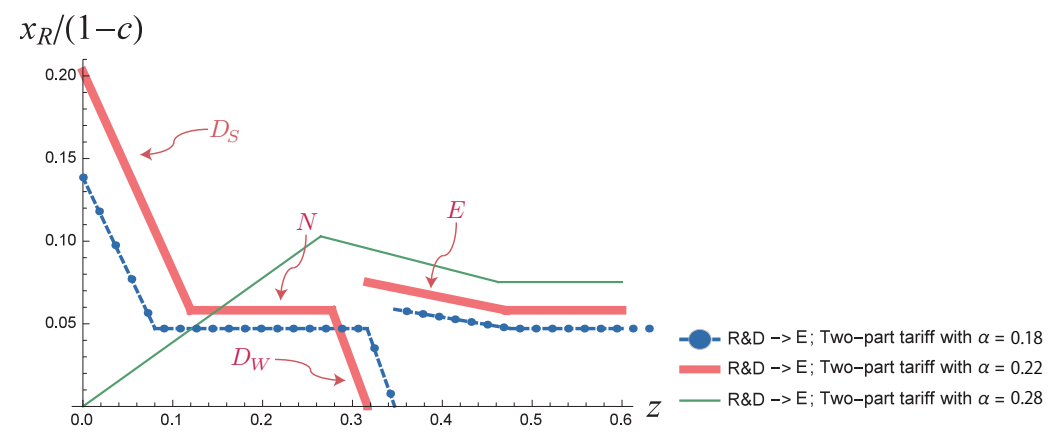

$w /(1-c)$

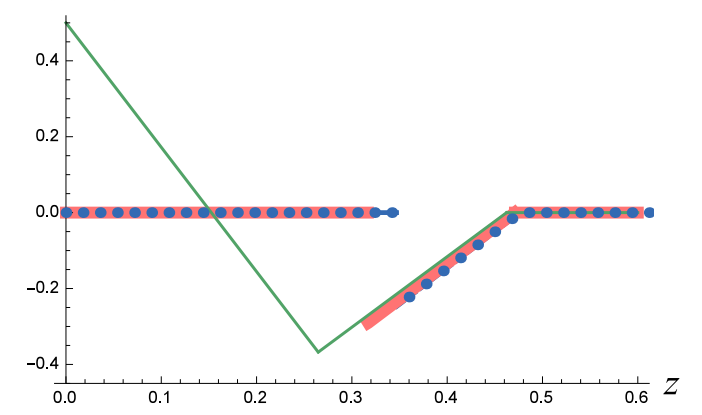

$f /(1-c)^{2}$
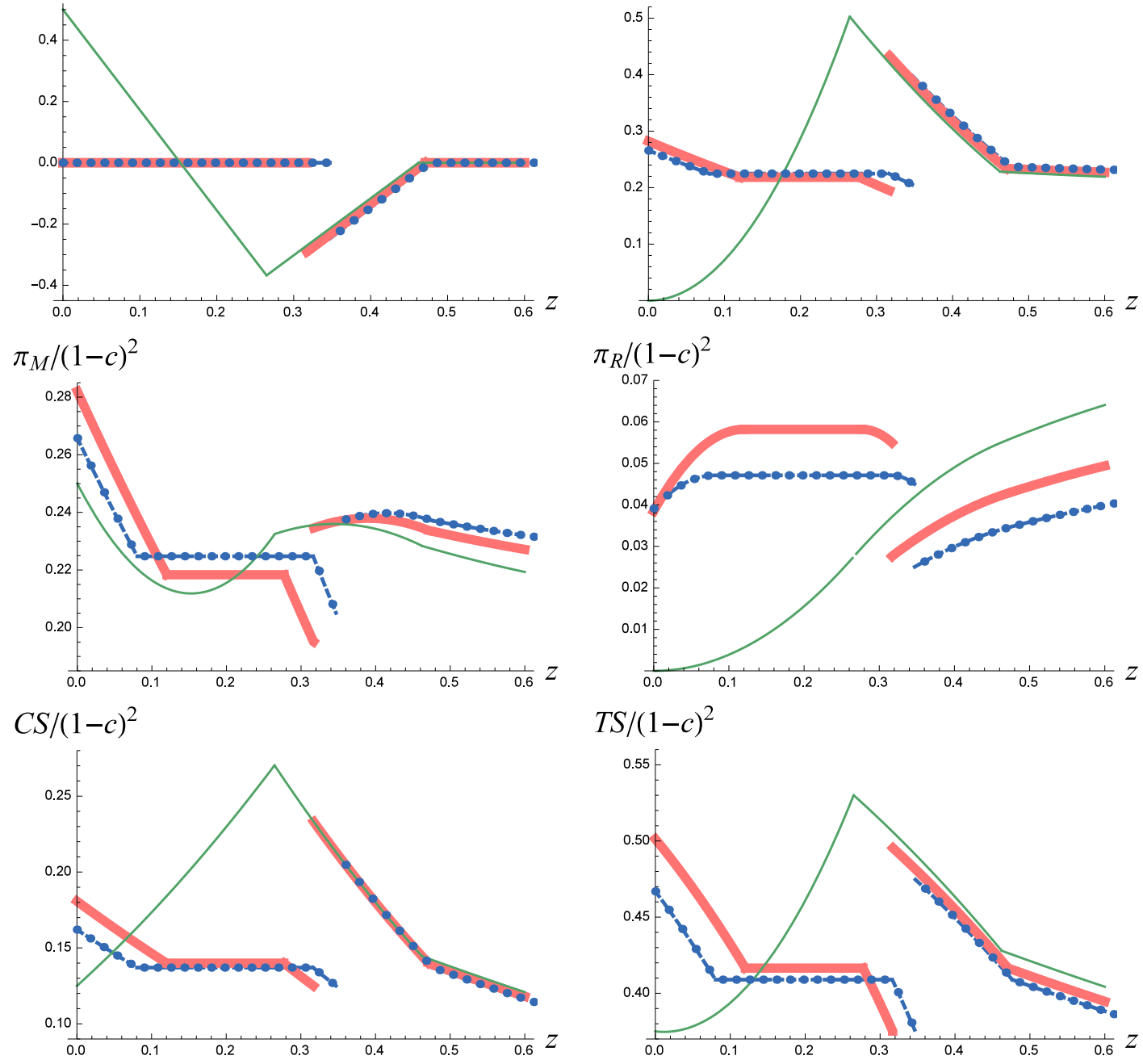

Figure 5: The outcomes under a two-part tariff (R\&D-Encroachment)

Note: Horizontal axis is $c_{M} /(1-c)$ 


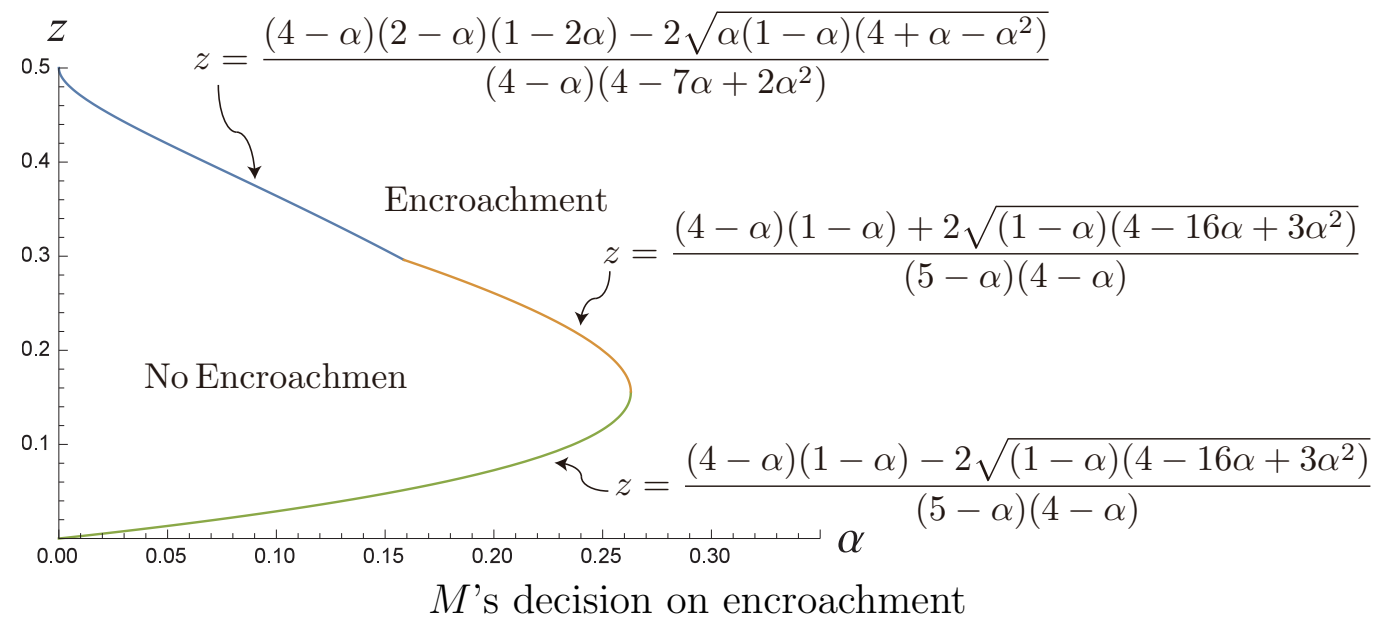

Figure 6: Encroachment decision (Encroachment-R\&D) 

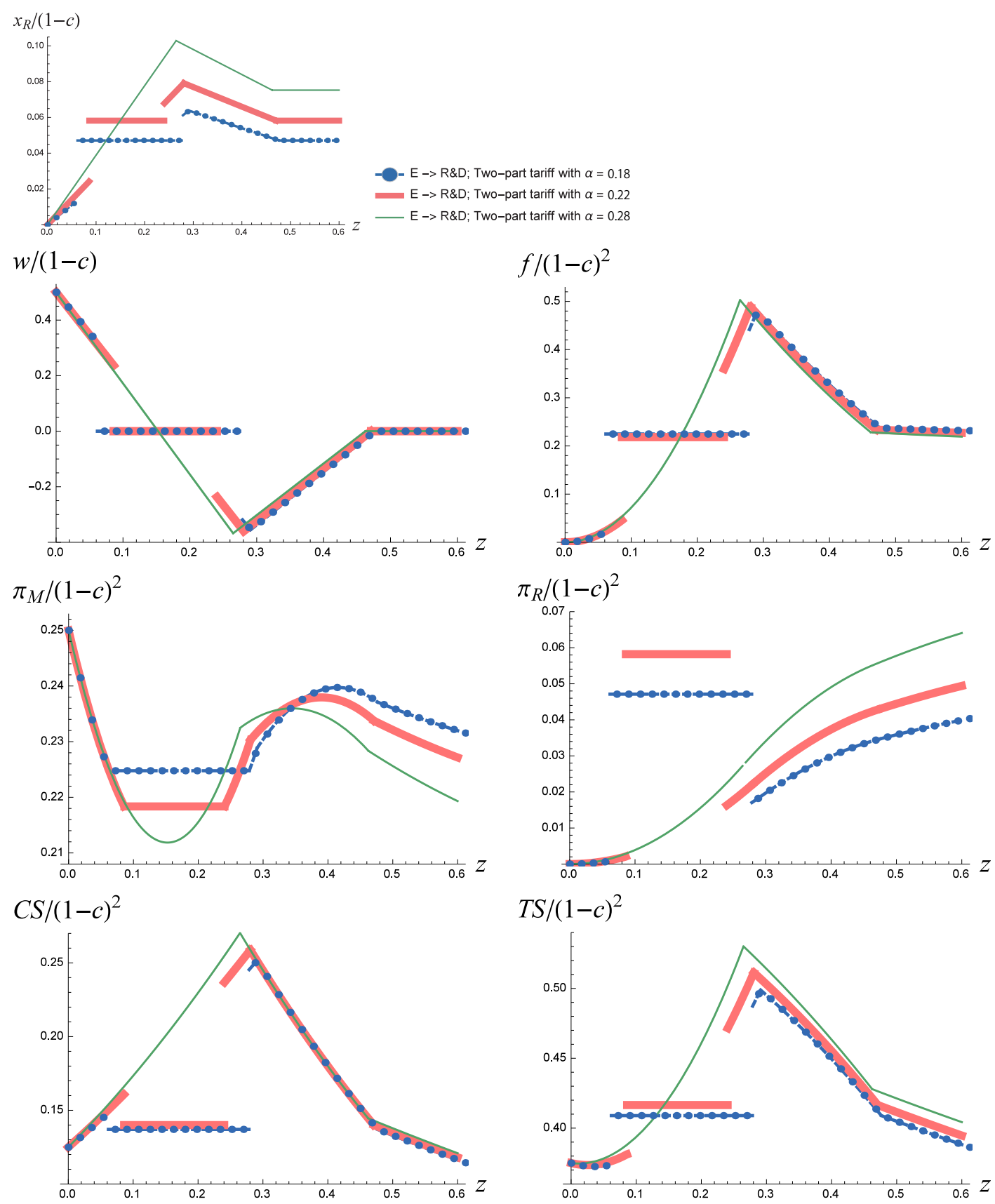

Figure 7: The outcomes under a two-part tariff (Encroachment-R\&D) 


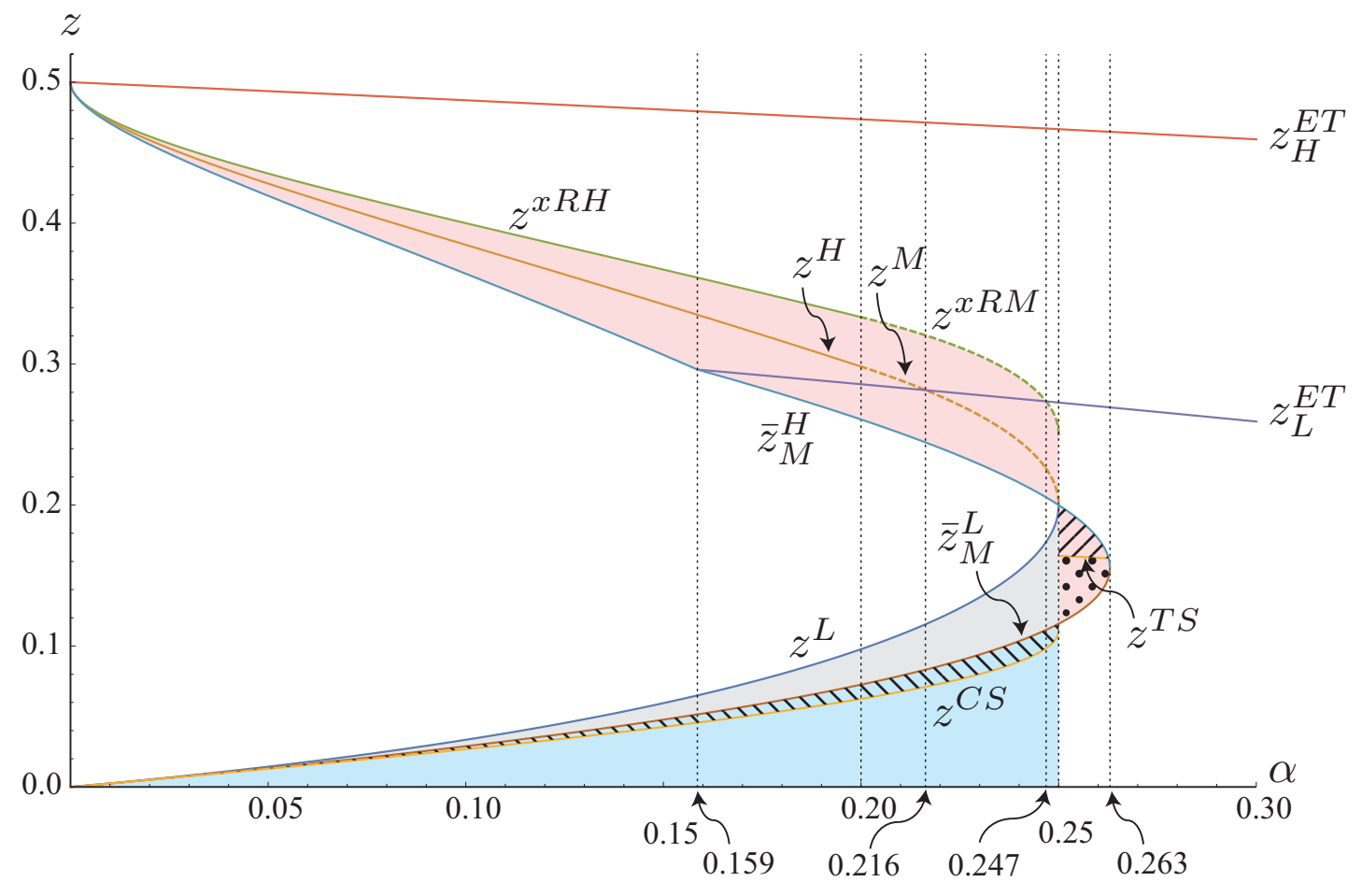

Figure 8: Commitment incentive and its effects 

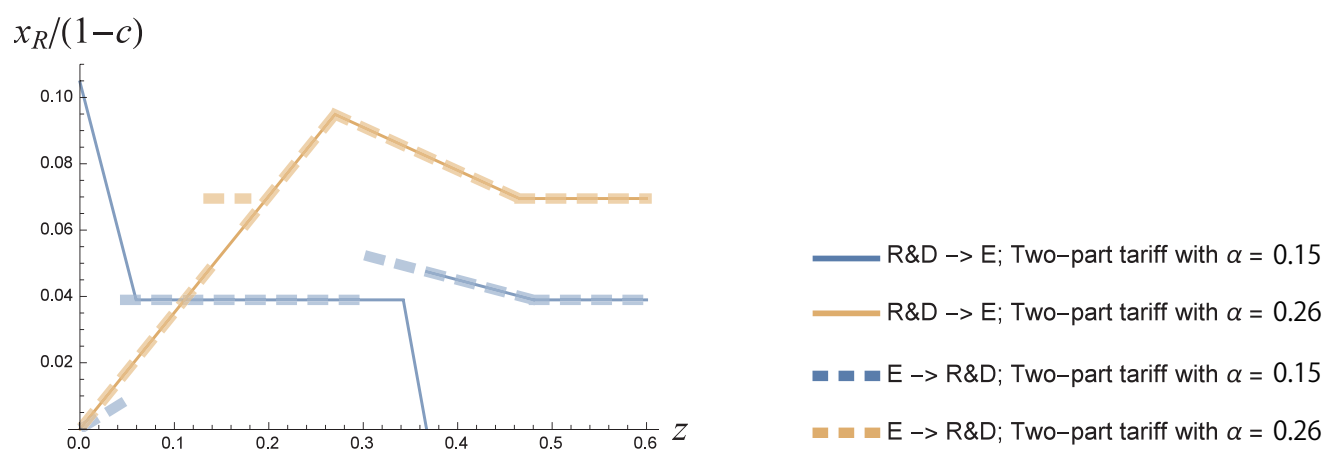

$w /(1-c)$

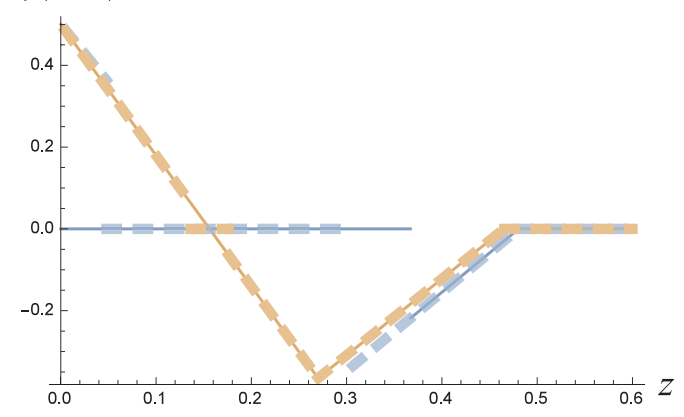

$f /(1-c)^{2}$
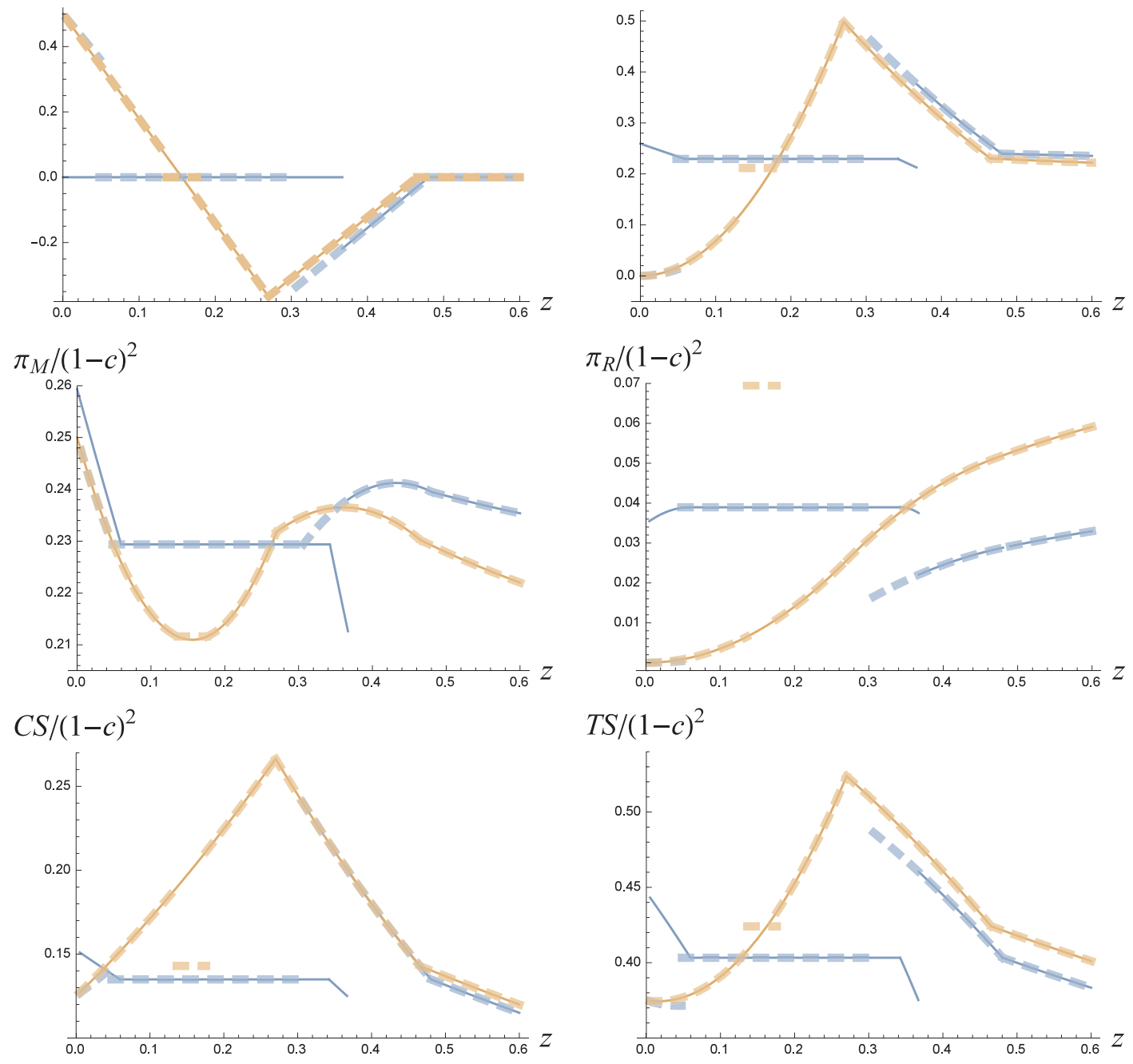

Figure 9: Comparison of the two scenarios under a two-part tariff 


\section{Online appendix on}

"Supplier encroachment and retailer effort"

by Noriaki Matsushima and Tomomichi Mizuno

\section{A Calculating equilibrium under linear contract}

In this appendix, we calculate the equilibrium. Here, we consider the case where the retailer $R$ and the manufacturer $M$ use a linear contract. The profits of $R$ and $M$ are $\pi_{R}=(1-$ $\left.q_{R}-q_{M}-\left(c-x_{R}\right)-w\right) q_{R}-x_{R}^{2}$ and $\pi_{M}=w q_{R}+\left(1-q_{R}-q_{M}-\left(c+c_{M}\right)\right) q_{M}$, respectively. Throughout this appendix, we assume $1>c+c_{M}, c>0$, and $c_{M}>0$. Note that if the first assumption is not satisfied, the direct channel output is always zero.

\section{A.1 Cost-reduction before encroachment}

First, we consider the case in which $R$ engages in cost reduction before $M$ 's encroachment decision.

\section{A.1.1 Stage 3 and 4: Cournot competition and wholesale pricing}

For the third and fourth stages, we have two subgames: that in which $M$ does not open its own direct channel (case $N$ ) and that in which it does open its own direct channel (case $E$ ).

Stages 3 and 4 in case $N$ (Section 3.1) When $M$ does not encroach (does not open its own direct channel), the maximization problem for $R$ in the fourth stage is $\max _{q_{R}}(1-$ $\left.q_{R}-\left(c-x_{R}\right)-w\right) q_{R}-x_{R}^{2}$, where $q_{M}=0$. Because $R$ is a unique channel, $q_{R}$ must be strictly positive (i.e., $\left.q_{R}>0\right)$. The first-order condition yields the output $q_{R}^{N}\left(w, x_{R}\right)=$ $\left(1-c-w+x_{R}\right) / 2$.

Substituting $q_{R}^{N}\left(w, x_{R}\right)$ into $\pi_{M}$, we have the maximization problem in the third stage $\max _{w} w q_{R}^{N}\left(w, x_{R}\right)$. Solving this problem, the wholesale price is $w^{N}\left(x_{R}\right)=\left(1-c+x_{R}\right) / 2$, leading to $q_{R}^{N}\left(x_{R}\right)=\left(1-c+x_{R}\right) / 4, \pi_{R}^{N}\left(x_{R}\right)$ in (5), and $\pi_{M}^{N}\left(x_{R}\right)$ in (6). 
Stage 4 in case $E$ (Section 3.2) First, we derive an interior solution by simply calculating the first-order conditions. Using the interior solution, we check the condition that the quantity supplied by each retail channel is positive.

In the fourth stage, the maximization problems for the retail channels of $R$ and $M$ are $\max _{q_{R}}\left(1-q_{R}-q_{M}-\left(c-x_{R}\right)-w\right) q_{R}-x_{R}^{2}$ and $\max _{q_{M}} w q_{R}+\left(1-q_{R}-q_{M}-\left(c+c_{M}\right)\right) q_{M}$, respectively. The first-order conditions lead to

$$
q_{R}\left(w, x_{R}\right)=\frac{1-c+c_{M}-2 w+2 x_{R}}{3}, \quad q_{M}\left(w, x_{R}\right)=\frac{1-c-2 c_{M}+w-x_{R}}{3} .
$$

By checking the signs of $q_{R}\left(w, x_{R}\right)$ and $q_{M}\left(w, x_{R}\right)$, we obtain the following relations: $q_{R}\left(w, x_{R}\right)>$ 0 and $q_{M}\left(w, x_{R}\right) \leq 0$ if $w \leq x_{R}-\left(1-c-2 c_{M}\right) \equiv \bar{w}_{L} ; q_{R}\left(w, x_{R}\right)>0$ and $q_{M}\left(w, x_{R}\right)>0$ if $\bar{w}_{L}<w<\left(1-c+c_{M}+2 x_{R}\right) / 2 \equiv \bar{w}_{H} ;$ and $q_{R}\left(w, x_{R}\right) \leq 0$ and $q_{M}\left(w, x_{R}\right)>0$ if $w \geq \bar{w}_{H}$. We check the corner solutions in the case of $w \leq \bar{w}_{L}$ and $w \geq \bar{w}_{H}$.

The outputs in the case of $w \leq \bar{w}_{L}$ are the same as those in case $N$. Then, the outputs are $q_{R}=q_{R}^{N}\left(w, x_{R}\right)$ and $q_{M}=0$. For the case of $w \geq \bar{w}_{H}$, we solve $\max _{q_{M}} w q_{R}+\left(1-q_{R}-\right.$ $\left.q_{M}-\left(c+c_{M}\right)\right) q_{M}$ s.t. $q_{R}=0$. Then, the outputs are $q_{R}=0$ and $q_{M}=\left(1-c-c_{M}\right) / 2$. Summarizing the above, we have the following outputs in the fourth stage.

$$
\left[q_{R}^{E}\left(w, x_{R}\right), q_{M}^{E}\left(w, x_{R}\right)\right]=\left\{\begin{array}{lr}
{\left[\frac{1-c+x_{R}-w}{2}, 0\right],} & \text { if } w \leq \bar{w}_{L}, \\
{\left[\frac{1-c+c_{M}+2 x_{R}-2 w}{3}, \frac{1-c-2 c_{M}-x_{R}+w}{3}\right]} & \text { if } \bar{w}_{L}<w<\bar{w}_{H}, \\
{\left[0, \frac{1-c-c_{M}}{2}\right]} & \text { if } \bar{w}_{H} \leq w .
\end{array}\right.
$$

Substituting these outputs into the profit equations, we have $\pi_{R}^{E}\left(w, x_{R}\right)=\left[q_{R}^{E}\left(w, x_{R}\right)\right]^{2}-$ $x_{R}^{2}$ and $\pi_{M}^{E}\left(w, x_{R}\right)=w q_{R}^{E}\left(w, x_{R}\right)+\left[q_{M}^{E}\left(w, x_{R}\right)\right]^{2}$.

Stage 3 in case $E$ We consider the three cases derived above: $w \leq \bar{w}_{L}, \bar{w}_{L}<w<\bar{w}_{H}$, and $w \geq \bar{w}_{H}$. Using the first-order condition in each case, we first calculate the candidate wholesale price.

First, we consider the profit of $R$ with $w \leq \bar{w}_{L}$. Solving the first-order condition $\partial \pi_{M}^{E}\left(w, x_{R}\right) / \partial w=0$, we have $w=\left(1-c+x_{R}\right) / 2 \equiv w_{+0}\left(x_{R}\right)$, where the subscript in- 
dicates that the quantities of $R$ and $M$ are strictly positive $(+)$ and zero $(0)$, respectively. Second, we consider the case of $\bar{w}_{L}<w<\bar{w}_{H}$. The first-order condition leads to $w=\left(5(1-c)-c_{M}+4 x_{R}\right) / 10 \equiv w_{++}\left(x_{R}\right)$, where the subscript means that the quantities of $R$ and $M$ are strictly positive $(+)$. Finally, we consider the case of $w \geq \bar{w}_{H}$. Because $q_{R}=0$ for any $w \in\left[\bar{w}_{H}, \infty\right)$, the profit of $M$ does not depend on $w$. Here, we assume that $M$ chooses $w=\bar{w}_{H} \equiv w_{0+}$, where the subscript indicates that the quantities of $R$ and $M$ are zero (0) and strictly positive $(+)$ respectively.

Next, we compare $\bar{w}_{L}, \bar{w}_{H}, w_{+0}$, and $w_{++}$. We define $x_{R}^{l} \equiv\left(5(1-c)-7 c_{M}\right) / 2$, which we obtain by solving $\bar{w}_{L}=w_{++}$for $x_{R}$, and $x_{R}^{h} \equiv 3(1-c)-4 c_{M}$, which we obtain by solving $\bar{w}_{L}=w_{+0}$.

Then, we have

$$
\begin{cases}\bar{w}_{L}<w_{++}<w_{+0}<\bar{w}_{H} & \text { if } x_{R}<x_{R}^{l} \\ w_{++} \leq \bar{w}_{L}<w_{+0}<\bar{w}_{H} & \text { if } x_{R}^{l} \leq x_{R}<x_{R}^{h} \\ w_{++}<w_{+0} \leq \bar{w}_{L}<\bar{w}_{H} & \text { if } x_{R}^{h} \leq x_{R}\end{cases}
$$

Moreover, we can show that $\pi_{R}^{E}\left(w, x_{R}\right)$ is continuous at $\bar{w}_{L}$ and $\bar{w}_{H}$. Because we must keep $w_{+0} \leq \bar{w}_{L}$ and $\bar{w}_{L}<w_{++}<\bar{w}_{H}$, the optimal wholesale price is as follows:

$$
w^{E}\left(x_{R}\right)= \begin{cases}\frac{5(1-c)-c_{M}+4 x_{R}}{10}\left(=w_{++}\right) & \text {if } x_{R}<x_{R}^{l} \\ x_{R}-\left(1-c-2 c_{M}\right)\left(=\bar{w}_{L}\right) & \text { if } x_{R}^{l} \leq x_{R}<x_{R}^{h} \\ \frac{1-c+x_{R}}{2}\left(=w_{+0}\right) & \text { if } x_{R}^{h} \leq x_{R} .\end{cases}
$$

Substituting $w^{E}\left(x_{R}\right)$ into the profit functions of $R$ and $M$, we have $\pi_{R}^{E}\left(x_{R}\right)$ in $(7)$ and $\pi_{M}^{E}\left(x_{R}\right)$ in (8). Similarly, substituting $w^{E}\left(x_{R}\right)$ into $q_{R}^{E}\left(w, x_{R}\right)$ and $q_{M}^{E}\left(w, x_{R}\right)$, we have $q_{R}^{E}\left(x_{R}\right)=q_{R}^{E}\left(w^{E}\left(x_{R}\right), x_{R}\right)$ and $q_{M}^{E}\left(x_{R}\right)=q_{M}^{E}\left(w^{E}\left(x_{R}\right), x_{R}\right)$.

\section{A.1.2 Stage 2}

Comparing $M$ 's profit under encroachment with that under no-encroachment, we show the condition in which $M$ encroaches. We have three cases, based on the level of investment $x_{R}$ : $x_{R} \in\left[0, x_{R}^{l}\right), x_{R} \in\left[x_{R}^{l}, x_{R}^{h}\right)$, and $x_{R} \in\left[x_{R}^{h}, \infty\right)$. We can easily show that in the second and third cases, $M$ does not have an incentive to encroach. That is, $\pi_{M}^{N}\left(x_{R}\right)>\pi_{R}^{E}\left(x_{R}\right)$. 
Here, we define $z \equiv c_{M} /(1-c)$. We consider the first case, $x_{R} \in\left[0, x_{R}^{l}\right)$. Calculating $\pi_{M}^{E}\left(x_{R}\right)-\pi_{R}^{N}\left(x_{R}\right)$, and substituting in $c_{M}=z(1-c)$, we have a quadratic function of $x_{R}$ with a positive coefficient of $x_{R}^{2}$. Solving $\pi_{M}^{E}\left(x_{R}\right)-\pi_{R}^{N}\left(x_{R}\right)=0$ for $x_{R}$, we have

$$
x_{R}=\left\{\begin{array}{l}
\frac{(1-c)(5-8 z-\sqrt{10}(1-z))}{3} \equiv \bar{x}_{R}, \\
\frac{(1-c)(5-8 z+\sqrt{10}(1-z))}{3} \equiv \bar{x}_{R}^{\prime} .
\end{array}\right.
$$

Comparing $\bar{x}_{R}, \bar{x}_{R}^{\prime}$, and $x_{R}^{l}$, we have $\bar{x}_{R}<x_{R}^{l}<\bar{x}_{R}^{\prime}$. Hence, we do not need to consider the threshold value $\bar{x}_{R}^{\prime}$. Then, $M$ opens its own direct channel if $x_{R}<\bar{x}_{R}$.

The profits of $R$ and $M$ are $\pi_{R}^{E}\left(x_{R}\right)$ and $\pi_{M}^{E}\left(x_{R}\right)$, respectively, if $x_{R}<\bar{x}_{R}$, and $\pi_{R}^{N}\left(x_{R}\right)$ and $\pi_{M}^{N}\left(x_{R}\right)$, respectively, if $x_{R} \geq \bar{x}_{R}\left(\pi_{R}\left(x_{R}\right)\right.$ in (10)).

\section{A.1.3 Stage 1}

From the result in the second stage, we have two cases: $x_{R}<\bar{x}_{R}$ and $x_{R} \geq \bar{x}_{R}$. First, we naively derive the "optimal" $x_{R}$ without taking into account the conditions in the two cases. Then, using the two "optimal" values of $x_{R}$, we check the true optimal $x_{R}$.

First, we consider the case of $x_{R}<\bar{x}_{R}$, in which the profit of $R$ is $\pi_{R}^{E}\left(x_{R}\right)$. Solving the first-order condition $\partial \pi_{R}^{E}\left(x_{R}\right) / \partial x_{R}=0$ for $x_{R}$, we have $x_{R}=4(1-c) z / 21 \equiv x_{R}^{E}$. Next, we consider the case of $x_{R} \geq \bar{x}_{R}$. The first-order condition $\partial \pi_{R}^{N}\left(x_{R}\right) / \partial x_{R}=0$ leads to $x_{R}=(1-c) / 15 \equiv x_{R}^{N}$.

In order to determine the true optimal investment level, we compare $x_{R}^{E}, x_{R}^{N}$, and $\bar{x}_{R}$. Then, we obtain the following:

$$
\begin{cases}x_{R}^{E}<\bar{x}_{R} \text { and } x_{R}^{N} \leq \bar{x}_{R} \quad \text { if } z \leq 0.339\left(\approx \frac{71-8 \sqrt{10}}{135}\right) \\ x_{R}^{E}<\bar{x}_{R} \text { and } \bar{x}_{R}<x_{R}^{N} \text { if } 0.339<z<0.340\left(\approx \frac{7(46-5 \sqrt{10})}{622}\right) \\ \bar{x}_{R} \leq x_{R}^{E} \text { and } \bar{x}_{R}<x_{R}^{N} \text { if } 0.340 \leq z\end{cases}
$$

where, after solving $x_{R}^{N}=\bar{x}_{R}$ and $x_{R}^{E}=\bar{x}_{R}$ for $z$, respectively, we obtain $z=0.339$ and $z=0.340$. This result means that the candidate optimal investment levels are $x_{R}^{E}$ and $\bar{x}_{R}$ if $z \leq 0.339, x_{R}^{E}$ and $x_{R}^{N}$ if $0.339<z<0.340$, and $x_{R}^{N}$ if $z \geq 0.340$. 
Comparing the profits of $R$ at the candidate levels, we show the equilibrium investment level. For the case of $z \leq 0.339$, we have $\pi_{R}^{E}\left(x_{R}^{E}\right)>\pi_{R}^{N}\left(\bar{x}_{R}\right)$ if $z<(847-2 \sqrt{70(8 \sqrt{10}-13)}-$ $224 \sqrt{10}) /(1327-280 \sqrt{10}) \approx 0.181$. Hence, $R$ chooses $x_{R}=x_{R}^{E}$ if $z<0.181$, and $x_{R}=\bar{x}_{R}$ if $0.181 \leq z \leq 0.339$. In the case of $0.339<z<0.340$, we have $\pi_{R}^{E}\left(x_{R}^{E}\right)<\pi_{R}^{N}\left(x_{R}^{N}\right)$. Hence, $R$ chooses $x_{R}=x_{R}^{N}$. In the case of $z \geq 0.340$, because we have $\pi_{R}^{E}\left(\bar{x}_{R}\right)<\pi_{R}^{N}\left(x_{R}^{N}\right), R$ chooses $x_{R}^{N}$.

Summarizing the above results, we obtain the equilibrium investment level described in Proposition 1:

$$
x_{R}^{*}= \begin{cases}x_{R}^{E}=\frac{4(1-c) z}{21} & \text { if } z \leq 0.181, \\ \bar{x}_{R}=\frac{(1-c)\{(5-\sqrt{10})-(8-\sqrt{10}) z\}}{3} & \text { if } 0.181<z \leq 0.339, \\ x_{R}^{N}=\frac{1-c}{15} & \text { if } z>0.339 .\end{cases}
$$

Finally, we calculate the equilibrium outcomes by substituting $x_{R}=x_{R}^{*}$ into $q_{R}^{N}\left(x_{R}\right)$, $w^{N}\left(x_{R}\right), \pi_{R}^{N}\left(x_{R}\right), \pi_{M}^{N}\left(x_{R}\right), q_{R}^{E}\left(x_{R}\right), q_{M}^{E}\left(x_{R}\right), w^{E}\left(x_{R}\right), \pi_{R}^{E}\left(x_{R}\right)$, and $\pi_{M}^{E}\left(x_{R}\right)$. Here, we divide the outputs and the wholesale price by $1-c$, and divide the profits by $(1-c)^{2}$. Note that $x_{R}^{E}<x_{R}^{l}$ for $z \leq 0.181$.

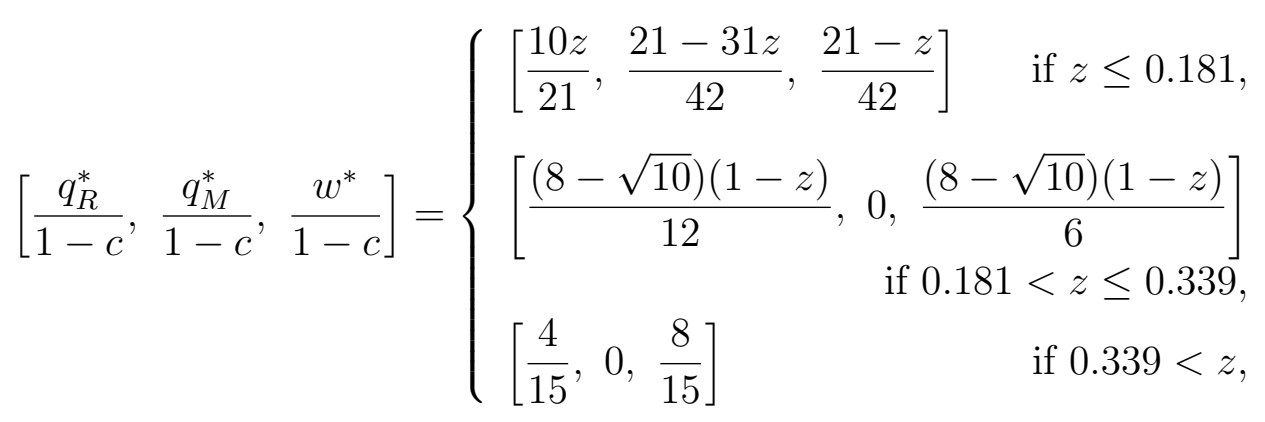




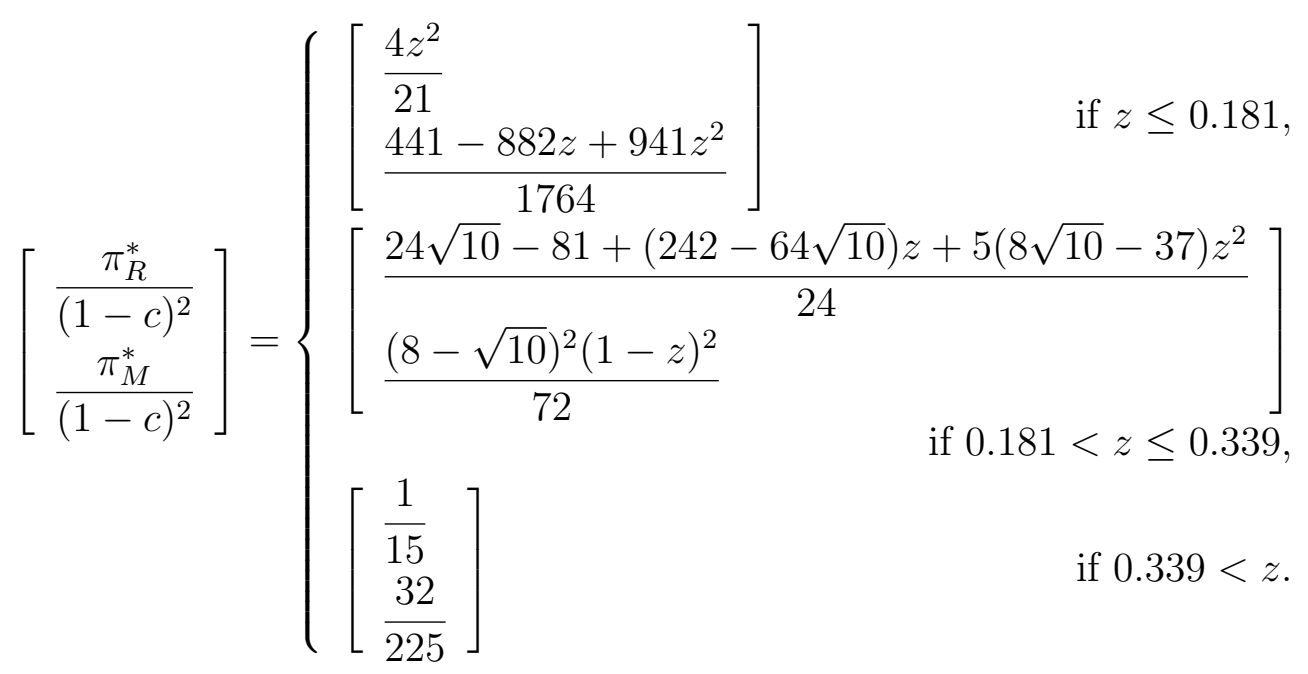

Substituting the above results into $C S, P S$, and $T S$, we have the equilibrium surpluses $C S^{*}$, $P S^{*}$, and $T S^{*}$, respectively. Note that after dividing these functions by $(1-c)^{2}$, they include only the parameter $z$.

Because these outcomes contain only parameter $z$ after dividing by $1-c$ or $(1-c)^{2}$, we can depict them as shown in Figure 1. Moreover, differentiating these outcomes with respect to $z$, we complete the proof of Proposition 1.

\section{A.2 Cost-reduction after encroachment}

For the third and the fourth stages, we have the same outcomes as in the case of costreduction before encroachment. Hence, we start with the second stage, where $R$ chooses an investment level.

\section{A.2.1 Stage 2}

In this stage, $R$ chooses an investment level. Following the decision in the first stage, we have two subgames: that in which $M$ does not open its own direct channel (case $N$ ), and that in which it does open its own direct channel (case $E$ ).

Stage 2 (case $N$ ) First, we consider the case without encroachment. The profit of $R$ is $\pi_{R}^{N}\left(x_{R}\right)$ in $(10)$, and the first-order condition leads to $x_{R}=(1-c) / 15\left(=x_{R}^{N}\right)$. Then, the profits of $R$ and $M$ are $\pi_{R}^{N}\left(x_{R}^{N}\right)$ and $\pi_{M}^{N}\left(x_{R}^{N}\right)$, respectively. 
Stage 2 (case $E$ ) Based on the investment levels, we have three cases: $x_{R}<x_{R}^{l}, x_{R}^{l} \leq$ $x_{R}<x_{R}^{h}$, and $x_{R}^{h} \leq x_{R}$. First, we naively derive $x_{R}$ that satisfies the first-order conditions in each of the three cases. These $x_{R}$ are the candidates for the optimal investment level.

For $x_{R}<x_{R}^{l}$, the first-order condition leads to $x_{R}=4(1-c) z / 21\left(=x_{R}^{E}\right)$. For $x_{R}^{l} \leq x_{R}<$ $x_{R}^{h}$, the first-order condition yields $x_{R}=0$. For $x_{R}^{h} \leq x_{R}$, solving the first-order condition, we have $x_{R}=(1-c) / 15\left(=x_{R}^{N}\right)$.

Comparing $x_{R}^{E}, x_{R}^{N}, x_{R}^{l}, x_{R}^{h}$, and 0 , we have the following inequalities.

$$
\begin{cases}x_{R}^{E} \leq x_{R}^{l}, \quad 0<x_{R}^{l}, \quad \text { and } x_{R}^{N}<x_{R}^{h} & \text { if } 0<z \leq \frac{21}{31}(\approx 0.677), \\ x_{R}^{l}<x_{R}^{E}, \quad 0 \leq x_{R}^{l}, \quad \text { and } x_{R}^{N}<x_{R}^{h} & \text { if } 0.677<z \leq \frac{5}{7}(\approx 0.714), \\ x_{R}^{l}<x_{R}^{E}, \quad x_{R}^{l}<0<x_{R}^{h}, \quad \text { and } x_{R}^{N} \leq x_{R}^{h} & \text { if } 0.714<z \leq \frac{11}{15}(\approx 0.733), \\ x_{R}^{l}<x_{R}^{E}, \quad x_{R}^{l}<0 \leq x_{R}^{h}, \quad \text { and } x_{R}^{h}<x_{R}^{N} & \text { if } 0.733<z \leq \frac{3}{4}(=0.75), \\ x_{R}^{l}<x_{R}^{E}, \quad x_{R}^{h}<0, \quad \text { and } x_{R}^{h}<x_{R}^{N} & \text { if } 0.75<z .\end{cases}
$$

Note that we find that the profit function of $R, \pi_{R}^{E}\left(x_{R}\right)$, is continuous with respect to $x_{R}$ by substituting $x_{R}=x_{R}^{l}$ or $x_{R}=x_{R}^{h}$ into $\pi_{R}^{E}\left(x_{R}\right)$. Moreover, $\pi_{R}^{E}\left(x_{R}\right)$ is locally concave over $x_{R}$ for each of the three cases. For $z \leq 0.677, x_{R}=x_{R}^{E}$ is the local optimal value on the range $\left[0, x_{R}^{l}\right)$, and $\pi_{R}^{E}\left(x_{R}\right)$ decreases in $x_{R}$ on $\left[x_{R}^{l}, \infty\right)$. Hence, $x_{R}=x_{R}^{E}$ is the global optimal value. For $0.677<z \leq 0.714, \pi_{R}^{E}\left(x_{R}\right)$ increases in $x_{R}$ on $\left[0, x_{R}^{l}\right)$, and decreases in $x_{R}$ on $\left[x_{R}^{l}, \infty\right)$. Hence, $x_{R}=x_{R}^{l}$ is the global optimal value. For $0.714<z \leq 0.733, \pi_{R}^{E}\left(x_{R}\right)$ monotonically decreases in $x_{R}$ for $x_{R} \geq 0\left(>x_{R}^{l}\right)$. Hence, $x_{R}=0$ is the global optimal value. For $z>0.75$, $\pi_{R}^{E}\left(x_{R}\right)$ is maximized at $x_{R}=x_{R}^{N}$ for $x_{R} \geq 0\left(\geq x_{R}^{h}\right)$.

The remaining case is $0.733<z \leq 0.75$. In this case, we have two candidates for the optimal investment level: $x_{R}=0$ for $\left(x_{R}^{l}<\right) 0 \leq x_{R}<x_{R}^{h}$, and $x_{R}=x_{R}^{N}$ for $x_{R} \geq x_{R}^{h}$. Substituting these into $\pi_{R}^{E}\left(x_{R}\right)$, we have $\pi_{R}^{E}(0) \geq \pi_{R}^{E}\left(x_{R}^{N}\right)$ if $0.733<z \leq(15-\sqrt{15}) / 15(\approx$ $0.742)$, and $\pi_{R}^{E}(0)<\pi_{R}^{E}\left(x_{R}^{N}\right)$ if $0.742<z \leq 0.75$, respectively. 
Summarizing the above results, we have the following outcome in the second stage.

$$
x_{R}^{E 2 n d}= \begin{cases}\frac{4(1-c) z}{21}\left(=x_{R}^{E}\right) & \text { if } z \leq 0.677 \\ \frac{(1-c)(5-7 z)}{2}\left(=x_{R}^{l}\right) & \text { if } 0.677<z \leq 0.714 \\ 0 & \text { if } 0.714<z \leq 0.742, \\ \frac{1-c}{15}\left(=x_{R}^{N}\right) & \text { if } 0.742<z .\end{cases}
$$

Substituting $x_{R}=x_{R}^{E 2 n d}$ into the profits of $R$ and $M$, we have the following:

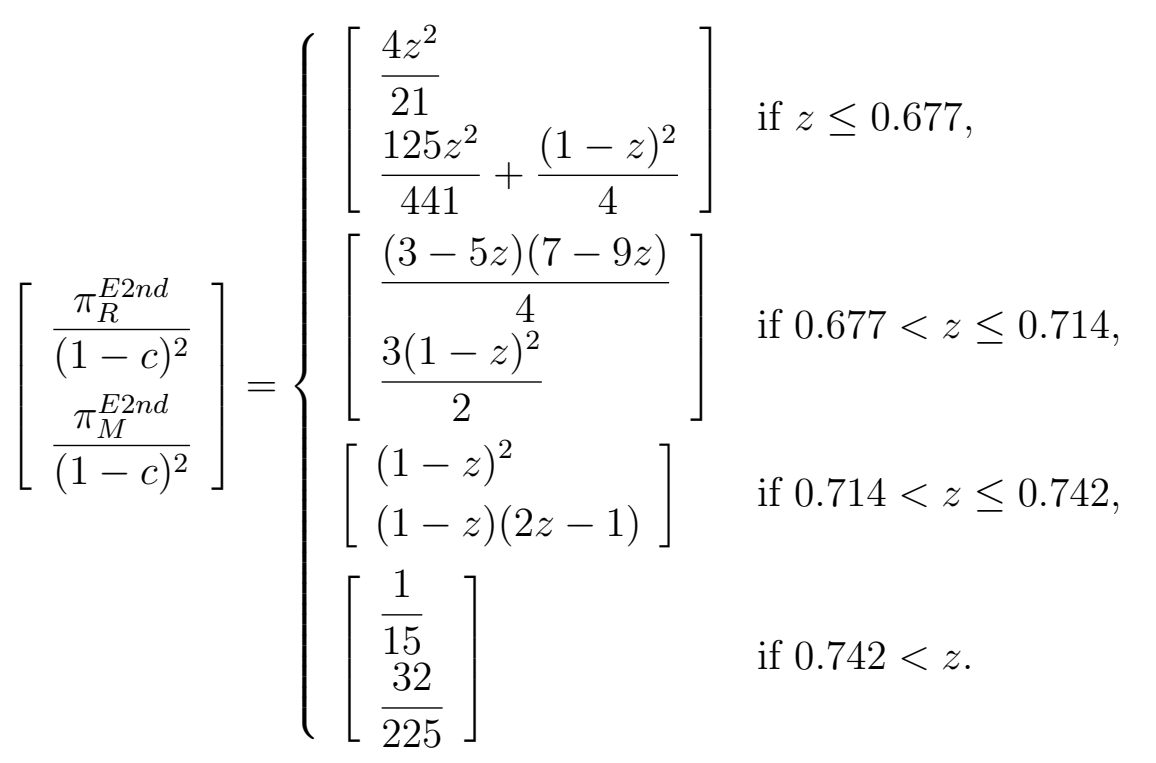

\section{A.2.2 Stage 1}

We derive Proposition 2 by comparing $\pi_{M}^{N}\left(x_{R}^{N}\right)$ and $\pi_{M}^{E 2 n d}$. For $z \leq 0.677, \pi_{M}^{E 2 n d}>\pi_{M}^{N}\left(x_{R}^{N}\right)$ if $z<7(315-2 \sqrt{1987}) / 4705(\approx 0.336)$ or $7(315+2 \sqrt{1987}) / 4705(\approx 0.601)<z \leq 0.677$. For $0.677<z \leq 0.714, \pi_{M}^{E 2 n d}>\pi_{M}^{N}\left(x_{R}^{N}\right)$ if $0.677<z<(45-8 \sqrt{3}) / 45(\approx 0.692)$. For $0.714<z, \pi_{M}^{E 2 n d}<\pi_{M}^{N}\left(x_{R}^{N}\right)$. Hence, $M$ encroaches when the following condition is satisfied (as in Proposition 2):

$$
z<0.336 \text { or } 0.601<z<0.692 \text {. }
$$

As in the case with investment before encroachment, using the threshold values $z=$ 
0.336, 0.601, 0.677, and 0.692, we can calculate the equilibrium outcomes, as follows:

$$
\begin{aligned}
& {\left[\begin{array}{ll}
\frac{q_{R}^{* *}}{1-c}, \frac{q_{M}^{* *}}{1-c} \\
\frac{w^{* *}}{1-c}, \frac{x_{R}^{* *}}{1-c}
\end{array}\right]= \begin{cases}{\left[\begin{array}{ll}
\frac{10 z}{21}, & \frac{21-31 z}{42} \\
\frac{21-z}{42}, \frac{4 z}{21}
\end{array}\right]} & \text { if } z<0.336 \text { or } 0.601<z<0.677, \\
{\left[\begin{array}{ll}
\frac{4}{15}, 0 \\
\frac{8}{15}, \frac{1}{15}
\end{array}\right]} & \text { if } 0.336 \leq z \leq 0.601 \quad \text { or } \quad 0.692 \leq z, \\
{\left[\begin{array}{ll}
1-z, & 0 \\
\frac{3(1-z)}{2}, & \frac{5-7 z}{2}
\end{array}\right] \quad \text { if } 0.677 \leq z<0.692,}\end{cases} }
\end{aligned}
$$

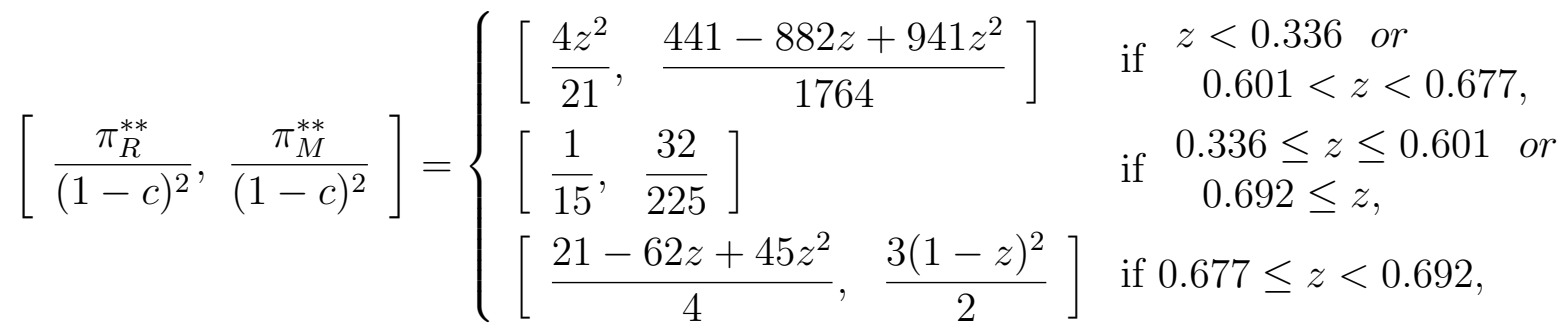

Substituting the above results into $C S, P S$, and $T S$, we have the equilibrium surpluses $C S^{* *}, P S^{* *}$, and $T S^{* *}$, respectively. Note that after dividing these functions by $(1-c)^{2}$, they include only parameter $z$.

Using the above outcomes, we derive Figure 2. Moreover, differentiating these outcomes with respect to $z$, we complete the proof of Proposition 2.

\section{B Calculating the equilibrium under a two-part tariff}

Here, we calculate the equilibrium for a two-part tariff. The profits of $R$ and $M$ are $\pi_{R}=$ $\left(p-\left(c-x_{R}\right)-w\right) q_{R}-x_{R}^{2}-f$ and $\pi_{M}=w q_{R}+\left(p-\left(c+c_{M}\right)\right) q_{M}+f$, respectively.

\section{B.1 Cost-reduction before encroachment}

\section{B.1.1 No encroachment subgame (Case NT)}

First, we consider the case without encroachment. In the fourth stage, $M$ 's output is zero and $R$ chooses $q_{R}^{N}\left(x_{R}, w\right)=\left(1-c+x_{R}-w\right) / 2=\arg \max _{q_{R}} \pi_{R}$. Then, the resulting profits 
of $R$ and $M$ are $\pi_{R}^{N}\left(x_{R}, w\right)=\left(q_{R}^{N}\left(x_{R}, w\right)\right)^{2}-f-x_{R}^{2}$ and $\pi_{M}^{N}\left(x_{R}, w\right)=w q_{R}^{N}\left(x_{R}, w\right)+f$, respectively.

In the third stage, $M$ and $R$ negotiate a two-part tariff. Then, the bargaining outcome is $\left(w^{N}, f^{N}\left(x_{R}\right)\right)=\left(0,(1-\alpha)\left(1-c+x_{R}\right)^{2} / 4\right)=\arg \max \alpha \log \left[\pi_{R}^{N}\left(x_{R}, w\right)-\left(-x_{R}^{2}\right)\right]+(1-$ $\alpha) \log \left[\pi_{M}^{N}\left(x_{R}, w\right)\right]$. From the third and fourth stage outcomes, we obtain the profits of $R$ and $M$ as (16) and (17), respectively:

$$
\pi_{R}^{N T}\left(x_{R}\right)=\frac{\alpha\left(1-c+x_{R}\right)^{2}}{4}-x_{R}^{2}, \quad \pi_{M}^{N T}\left(x_{R}\right)=\frac{(1-\alpha)\left(1-c+x_{R}\right)^{2}}{4} .
$$

\section{B.1.2 Encroachment subgame (Case ET)}

We consider the subgame under encroachment. In the fourth stage, the first-order conditions lead to

$$
\begin{aligned}
& q_{R}^{E}\left(x_{R}, w\right)= \begin{cases}\frac{(1-c)(1+z)+2 x_{R}-2 w}{3} & \text { if } w>x_{R}-(1-c)(1-2 z) \equiv \bar{w}\left(x_{R}\right), \\
\frac{1-c+x_{R}-w}{2} & \text { if } w \leq \bar{w}\left(x_{R}\right),\end{cases} \\
& q_{M}^{E}\left(x_{R}, w\right)= \begin{cases}\frac{(1-c)(1-2 z)-x_{R}+w}{3} & \text { if } w>\bar{w}\left(x_{R}\right), \\
0 & \text { if } w \leq \bar{w}\left(x_{R}\right) .\end{cases}
\end{aligned}
$$

Substituting these into the profits of $R$ and $M$, we have $\pi_{R}^{E}=\left(q_{R}^{E}\left(x_{R}, w\right)\right)^{2}-f-x_{R}^{2}$ and $\pi_{M}^{E}\left(x_{R}, w\right)=\left(q_{M}^{E}\left(x_{R}, w\right)\right)^{2}+w q_{R}^{E}\left(x_{R}, w\right)+f$, respectively.

In the third stage, $R$ and $M$ negotiate a two-part tariff. The outside option for $R$ is the same as that in the case without encroachment: $-x_{R}^{2}$. On the other hand, even if the negotiation breaks down, $M$ can sell its product through its own direct channel. Hence, the outside profit is $\pi_{M}^{O}=(1-c)^{2}(1-z)^{2} / 4=\max _{q_{M}} \pi_{M}$ s.t. $q_{R}=0$. Then, the bargaining problem is

$$
\max _{w, f} \alpha \log \left[\pi_{R}^{E}\left(x_{R}, w\right)-\left(-x_{R}^{2}\right)\right]+(1-\alpha) \log \left[\pi_{M}^{E}\left(x_{R}, w\right)-\pi_{M}^{O}\right]
$$

Because the outside profit $\pi_{M}^{O}$ does not depend on $w$ and $f$, we can solve this maximization problem as follows. First, we maximize the net joint profit, $\pi_{R}^{E}\left(x_{R}, w\right)-\left(-x_{R}^{2}\right)+\pi_{M}^{E}\left(x_{R}, w\right)-$ $\pi_{M}^{O}$, with respect to $w$. Second, we divide the maximized net joint profit by $f$. Specifically, we 
first obtain $w^{E T}\left(x_{R}\right)=\arg \max _{w} \pi_{R}^{E}\left(x_{R}, w\right)-\left(-x_{R}^{2}\right)+\pi_{M}^{E}\left(x_{R}, w\right)-\pi_{M}^{O}=\arg \max _{w} \Pi\left(x_{R}, w\right)$, where $\Pi\left(x_{R}, w\right) \equiv \pi_{R}^{E}\left(x_{R}, w\right)+\pi_{M}^{E}\left(x_{R}, w\right)$, which is the actual joint profit. Second, substituting $w^{E T}\left(x_{R}\right)$ into the profits of $R$ and $M$, we solve the following equation for $f$ : $\pi_{R}^{E}\left(x_{R}, w^{E T}\right)-\left(-x_{R}^{2}\right)=\alpha\left[\pi_{R}^{E}\left(x_{R}, w^{E T}\right)-\left(-x_{R}^{2}\right)+\pi_{M}^{E}\left(x_{R}, w^{E T}\right)-\pi_{M}^{O}\right]$. In this way, we obtain the fixed fee $f=f^{E T}\left(x_{R}\right)$.

Here, we derive $w^{E T}$ and $f^{E T}$. Because $q_{M}^{E}\left(x_{R}, w\right)$ and $q_{R}^{E}\left(x_{R}, w\right)$ are continuous in $w$, $\Pi\left(x_{R}, w\right)$ is also continuous in $w$. First, we calculate candidates for $w^{E T}$. Using $q_{M}^{E}\left(x_{R}, w\right)$ and $q_{R}^{E}\left(x_{R}, w\right)$ for the case with $w>\bar{w}\left(x_{R}\right)$, we solve $\partial \Pi\left(x_{R}, w\right) / \partial w=0$, leading to $w=$ $\left((1-c)(1-5 z)-4 x_{R}\right) / 2 \equiv w^{E T+}\left(x_{R}\right)$. In addition, using $q_{R}^{E}\left(x_{R}, w\right)$ and $q_{M}^{E}\left(x_{R}, w\right)$ for the case $w \leq \bar{w}\left(x_{R}\right)$, we solve $\partial \Pi\left(x_{R}, w\right) / \partial w=0$, leading to $w=0$. Comparing $w^{E T+}\left(x_{R}\right)$ and 0 with $\bar{w}\left(x_{R}\right)$, we have $\bar{w}\left(x_{R}\right)<0$ and $\bar{w}\left(x_{R}\right)<w^{E T+}\left(x_{R}\right)$ if $x_{R}<(1-c)(1-3 z) / 2 \equiv K_{L}$, $\bar{w}\left(x_{R}\right)<0$ and $w^{E T+}\left(x_{R}\right) \leq \bar{w}\left(x_{R}\right)$ if $K_{L} \leq x_{R}<(1-c)(1-2 z) \equiv K_{H}$, and $0 \leq \bar{w}\left(x_{R}\right)$ and $w^{E T+}\left(x_{R}\right)<\bar{w}\left(x_{R}\right)$ if $K_{H} \leq x_{R}$. From the three cases and the continuity of $\Pi\left(x_{R}, w\right)$, we obtain $w^{E T}\left(x_{R}\right)$, as follows:

$$
w^{E T}\left(x_{R}\right)= \begin{cases}\frac{(1-c)(1-5 z)-4 x_{R}}{2}\left(=w^{E T+}\left(x_{R}\right)\right) & \text { if } x_{R}<K_{L}, \\ -(1-c)(1-2 z)+x_{R}\left(=\bar{w}\left(x_{R}\right)\right) & \text { if } K_{L} \leq x_{R}<K_{H}, \\ 0 & \text { if } K_{H} \leq x_{R}\end{cases}
$$

Second, we calculate $f^{E T}$. Substituting $w^{E T}$ into $\pi_{R}^{E}\left(x_{R}, w^{E T}\right)-\left(-x_{R}^{2}\right)=\alpha\left[\pi_{R}^{E}\left(x_{R}, w^{E T}\right)-\right.$ $\left.\left(-x_{R}^{2}\right)+\pi_{M}^{E}\left(x_{R}, w^{E T}\right)-\pi_{M}^{O}\right]$ and solving for $f$, we have

$$
f^{E T}\left(x_{R}\right)= \begin{cases}(4-\alpha)\left((1-c) z+x_{R}\right)^{2} & \text { if } x_{R}<K_{L}, \\ \frac{(1-c)(1-z)\left((1-c)(4-\alpha-(4-5 \alpha) z)+4 \alpha x_{R}\right)}{4} & \text { if } K_{L} \leq x_{R}<K_{H}, \\ \frac{(1-\alpha)\left(1-c+x_{R}\right)^{2}+\alpha(1-c)^{2}(1-z)^{2}}{4} & \text { if } K_{H} \leq x_{R} .\end{cases}
$$

Substituting $w^{E T}\left(x_{R}\right)$ and $f^{E T}\left(x_{R}\right)$ into $\pi_{R}^{E}\left(x_{R}, w\right)$ and $\pi_{M}^{E}\left(x_{R}, w\right)$, we obtain the profits of $R$ and $M$ as (18) and (19), respectively:

$$
\pi_{R}^{E T}\left(x_{R}\right)= \begin{cases}\alpha\left((1-c) z+x_{R}\right)^{2}-x_{R}^{2} & \text { if } x_{R}<K_{L}, \\ \frac{(1-c)(1-z)\left(-(1-c)(1-5 z)+4 x_{R}\right)}{4}-x_{R}^{2} & \text { if } K_{L} \leq x_{R}<K_{H}, \\ \frac{\alpha\left((1-c) z+x_{R}\right)\left((1-c)(2-z)+x_{R}\right)}{4}-x_{R}^{2} & \text { if } K_{H} \leq x_{R},\end{cases}
$$




$$
\pi_{M}^{E T}\left(x_{R}\right)= \begin{cases}\frac{(1-c)^{2}(1-z)^{2}+4(1-\alpha)\left((1-c) z+x_{R}\right)^{2}}{4} & \text { if } x_{R}<K_{L}, \\ \frac{(1-c)(1-z)\left((1-c)(\alpha+(4-5 \alpha) z)+4(1-\alpha) x_{R}\right)}{4} & \text { if } K_{L} \leq x_{R}<K_{H}, \\ \frac{(1-\alpha)\left(1-c+x_{R}\right)^{2}+\alpha(1-c)^{2}(1-z)^{2}}{4} & \text { if } K_{H} \leq x_{R} .\end{cases}
$$

\section{B.1.3 Stage 2}

In the second stage, $M$ decides whether to open its own direct channel. We consider the three cases described earlier: (i) $x_{R}<K_{L}$; (ii) $K_{L} \leq x_{R}<K_{H}$; and (iii) $K_{H} \leq x_{R}$.

Case (i) For $x_{R}<K_{L}$, we consider the sign of $\pi_{M}^{E T}\left(x_{R}\right)-\pi_{M}^{N T}\left(x_{R}\right)$. This difference is a quadratic function of $x_{R}$, the coefficient of $x_{R}^{2}$ takes a positive value, and the discriminant of this difference takes a negative value if $\alpha>1 / 4$, implying that $M$ opens its own direct channel for any $x_{R}<K_{L}$ if $\alpha>1 / 4$.

On the other hand, if $\alpha \leq 1 / 4$, there are one or two real roots that satisfy $\pi_{M}^{E T}\left(x_{R}\right)-$ $\pi_{M}^{N T}\left(x_{R}\right)=0$. We denote the roots by $\bar{x}_{R}^{L}$ and $\bar{x}_{R}^{M}$.

$$
\begin{aligned}
& \bar{x}_{R}^{L} \equiv \frac{(1-c)\{(1-\alpha)(1-4 z)-(1-z) J\}}{3(1-\alpha)}, \\
& \bar{x}_{R}^{M} \equiv \frac{(1-c)\{(1-\alpha)(1-4 z)+(1-z) J\}}{3(1-\alpha)},
\end{aligned}
$$

where $J=\sqrt{(1-4 \alpha)(1-\alpha)}$ defined in $(20)$ and $x_{R}^{L} \leq x_{R}^{M}$.

We can show that $\bar{x}_{R}^{L}<K_{L}$ for any $\alpha \leq 1 / 4$. Comparing $\bar{x}_{R}^{M}$ with $K_{L}$, we have $\bar{x}_{R}^{M}<K_{L}$ if $1 / 5<\alpha \leq 1 / 4$. Hence, if $\alpha \leq 1 / 5$, the condition for opening the direct channel is $x_{R}<\bar{x}_{R}^{L}$, given $1 / 5<\alpha \leq 1 / 4$; that is, $x_{R}<\bar{x}_{R}^{L}$ or $\bar{x}_{R}^{M}<x_{R}<K_{L}$.

Case (ii) For $K_{L} \leq x_{R}<K_{H}$, we consider the sign of $\pi_{M}^{E T}\left(x_{R}\right)-\pi_{M}^{N T}\left(x_{R}\right)$, as in Case (i). The coefficient of $x_{R}^{2}$ is negative, the discriminant takes a positive value, and there are two roots of the equation $\pi_{M}^{E T}\left(x_{R}\right)-\pi_{M}^{N T}\left(x_{R}\right)=0$, given by

$$
\begin{aligned}
& \bar{x}_{R}^{h}=\frac{(1-c)\{(1-\alpha)(1-2 z)+(1-z) \sqrt{\alpha(1-\alpha)}\}}{1-\alpha}, \\
& \bar{x}_{R}^{H}=\frac{(1-c)\{(1-\alpha)(1-2 z)-(1-z) \sqrt{\alpha(1-\alpha)}\}}{1-\alpha} .
\end{aligned}
$$


Comparing $\bar{x}_{R}^{h}$ and $\bar{x}_{R}^{H}$ with $K_{L}$ and $K_{H}$, we have $K_{L} \leq \bar{x}_{R}^{H}<K_{H}<\bar{x}_{R}^{h}$ if $\alpha \leq 1 / 5$, and $\bar{x}_{R}^{H}<K_{L}<K_{H}<\bar{x}_{R}^{h}$ if $\alpha>1 / 5$. Hence, the condition in which $M$ opens its own direct channel is (1) $\bar{x}_{R}^{H}<x_{R}<K_{H}$ and $\alpha \leq 1 / 5$, or (2) $K_{L} \leq x_{R}<K_{H}$ and $\alpha>1 / 5$.

Case (iii) For any $x_{R} \geq K_{H}, \pi_{M}^{E T}\left(x_{R}\right)-\pi_{M}^{N T}\left(x_{R}\right)>0$, implying that $M$ opens its own direct channel.

Summarizing the above arguments, we obtain the condition that $M$ opens its own direct channel, as in (20).

$$
\left\{\begin{array}{lll}
x_{R}<\bar{x}_{R}^{L} \text { or } & x_{R}>\bar{x}_{R}^{H} & \text { if } \alpha \leq 1 / 5 \\
x_{R}<\bar{x}_{R}^{L} \text { or } & x_{R}>\bar{x}_{R}^{M} & \text { if } 1 / 5<\alpha \leq 1 / 4 \\
\text { for any } x_{R} & & \text { if } \alpha>1 / 4
\end{array}\right.
$$

\section{B.1.4 Stage 1}

We consider the investment decision by $R$. From the result in the second stage, the encroachment decision depends on the investment level $x_{R}$ and the $R$ 's bargaining power $\alpha$. We consider the three cases derived earlier: (I) $\alpha \leq 1 / 5$, (II) $1 / 5<\alpha \leq 1 / 4$, (III) $\alpha>1 / 4$.

Case (I): $\alpha \leq 1 / 5 \quad$ From the result in the second stage, $M$ encroaches if $x_{R}<\bar{x}_{R}^{L}$ or $x_{R}>\bar{x}_{R}^{H}$.

First, we consider the investment level under encroachment in the following intervals for $x_{R}: x_{R}<\bar{x}_{R}^{L}$ and $x_{R}>\bar{x}_{R}^{H}$. From the result in the third stage, we have to consider a different two-part tariff for each interval of $x_{R}:\left[0, \bar{x}_{R}^{L}\right),\left(\bar{x}_{R}^{H}, K_{H}\right)$, and $\left[K_{H}, \infty\right)$. Solving $\partial \pi_{R}^{E T}\left(x_{R}\right) / \partial x_{R}=0$ for each interval, we have

$$
x_{R}=\alpha c_{M} /(1-\alpha) \equiv x_{R}^{E T L}
$$

for the case of $0 \leq x_{R}<\bar{x}_{R}^{L}$,

$$
x_{R}=\alpha\left(1-c-c_{M}\right) / 2 \equiv x_{R}^{E T M}
$$

for the case of $x_{R} \in\left(\bar{x}_{R}^{H}, K_{H}\right)$, and

$$
x_{R}=\alpha(1-c) /(4-\alpha) \equiv x_{R}^{E T H}
$$


for the case of $x_{R} \in\left[K_{H}, \infty\right)$. Comparing the candidate investment levels $x_{R}^{E T L}, x_{R}^{E T M}$, and $x_{R}^{E T H}$ with the threshold values $\bar{x}_{R}^{L}, \bar{x}_{R}^{H}$, and $K_{H}$, we have the following:

$$
\left\{\begin{array}{llll}
x_{R}^{E T L} \in\left[0, \bar{x}_{R}^{L}\right), & x_{R}^{E T M} \leq \bar{x}_{R}^{H}, & x_{R}^{E T H} \leq K_{H} & \text { if } z<z_{l}^{E T}, \\
x_{R}^{E T L} \geq \bar{x}_{R}^{L}, & x_{R}^{E T M} \leq \bar{x}_{R}^{H}, & x_{R}^{E T H}<K_{H} & \text { if } z_{l}^{E T} \leq z \leq z_{m}^{E T}, \\
x_{R}^{E T L}>\bar{x}_{R}^{L}, & x_{R}^{E T M} \in\left(\bar{x}_{R}^{H}, K_{H}\right), & x_{R}^{E T H}<K_{H} & \text { if } z_{m}^{E T}<z<z_{H}^{E T}, \\
x_{R}^{E T L}>\bar{x}_{R}^{L}, & x_{R}^{E T M} \geq K_{H}, & x_{R}^{E T H} \in\left[K_{H}, \infty\right) & \text { if } z \geq z_{H}^{E T},
\end{array}\right.
$$

where

$$
z_{l}^{E T} \equiv \frac{1-\alpha^{2}+J}{5-\alpha-\alpha^{2}}, \quad z_{m}^{E T} \equiv \frac{8-18 \alpha+7 \alpha^{2}-\alpha^{3}-4 \sqrt{(1-\alpha) \alpha}}{16-28 \alpha+9 \alpha^{2}-\alpha^{3}}, \quad z_{H}^{E T} \equiv \frac{2-\alpha}{4-\alpha} .
$$

Moreover, we can show that $\pi_{R}^{E T}\left(K_{H}\right)=\lim _{x_{R} \rightarrow K_{H}-0} \pi_{R}^{E T}\left(x_{R}\right)$ and $\pi_{R}^{E T}\left(x_{R}\right)$ is concave in each interval. Hence, the candidate investment levels for the encroachment interval are $x_{R}^{E T L}$ if $z<z_{l}^{E T}, \bar{x}_{R}^{L}$ and $\bar{x}_{R}^{H}$ if $z_{l}^{E T} \leq z \leq z_{m}^{E T}, x_{R}^{E T M}$ if $z_{m}^{E T}<z<z_{H}^{E T}$, and $x_{R}^{E T H}$ if $z \geq z_{H}^{E T}$.

Next, we consider the investment level without encroachment in the following interval for $x_{R}:\left[\bar{x}_{R}^{L}, \bar{x}_{R}^{H}\right]$. Solving $\partial \pi_{M}^{N T}\left(x_{R}\right) / \partial x_{R}=0$, we have $x_{R}=\alpha(1-c) /(4-\alpha)=x_{R}^{N T}$. Comparing $x_{R}^{N T}$ with the threshold values $\bar{x}_{R}^{L}$ and $\bar{x}_{R}^{H}$, we have the following.

$$
\begin{cases}x_{R}^{N T}<\bar{x}_{R}^{L} & \text { if } z<z^{L}=\frac{4-5 \alpha+4 \alpha^{2}-4 J}{(4-\alpha)(5-4 \alpha)} \\ x_{R}^{N T} \in\left[\bar{x}_{R}^{L}, \bar{x}_{R}^{H}\right] & \text { if } z^{L} \leq z \leq z^{H}, \\ x_{R}^{N T}>\bar{x}_{R}^{H} & \text { if } z>z^{H}=\frac{8-16 \alpha+5 \alpha^{2}-4 \sqrt{(1-\alpha) \alpha}}{(4-\alpha)(4-5 \alpha)} .\end{cases}
$$

Hence, for $x_{R} \in\left[\bar{x}_{R}^{L}, \bar{x}_{R}^{H}\right]$, the candidate investment level is $\bar{x}_{R}^{L}$ if $z<z^{L}, x_{R}^{N T}$ if $z^{L} \leq z \leq z^{H}$, and $\bar{x}_{R}^{H}$ if $z>z^{H}$.

From the two previous cases, we compare the threshold values $z_{l}^{E T}, z_{m}^{E T}, z_{H}^{E T}, z^{L}$, and $z^{H}$ in order to identify the candidate optimal investment levels for each $z$. The comparison leads to the relation $z^{L}<z_{l}^{E T}<z_{m}^{E T}<z^{H}<z_{H}^{E T}$. Hence, the candidate investment levels are as follows: (1) $x_{R}^{E T L}$ and $\bar{x}_{R}^{L}$ if $z<z^{L}$; (2) $x_{R}^{E T L}$ and $x_{R}^{N T}$ if $z^{L} \leq z<z_{l}^{E T}$; (3) $\bar{x}_{R}^{L}$, $\bar{x}_{R}^{H}$, and $x_{R}^{N T}$ if $z_{l}^{E T} \leq z \leq z_{m}^{E T}$; (4) $x_{R}^{E T M}$ and $x_{R}^{N T}$ if $z_{m}^{E T}<z \leq z^{H}$; (5) $x_{R}^{E T M}$ and $\bar{x}_{R}^{H}$ if $z^{H}<z<z_{H}^{E T}$; and (6) $x_{R}^{E T H}$ and $\bar{x}_{R}^{H}$ if $z_{H}^{E T} \leq z$.

We explicitly consider case $(1)\left(z<z^{L}\right)$. After some calculation, we have $\pi_{R}^{E T}\left(x_{R}^{E T L}\right)<$ $\pi_{R}^{N T}\left(\bar{x}_{R}^{L}\right)$. Note that $x_{R}^{E T L}<K_{L}$ and $\bar{x}_{R}^{L}>0$ for any $z<z^{L}$. Hence, the equilibrium 
investment level is $x_{R}=\bar{x}_{R}^{L}$. Applying similar procedures to the other four cases, except case (5) $\left(z^{H}<z<z_{H}^{E T}\right)$, we have that $\pi_{R}^{E T}\left(x_{R}^{E T L}\right)<\pi_{R}^{N T}\left(x_{R}^{N T}\right)$ for $z^{L} \leq z<z_{l}^{E T}, \pi_{R}^{E T}\left(\bar{x}_{R}^{L}\right)<$ $\pi_{R}^{N T}\left(x_{R}^{N T}\right)$ and $\pi_{R}^{E T}\left(\bar{x}_{R}^{H}\right)<\pi_{R}^{N T}\left(x_{R}^{N T}\right)$ for $z_{l}^{E T} \leq z \leq z_{m}^{E T}, \pi_{R}^{E T}\left(x_{R}^{E T M}\right)<\pi_{R}^{N T}\left(x_{R}^{N T}\right)$ for $z_{m}^{E T}<z \leq z^{H}$, and $\pi_{R}^{E T}\left(x_{R}^{E T H}\right)>\pi_{R}^{N T}\left(\bar{x}_{R}^{H}\right)$ for $z_{H}^{E T} \leq z$.

Finally, we consider case (5) $\left(z^{H}<z<z_{H}^{E T}\right)$. Solving $\pi_{R}^{E T}\left(x_{R}^{E T M}\right)<\pi_{R}^{N T}\left(\bar{x}_{R}^{H}\right)$, we have $z<\left(8-15 \alpha+7 \alpha^{2}-\alpha^{3}-2 \sqrt{(1-\alpha) \alpha}\right) /\left(16-25 \alpha+9 \alpha^{2}-\alpha^{3}\right) \equiv z_{h}$. Here, if $R$ can take $x_{R}=\bar{x}_{R}^{H}$, we must keep $\bar{x}_{R}^{H} \geq 0$. Solving this inequality, we have $z \leq$ $(2-3 \alpha-\sqrt{\alpha(1-\alpha)}) /(4-5 \alpha) \equiv z^{x R H}$. Comparing the threshold values $z^{H}, z_{H}^{E T}, z_{h}$, and $z^{x R H}$, we have $z^{H}<z^{x R H}<z_{h}<z_{H}^{E T}$. Then, for $z^{x R H}<z<z_{h}, R$ cannot choose $x_{R}=\bar{x}_{R}^{H}$, which is the candidate of the optimal (corner) solution on the range $\left[\bar{x}_{R}^{L}, \bar{x}_{R}^{H}\right]$, because this range is empty if $z^{x R H}<z$. Hence, the equilibrium investment level in this case is $x_{R}=\bar{x}_{R}^{H}$ if $z^{H}<z \leq z^{x R H}$, and $x_{R}=x_{R}^{E T M}$ if $z^{x R H}<z<z_{H}^{E T}$.

Summarizing the above discussion, for $0 \leq \alpha \leq 1 / 5$, the equilibrium investment level is given as follows:

$$
x_{R}= \begin{cases}\bar{x}_{R}^{L} & \text { if } 0 \leq z<z^{L} \\ x_{R}^{N T} & \text { if } z^{L} \leq z \leq z^{H} \\ \bar{x}_{R}^{H} & \text { if } z^{H}<z \leq z^{x R H} \\ x_{R}^{E T M} & \text { if } z^{z R H}<z<z_{H}^{E T} \\ x_{R}^{E T H} & \text { if } z_{H}^{E T} \leq z\end{cases}
$$

Case (II) In this case, we assume $1 / 5<\alpha \leq 1 / 4$. As in case (I), we can show the following:

$$
\left\{\begin{array}{llll}
x_{R}^{E T L} \in\left[0, \bar{x}_{R}^{L}\right), & x_{R}^{E T M}<K_{L}, & x_{R}^{E T H}<K_{H} & \text { if } z<z_{l}^{E T}, \\
x_{R}^{E T L} \in\left[\bar{x}_{R}^{L}, \bar{x}_{R}^{M}\right], & x_{R}^{E T M}<K_{L}, & x_{R}^{E T H}<K_{H} & \text { if } z_{l}^{E T} \leq z \leq z_{l^{\prime}}^{E T}, \\
x_{R}^{E T L} \in\left(\bar{x}_{R}^{M}, K_{L}\right), & x_{R}^{E T M}<K_{L}, & x_{R}^{E T H}<K_{H} & \text { if } z_{l^{\prime}}^{E T}<z<z_{L}^{E T}, \\
x_{R}^{E T L} \geq K_{L}, & x_{R}^{E T M} \in\left[K_{L}, K_{H}\right), & x_{R}^{E T H}<K_{H} & \text { if } z_{L}^{E T} \leq z<z_{H}^{E T}, \\
x_{R}^{E T L} \geq K_{L}, & x_{R}^{E T M} \geq K_{H}, & x_{R}^{E T H} \in\left[K_{H}, \infty\right) & \text { if } z_{H}^{E T} \leq z,
\end{array}\right.
$$

where $z_{l^{\prime}}^{E T} \equiv\left(1-\alpha^{2}+J\right) /\left(5-\alpha-\alpha^{2}\right)$ and $z_{L}^{E T} \equiv(1-\alpha) /(3-\alpha)$. We can also show that $\pi_{R}^{E T}\left(x_{R}\right)$ is continuous at $x_{R}=K_{L}$ and $K_{H}$. Hence, the candidate investment levels with encroachment are $x_{R}^{E T L}$ if $z<z_{l}^{E T}, \bar{x}_{R}^{L}$ and $\bar{x}_{R}^{M}$ if $z_{l}^{E T} \leq z \leq z_{l^{\prime}}^{E T}, x_{R}^{E T L}$ if $z_{l^{\prime}}^{E T}<z<z_{L}^{E T}$, $x_{R}^{E T M}$ if $z_{L}^{E T} \leq z<z_{H}^{E T}$, and $x_{R}^{E T H}$ if $z \geq z_{H}^{E T}$. 
In the case of no-encroachment, $x_{R}^{N T}$ has the following relation:

$$
\begin{cases}x_{R}^{N T}<\bar{x}_{R}^{L} & \text { if } z<z^{L} \\ x_{R}^{N T} \in\left[\bar{x}_{R}^{L}, \bar{x}_{R}^{M}\right] & \text { if } z^{L} \leq z \leq z^{M} \equiv \frac{4-5 \alpha+4 \alpha^{2}+4 J}{(4-\alpha)(5-4 \alpha)} \\ x_{R}^{N T}>\bar{x}_{R}^{M} & \text { if } z>z^{M}\end{cases}
$$

Hence, the candidate investment level without encroachment is $\bar{x}_{R}^{L}$ if $z<z^{L}, x_{R}^{N T}$ if $z^{L} \leq$ $z \leq z^{M}$, and $\bar{x}_{R}^{M}$ if $z>z^{M}$.

Before we check the relation between the threshold values of $z$, we check the condition that $\bar{x}_{R}^{M} \geq 0$. Then, we have $z \leq(1+J) /(5-4 \alpha) \equiv z^{x R M}$. Thus, if $z>z^{x R M}$, the case of no encroachment never occurs. Here, we have seven threshold values: $z_{l}^{E T}, z_{l^{\prime}}^{E T}$, $z_{L}^{E T}, z_{H}^{E T}, z^{L}, z^{M}$, and $z^{x R M}$. First, comparing these, excluding $z_{L}^{E T}$, we have $z^{L} \leq z_{l}^{E T} \leq$ $z_{l^{\prime}}^{E T} \leq z^{M}<z^{x R M}<z_{H}^{E T}$. Next, we compare $z_{L}^{E T}$ with these six threshold values. Then, we have $z^{L}<z_{l}^{E T}<z_{l^{\prime}}^{E T} \leq z_{L}^{E T} \leq z^{M}<z^{x R M}<z_{H}^{E T}$ if $1 / 5<\alpha \leq 2(5-3 \sqrt{2}) / 7(\approx 0.216)$, $z^{L} \leq z_{l}^{E T} \leq z_{l^{\prime}}^{E T} \leq z^{M}<z_{L}^{E T}<z^{x R M}<z_{H}^{E T}$ if $2(5-3 \sqrt{2}) / 7<\alpha \leq 0.247$, and $z^{L} \leq z_{l}^{E T} \leq$ $z_{l^{\prime}}^{E T} \leq z^{M}<z^{x R M}<z_{L}^{E T}<z_{H}^{E T}$ if $0.247<\alpha \leq 1 / 4 .^{11}$

\footnotetext{
11 The value of 0.247 is obtained by numerically by solving $z^{x R M}=z_{L}^{E T}$ for $\alpha$.
} 
- Case with $\alpha \in(1 / 5,0.216]$

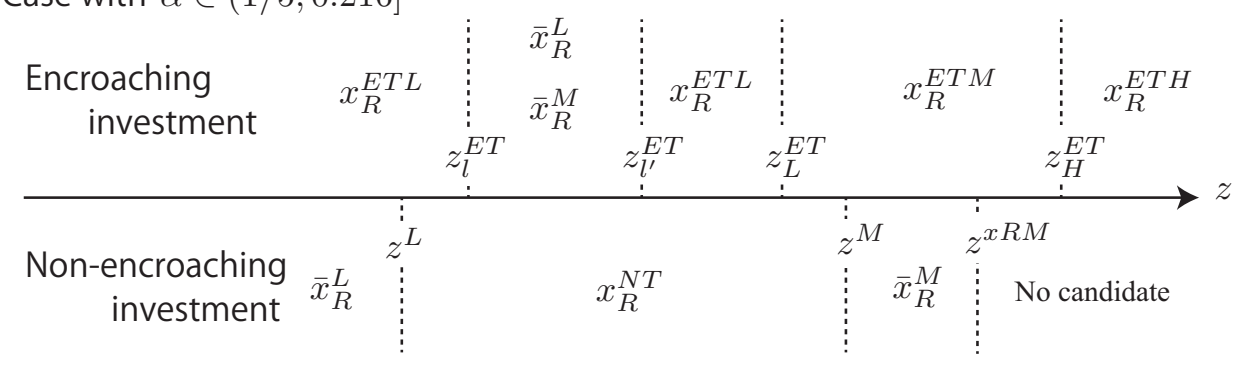

- Case with $\alpha \in(0.216,0.247]$
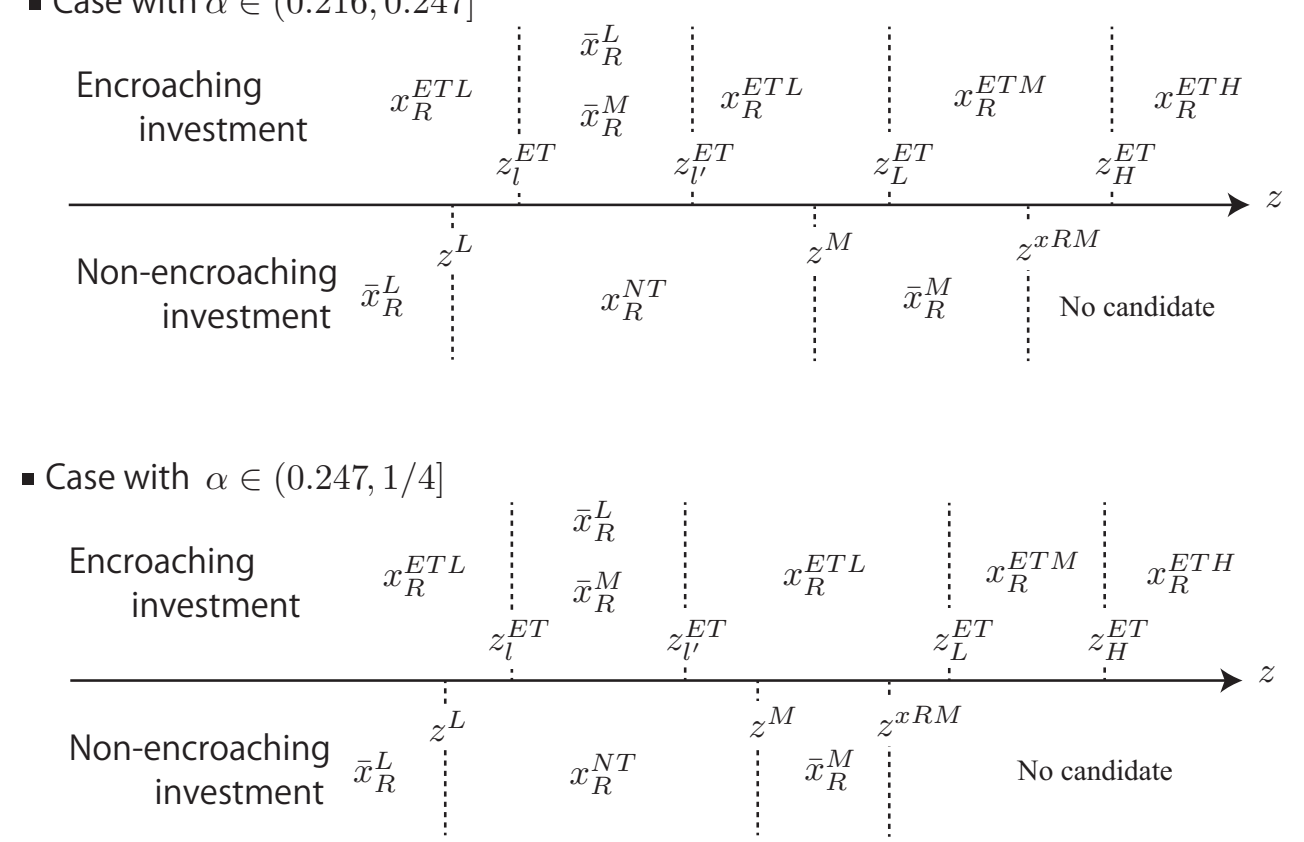

Figure A1: Candidates of optimal investment level

From the above discussion, we can identify the candidate optimal investment levels, as shown in Figure A1. We have three cases, based on $\alpha$. The horizontal axis is the value of $z$. On the upper part of each axis in Figure A1, we describe the candidate optimal investment levels leading to encroachment. On the lower part of each axis in Figure A1, we describe the candidates of optimal investment level leading to non-encroachment.

We compare the candidates in each region for $z$. First, when $z \leq \min \left\{z_{L}^{E T}, z^{M}\right\}$, we have the same comparisons for the candidates. Then, for any $\alpha \in(1 / 5,1 / 4]$, we have $\pi_{R}^{E T}\left(x_{R}^{E T L}\right)<\pi_{R}^{N T}\left(\bar{x}_{R}^{L}\right)$ for $z \in\left[0, z^{L}\right), \pi_{R}^{E T}\left(x_{R}^{E T L}\right)<\pi_{R}^{N T}\left(x_{R}^{N T}\right)$ for $z \in\left[z^{L}, z_{l}^{E T}\right), \pi_{R}^{E T}\left(\bar{x}_{R}^{L}\right)<$ $\pi_{R}^{N T}\left(x_{R}^{N T}\right)$ and $\pi_{R}^{E T}\left(\bar{x}_{R}^{M}\right)<\pi_{R}^{N T}\left(x_{R}^{N T}\right)$ for $z \in\left[z_{l}^{E T}, z_{l^{\prime}}^{E T}\right]$, and $\pi_{R}^{E T}\left(x_{R}^{E T L}\right)<\pi_{R}^{N T}\left(x_{R}^{N T}\right)$ for 
$z \in\left(z_{l^{\prime}}^{E T}, \min \left\{z_{L}^{E T}, z^{M}\right\}\right)$. Moreover, if $z>z^{x R M}$, there is one candidate. Hence, the optimal investment is as follows. For $\alpha \in(1 / 5,1 / 4]$, the optimal investment is $x_{R}^{E T H}$ if $z>z_{H}^{E T}$. For $\alpha \in(1 / 5,0.247]$, the optimal investment is $x_{R}^{E T M}$ if $z \in\left(z^{x R M}, z_{H}^{E T}\right]$. For $\alpha \in(0.247,1 / 4]$, the optimal investment is $x_{R}^{E T L}$ if $z \in\left(z^{x R M}, z_{L}^{E T}\right]$, and $x_{R}^{E T M}$ if $z \in\left(z_{L}^{E T}, z_{H}^{E T}\right]$.

The remaining comparisons are for the following three cases: $\alpha \in(1 / 5,0.216]$ and $z \in\left[z_{L}^{E T}, z^{M}\right) ; \alpha \in(1 / 5,0.247]$ and $z \in\left[\max \left\{z_{L}^{E T}, z^{M}\right\}, z^{x R M}\right] ;$ and $\alpha \in(0.216,1 / 4]$ and $z \in\left[z^{M}, \min \left\{z_{L}^{E T}, z^{x R M}\right\}\right)$. For the first case, we have $\pi_{R}^{E T}\left(x_{R}^{E T M}\right)<\pi_{R}^{N T}\left(x_{R}^{N T}\right)$; for the second case, we have $\pi_{R}^{E T}\left(x_{R}^{E T M}\right)<\pi_{R}^{N T}\left(\bar{x}_{R}^{M}\right)$; and for the third case, we have $\pi_{R}^{E T}\left(x_{R}^{E T L}\right)<\pi_{R}^{N T}\left(\bar{x}_{R}^{M}\right)$.

Summarizing the above discussion, we obtain the equilibrium investment level for $1 / 5<$ $\alpha \leq 0.247$ as follows:

$$
x_{R}= \begin{cases}\bar{x}_{R}^{L} & \text { if } 0 \leq z<z^{L}, \\ x_{R}^{N T} & \text { if } z^{L} \leq z \leq z^{M}, \\ \bar{x}_{R}^{M} & \text { if } z^{M}<z \leq z^{x R M} \\ x_{R}^{E T M} & \text { if } z^{x R M}<z<z_{H}^{E T}, \\ x_{R}^{E T H} & \text { if } z_{H}^{E T} \leq z\end{cases}
$$

For the case of $0.247<\alpha \leq 1 / 4$, we have

$$
x_{R}= \begin{cases}\bar{x}_{R}^{L} & \text { if } 0 \leq z<z^{L}, \\ x_{R}^{N T} & \text { if } z^{L} \leq z \leq z^{M}, \\ \bar{x}_{R}^{M} & \text { if } z^{M}<z \leq z^{x R M}, \\ x_{R}^{E T L} & \text { if } z^{x R M}<z \leq z_{L}^{E T}, \\ x_{R}^{E T M} & \text { if } z_{L}^{E T}<z<z_{H}^{E T}, \\ x_{R}^{E T H} & \text { if } z_{H}^{E T} \leq z .\end{cases}
$$

Case (III) Finally, we consider the case of $\alpha>1 / 4$. As in cases (I) and (II), we can show the following:

$$
\left\{\begin{array}{llll}
x_{R}^{E T L} \in\left[0, K_{L}\right), & x_{R}^{E T M}<K_{L}, & x_{R}^{E T H}<K_{H} & \text { if } z<z_{L}^{E T}, \\
x_{R}^{E T L}>K_{L}, & x_{R}^{E T M} \in\left[K_{L}, K_{H}\right), & x_{R}^{E T H}<K_{H} & \text { if } z_{L}^{E T} \leq z<z_{H}^{E T}, \\
x_{R}^{E T L}>K_{L}, & x_{R}^{E T M}>K_{H}, & x_{R}^{E T H} \in\left[K_{H}, \infty\right) & \text { if } z \geq z_{H}^{E T} .
\end{array}\right.
$$

Because $\pi_{R}^{E T}\left(x_{R}\right)$ is continuous and concave, the optimal investment level is

$$
x_{R}= \begin{cases}x_{R}^{E T L} & \text { if } z \leq z_{L}^{E T} \\ x_{R}^{E T M} & \text { if } z_{L}^{E T}<z<z_{H}^{E T}, \\ x_{R}^{E T H} & \text { if } z_{H}^{E T} \leq z\end{cases}
$$


Integrating cases (I), (II), and (III), we obtain the equilibrium investment level $x_{R}^{T *}$ in (22) and (23) in Proposition 4. We summarize the condition that $M$ opens its own direct channel in (23) in Proposition 4.

For the encroachment regions where the equilibrium investment level is $x_{R}^{E T L}, x_{R}^{E T M}$, or $x_{R}^{E T H}$, substituting these into $q_{M}^{E}\left(x_{R}, w^{E T}\left(x_{R}\right)\right), q_{R}^{E}\left(x_{R}, w^{E T}\left(x_{R}\right)\right), w^{E T}\left(x_{R}\right), f^{E T}\left(x_{R}\right)$, $\pi_{M}^{E T}\left(x_{R}\right)$, and $\pi_{R}^{E T}\left(x_{R}\right)$, we obtain the equilibrium outcomes. Note that we have $x_{R}^{E T L}<$ $K_{L} \leq x_{R}^{E T M}<K_{H} \leq x_{R}^{E T H}$. Similarly, for the non-encroachment region where the equilibrium investment level is $\bar{x}_{R}^{L}, \bar{x}_{R}^{M}, \bar{x}_{R}^{H}$, or $x_{R}^{N T}$, substituting these into $q_{R}^{N}\left(x_{R}, 0\right), f^{N T}\left(x_{R}\right)$, $\pi_{M}^{N T}\left(x_{R}\right)$, and $\pi_{R}^{N T}\left(x_{R}\right)$, we obtain the equilibrium outcomes where $q_{M}^{N}=0$ and $w^{N T}=0$. From the above outcomes, we obtain $C S^{T *}=\left(q_{M}^{T *}+q_{R}^{T *}\right)^{2} / 2$ and $T S^{T *}=C S^{T *}+\pi_{R}^{T *}+\pi_{M}^{T *}$, where the superscript $T *$ denotes the equilibrium outcomes. Moreover, we draw these outcomes with $z$ as a variable. For $\alpha=0.1,0.22$, and 0.28 , we obtain the four curves of the equilibrium outcome shown in Figure 5.

\section{B.2 Cost-reduction after encroachment}

We consider the case of a cost-reduction after an encroachment decision. Using the results in the previous discussion, we easily obtain the subgame outcomes after Stage 1. In the encroachment case, the investment level is equal to that in case (III). Substituting $x_{R}^{E T}$ in (23) into $\pi_{M}^{E T}\left(x_{R}\right)$, we obtain the profit of $M$, as in subsection 4.5.

$$
\pi_{M}^{E T}\left(x_{R}^{E T}\right)= \begin{cases}\frac{(1-c)^{2}\left((1-\alpha)(1-z)^{2}+4 z^{2}\right)}{4(1-\alpha)}, & \text { if } z<z_{L}^{E T}, \\ \frac{(1-c)^{2}(1-z)\left(\alpha(3-2 \alpha)+\left(4-7 \alpha+2 \alpha^{2}\right) z\right)}{(1-c)^{2}\left(\alpha(4-\alpha)^{2}(1-z)^{2}+16(1-\alpha)\right)}, & \text { if } z_{L}^{E T} \leq z<z_{H}^{E T}, \\ \frac{(4-\alpha)^{2}}{2 T}, & \text { if } z_{L}^{E T} \leq z .\end{cases}
$$

For the non-encroachment case, substituting $x_{R}=x_{R}^{N T}$ into $\pi_{M}^{N T}\left(x_{R}\right)$, we have

$$
\pi_{M}^{N T}\left(x_{R}^{N T}\right)=\frac{4(1-\alpha)(1-c)^{2}}{(4-\alpha)^{2}} .
$$

Because there are two threshold values $z_{L}^{E T}$ and $z_{H}^{E T}$, we consider three cases: $z<z_{L}^{E T}$, $z_{L}^{E T} \leq z<z_{H}^{E T}$, and $z_{L}^{E T} \leq z$. We consider the first case, $z<z_{L}^{E T}$. Solving $\pi_{M}^{E T}\left(x_{R}^{E T}\right)-$ 
$\pi_{M}^{N T}\left(x_{R}^{N T}\right)>0$, we obtain the encroachment condition, as follows:

$$
\begin{cases}z<\frac{(4-\alpha)(1-\alpha)-2 \sqrt{(1-\alpha)\left(4-16 \alpha+3 \alpha^{2}\right)}}{(5-\alpha)(4-\alpha)} \equiv \bar{z}_{M}^{L} & \text { if } 0 \leq \alpha<0.159, \\
\left(\begin{array}{cc}
z<\bar{z}_{M}^{L} \text { or } & \text { if } 0.159 \leq \alpha<0.263, \\
z>\frac{(4-\alpha)(1-\alpha)+2 \sqrt{(1-\alpha)\left(4-16 \alpha+3 \alpha^{2}\right)}}{(5-\alpha)(4-\alpha)} \equiv \bar{z}_{M}^{H 1}
\end{array}\right) & \text { if } \alpha \geq 0.263 \approx \frac{2(4-\sqrt{13})}{3} .\end{cases}
$$

Second, for $z_{L} \leq z<z_{H}^{E T}$, and solving $\pi_{M}^{E T}\left(x_{R}^{E T}\right)-\pi_{M}^{N T}\left(x_{R}^{N T}\right)>0$, we have the following encroachment condition:

$$
\begin{cases}z>\frac{(4-\alpha)(2-\alpha)(1-2 \alpha)-2 \sqrt{\alpha(1-\alpha)\left(4+\alpha-\alpha^{2}\right)}}{(4-\alpha)\left(4-7 \alpha+2 \alpha^{2}\right)} \equiv \bar{z}_{M}^{H 2} & \text { if } 0 \leq \alpha<0.159, \\ \text { for any } z & \text { if } 0.159 \leq \alpha \leq 1 .\end{cases}
$$

Finally, for $z_{H}^{E T} \leq z$, we consider $\pi_{M}^{E T}\left(x_{R}^{E T}\right)-\pi_{M}^{N T}\left(x_{R}^{N T}\right)$, which we find always takes a positive value. Hence, for any $z$, the encroachment condition is satisfied.

Therefore, integrating the results in the three cases (define $\bar{z}_{M}^{H} \equiv \max \left\{\bar{z}_{M}^{H 1}, \bar{z}_{M}^{H 2}\right\}$ as in (28)), we obtain Proposition 5. As in the previous subsection, by substituting the equilibrium outcomes into each stage, we can draw the equilibrium outcomes, as shown in Figure 7. Note that in the encroachment case, the equilibrium investment function takes a different form based on the value of $z$, with threshold values $z_{L}^{E T}$ and $z_{H}^{E T}$.

\section{Proof of Proposition 6}

In this section, we prove Proposition 6. From (16)-(19), we have

$$
\begin{gathered}
\pi_{R}^{N T}\left(x_{R}\right)=\frac{\alpha\left(1-c+x_{R}\right)^{2}}{4}-x_{R}^{2}, \\
\pi_{M}^{N T}\left(x_{R}\right)=\frac{(1-\alpha)\left(1-c+x_{R}\right)^{2}}{4}, \\
\pi_{R}^{E T}\left(x_{R}\right)= \begin{cases}\alpha\left((1-c) z+x_{R}\right)^{2}-x_{R}^{2} . & \text { if } x_{R}<K_{L}, \\
\frac{(1-c)(1-z)\left(-(1-c)(1-5 z)+4 x_{R}\right)}{4}-x_{R}^{2} & \text { if } K_{L} \leq x_{R}<K_{H}, \\
\frac{\alpha\left((1-c) z+x_{R}\right)\left((1-c)(2-z)+x_{R}\right)}{4}-x_{R}^{2} & \text { if } K_{H} \leq x_{R},\end{cases}
\end{gathered}
$$




$$
\pi_{M}^{E T}\left(x_{R}\right)= \begin{cases}\frac{(1-c)^{2}(1-z)^{2}+4(1-\alpha)\left((1-c) z+x_{R}\right)^{2}}{4} & \text { if } x_{R}<K_{L}, \\ \frac{(1-c)(1-z)\left((1-c)(\alpha+(4-5 \alpha) z)+4(1-\alpha) x_{R}\right)}{4} & \text { if } K_{L} \leq x_{R}<K_{H}, \\ \frac{(1-\alpha)\left(1-c+x_{R}\right)^{2}+\alpha(1-c)^{2}(1-z)^{2}}{4} & \text { if } K_{H} \leq x_{R} .\end{cases}
$$

The consumer surplus in the non-encroachment case and that in the encroachment case in stage 3 are respectively given as

$$
\begin{aligned}
& C S^{N T}\left(x_{R}\right)= \frac{\left(1-c+x_{R}\right)^{2}}{8}, \\
& C S^{E T}\left(x_{R}\right)= \begin{cases}\frac{\left(1-c+c_{M}+2 x_{R}\right)^{2}}{8} & \text { if } x_{R}<K_{L}, \\
\frac{\left(1-c-c_{M}\right)^{2}}{2} & \text { if } K_{L} \leq x_{R}<K_{H}, \\
\frac{\left.2-2 c-c_{M}+x_{R}\right)^{2}}{18} & \text { if } K_{H} \leq x_{R} .\end{cases}
\end{aligned}
$$

Then, the total surplus in the non-encroachment case and that in the encroachment case in stage 3 are respectively given as $T S^{N T}\left(x_{R}\right)=\pi_{R}^{N T}\left(x_{R}\right)+\pi_{M}^{N T}\left(x_{R}\right)+C S^{N T}\left(x_{R}\right)$ and $T S^{E T}\left(x_{R}\right)=\pi_{R}^{E T}\left(x_{R}\right)+\pi_{M}^{E T}\left(x_{R}\right)+C S^{E T}\left(x_{R}\right)$.

From Propositions 4 and 5, we have the following nine threshold values for $z: \bar{z}_{M}^{L}, \bar{z}_{M}^{H}$, $z^{L}, z^{M}, z^{H}, z^{x R M}, z^{x R H}, z_{L}^{E T}$, and $z_{H}^{E T}$. Integrating Figures 4 and 6 , we have Figure A2.

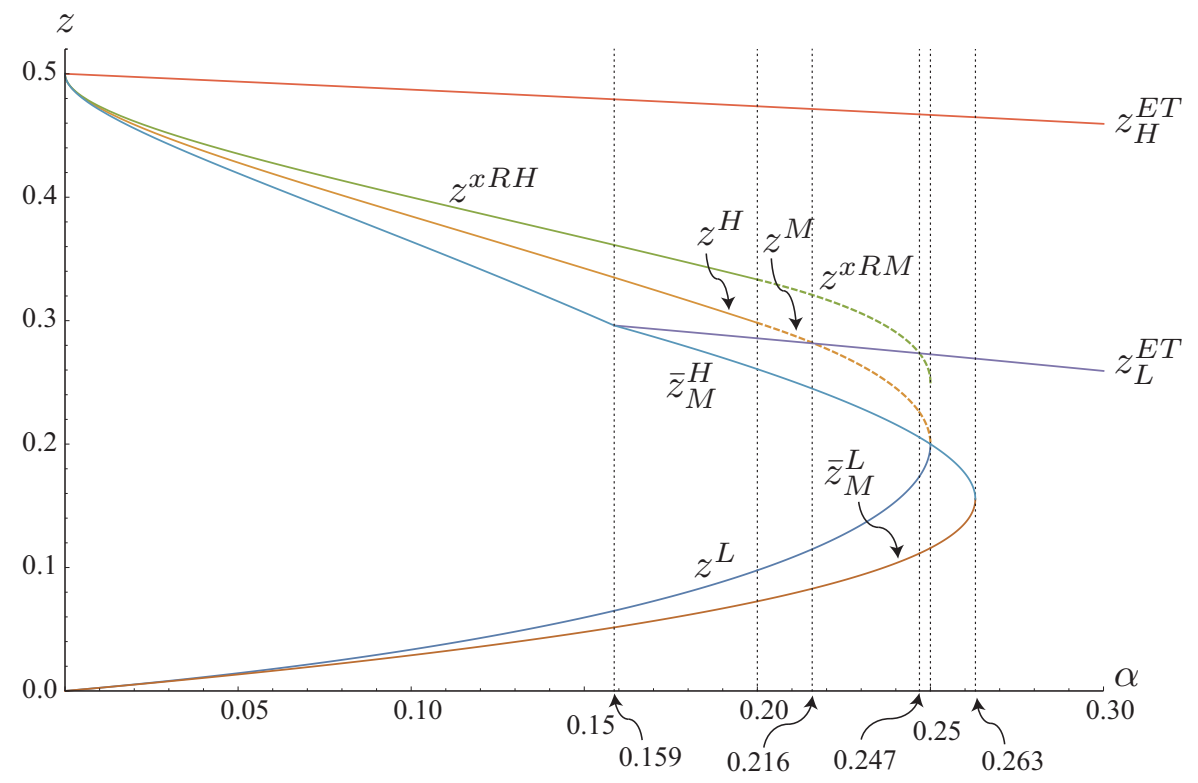

Figure A2: The threshold values for $z$ in Sections 4.4 and 4.5 
From Figure A2, we have the following inequalities. ${ }^{12}$

$$
\begin{array}{rcc}
\bar{z}_{M}^{L}<z^{L}<\bar{z}_{M}^{H}<z^{H}<z^{x R H}<z_{H}^{E T} & \text { if } & \alpha \in[0,0.159), \\
\bar{z}_{M}^{L}<z^{L}<\bar{z}_{M}^{H}<z_{L}^{E T}<z^{H}<z^{x R H}<z_{H}^{E T} & \text { if } & \alpha \in[0.159,1 / 5), \\
\bar{z}_{M}^{L}<z^{L}<\bar{z}_{M}^{H}<z_{L}^{E T}<z^{M}<z^{x R M}<z_{H}^{E T} & \text { if } & \alpha \in[1 / 5,0.216), \\
\bar{z}_{M}^{L}<z^{L}<\bar{z}_{M}^{H}<z^{M}<z_{L}^{E T}<z^{x R M}<z_{H}^{E T} & \text { if } & \alpha \in[0.216,0.247), \\
\bar{z}_{M}^{L}<z^{L}<\bar{z}_{M}^{H}<z^{M}<z^{x R M}<z_{L}^{E T}<z_{H}^{E T} & \text { if } & \alpha \in[0.247,1 / 4), \\
\bar{z}_{M}^{L}<\bar{z}_{M}^{H}<z_{L}^{E T}<z_{H}^{E T} & \text { if } & \alpha \in[1 / 4,0.263) .
\end{array}
$$

Note that we do not need to consider the case of $\alpha>0.263$ because sections 4.4 and 4.5 have the same equilibrium outcomes for the case. From $x_{R}^{E T}$ in equations (23) and (25) in Sections 4.4 and 4.5, when $M$ encroaches, the investment level is equal to either $x_{R}^{E T L}=\alpha(1-c) z /(1-\alpha), x_{R}^{E T M}=\alpha(1-c)(1-z) / 2$, or $x_{R}^{E T H}=\alpha(1-c) /(4-\alpha)$, regardless of the timing structures of the games. Moreover, we find that $x_{R}^{E T L}<K_{L}$, $K_{L} \leq x_{R}^{E T M}<K_{H}$, and $K_{H} \leq x_{R}^{E T H}$ because the three investment levels are the interior solutions for the intervals of $x_{R},\left[0, K_{L}\right),\left[K_{L}, K_{H}\right)$ and $\left[K_{H}, \infty\right)$, respectively. Hence, we can calculate subgame outcomes from the investment level.

From the above discussion, we can identify the outcomes, as shown in Figures A3-1 and A3-2. We have the following six cases, based on $\alpha$. The horizontal axis is the value of $z$. On the upper part of each axis in Figures A3-1 and A3-2, we describe the outcomes in Section $4.4(R \& D \rightarrow E)$. Below the lower part of each axis in Figures A3-1 and A3-2, we describe the outcomes in Section $4.5(E \rightarrow R \& D)$.

From Figure A3-1 and A3-2, under some parameter ranges, the outcomes in Sections 4.4 and 4.5 are completely the same. More concretely, the ranges are $\alpha \in[0,1 / 4)$ and $z \in\left(z^{L}, \bar{z}_{M}^{H}\right] ; \alpha \in[0,1 / 5)$ and $z \geq z^{x R H} ; \alpha \in[1 / 5,1 / 4)$ and $z \geq z^{x R M} ; \alpha \in[1 / 4,0.263)$ and $\left(z \in\left(0, \bar{z}_{M}^{L}\right)\right.$ or $\left.z \geq \bar{z}_{M}^{H}\right)$; and $\alpha \geq 0.263$. Hence, under the parameter ranges, the timing structures in Sections 4.4 and 4.5 do not influence $M$ 's decision of whether to encroach.

\footnotetext{
12 The values of $\alpha, 0.159,0.216,0.247$, and 0.263 , are obtained by numerically solving $\bar{z}_{M}^{H}=z_{L}^{E T}$, $z^{M}=z_{L}^{E T}, z^{H}=z^{L}$, and $\bar{z}_{M}^{H}=\bar{z}_{M}^{L}$, respectively.
} 
$\alpha \in[0,0.159)$

\begin{tabular}{|c|c|c|c|c|c|c|}
\hline & & & & & $z^{x R H}$ & $\begin{array}{l}E T \\
H\end{array}$ \\
\hline & $\pi_{M}^{N T}$ & & $\pi_{M}^{N T}\left(x_{R}^{N T}\right)$ & $\pi_{M}^{N T}\left(\bar{x}_{R}^{H}\right)$ & $\pi_{M}^{E T}\left(x_{R}^{E T M}\right)$ & $\pi_{M}^{E T}\left(x_{R}^{E T H}\right)$ \\
\hline, 0.159$)$ & $\pi_{R}^{N T}$ & & $\pi_{R}^{N T}\left(x_{R}^{N T}\right)$ & $\pi_{R}^{N T}\left(\bar{x}_{R}^{H}\right)$ & $\pi_{R}^{E T}\left(x_{R}^{E T M}\right)$ & $\pi_{R}^{E T}\left(x_{R}^{E T H}\right)$ \\
\hline & $C S^{N}$ & & $C S^{N T}\left(x_{R}^{N T}\right)$ & $C S^{N T}\left(\bar{x}_{R}^{H}\right)$ & $C S^{E T}\left(x_{R}^{E T M}\right)$ & $C S^{E T}\left(x_{R}^{E T H}\right)$ \\
\hline$R \& D \rightarrow E$ & $T S^{N}$ & & $T S^{N T}\left(x_{R}^{N T}\right)$ & $T S^{N T}\left(\bar{x}_{R}^{H}\right)$ & $T S^{E T}\left(x_{R}^{E T M}\right)$ & $T S^{E T}\left(x_{R}^{E T H}\right)$ \\
\hline & $\pi_{M}^{E T}\left(x_{R}^{E T L}\right)$ & & $\left.R^{N T}\right)$ & $\pi_{M}^{E T}$ & & $\pi_{M}^{E T}\left(x_{R}^{E T H}\right)$ \\
\hline$E \rightarrow R \& D$ & $\pi_{R}^{E T}\left(x_{R}^{E T L}\right)$ & & $v^{2}$ ) & $\pi_{R}^{E T}(2$ & & $\pi_{R}^{E T}\left(x_{R}^{E T H}\right)$ \\
\hline & $C S^{E T}\left(x_{R}^{E T L}\right)$ & $C S$ & $\left.x_{R}^{N T}\right)$ & $C S^{E T}$ & $\left.E^{T T M}\right)$ & $C S^{E T}\left(x_{R}^{E T H}\right)$ \\
\hline & $T S^{E T}\left(x_{R}^{E T L}\right)$ & $T S$ & $\left.x_{R}^{N T}\right)$ & $T S^{E T}$ & $\left.{ }_{R} T M\right)$ & $T S^{E T}\left(x_{R}^{E T H}\right)$ \\
\hline
\end{tabular}

$\alpha \in[0.159,1 / 5)$

\begin{tabular}{|c|c|c|c|}
\hline$z^{L}$ & & $z^{x R H}$ & $\begin{array}{l}E T \\
H\end{array}$ \\
\hline${ }_{M}^{N T}\left(\bar{x}_{R}^{L}\right)$ & $\pi_{M}^{N T}\left(x_{R}^{N T}\right)$ & $\pi_{M}^{N T}\left(\bar{x}_{R}^{H}\right) \pi_{M}^{E T}\left(x_{R}^{E T M}\right)$ & $\pi_{M}^{E T}\left(x_{R}^{E T H}\right)$ \\
\hline${ }_{R}^{N T}\left(\bar{x}_{R}^{L}\right)$ & $\pi_{R}^{N T}\left(x_{R}^{N T}\right)$ & $\pi_{R}^{N T}\left(\bar{x}_{R}^{H}\right): \pi_{R}^{E T}\left(x_{R}^{E T M}\right)$ & $\pi_{R}^{E T}\left(x_{R}^{E T H}\right)$ \\
\hline$N T\left(\bar{x}_{R}^{L}\right)$ & $C S^{N T}\left(x_{R}^{N T}\right)$ & $C S^{N T}\left(\bar{x}_{R}^{H}\right) C S^{E T}\left(x_{R}^{E T M}\right)$ & $C S^{E T}\left(x_{R}^{E T H}\right)$ \\
\hline$N T\left(\bar{x}_{R}^{L}\right)$ & $T S^{N T}\left(x_{R}^{N T}\right)$ & $T S^{N T}\left(\bar{x}_{R}^{H}\right) T S^{E T}\left(x_{R}^{E T M}\right)$ & $T S^{E T}\left(x_{R}^{E T H}\right)$ \\
\hline$\pi_{M}^{N T}\left(x_{R}^{N T}\right)$ & $\pi_{M}^{E T}\left(x_{R}^{E T L}\right)$ & $\pi_{M}^{E T}\left(x_{R}^{E T M}\right)$ & $\pi_{M}^{E T}\left(x_{R}^{E T H}\right)$ \\
\hline$\pi_{R}^{N T}\left(x_{R}^{N T}\right)$ & $\pi_{R}^{E T}\left(x_{R}^{E T L}\right)$ & $\pi_{R}^{E T}\left(x_{R}^{E T M}\right)$ & $\pi_{R}^{E T}\left(x_{R}^{E T H}\right)$ \\
\hline$C S^{N T}\left(x_{R}^{N T}\right.$ & $C S^{E T}\left(x_{R}^{E T L}\right)$ & $C S^{E T}\left(x_{R}^{E T M}\right)$ & $C S^{E T}\left(x_{R}^{E T H}\right)$ \\
\hline & 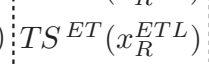 & $T S^{E T}\left(x_{R}^{E T M}\right)$ & $T S^{E T}\left(x_{R}^{E T H}\right)$ \\
\hline $\bar{z}_{M}^{L}$ & H & & \\
\hline
\end{tabular}

$\alpha \in[1 / 5,0.216)$

$$
R \& D \rightarrow E
$$$$
\pi_{M}^{N T}\left(\bar{x}_{R}^{L}\right)
$$

$R \& D \rightarrow E$

$T S^{N T}\left(\bar{x}_{R}^{L}\right)$

$$
E \rightarrow R \& D
$$

$$
\begin{aligned}
& \pi_{M}^{E T}\left(x_{R}^{E T L}\right) \\
& \pi_{R}^{E T}\left(x_{R}^{E T L}\right)
\end{aligned}
$$$$
C S^{E T}\left(x_{R}^{E T L}\right)
$$$$
T S^{E T}\left(x_{R}^{E T}\right)
$$

$$
\bar{z}_{M}^{L}
$$

$$
\bar{z}_{M}^{H}
$$

$$
z_{H}
$$

$$
\pi_{M}^{N T}\left(\bar{x}_{R}^{L}\right)
$$$$
\pi_{R}^{N T}\left(\bar{x}_{R}^{L}\right)
$$$$
C S^{N T}\left(\bar{x}_{R}^{L}\right)
$$

$R \& D \rightarrow E$ $\operatorname{TS}^{N T}\left(\bar{x}_{R}^{L}\right)$<smiles>[C]1CCC1</smiles>

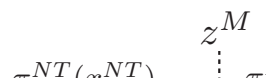

$z^{x R M}$

$z_{H}^{E T}$

$\begin{array}{l:l:l}\pi_{M}^{N T}\left(\bar{x}_{R}^{M}\right) & \pi_{M}^{E T}\left(x_{R}^{E T M}\right) & \pi_{M}^{E T}\left(x_{R}^{E T H}\right) \\ \pi_{R} T\left(x_{R}\right) & \pi_{R}\left(x_{R}\right) & \pi_{R}^{E T}\left(x_{R}^{E T H}\right)\end{array}$ \begin{tabular}{l|l:l:l}
$\pi_{R}^{N T}\left(x_{R}^{N T}\right)$ & $\pi_{R}^{N T}\left(\bar{x}_{R}^{M}\right)$ & $\pi_{R}^{E T}\left(x_{R}^{E T M}\right)$ & $\pi_{R}^{E T}\left(x_{R}^{E T H}\right)$
\end{tabular} $\begin{array}{lll:l}C S^{N T}\left(x_{R}^{N T}\right) & C S^{N T}\left(\bar{x}_{R}^{M}\right) & C S^{E T}\left(x_{R}^{E T M}\right) & C S^{E T}\left(x_{R}^{E T H}\right)\end{array}$

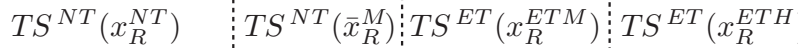

$\begin{array}{c:c}\pi_{M}^{E T}\left(x_{R}^{E T M}\right) & \pi_{M}^{E T}\left(x_{R}^{E T H}\right) \\ \pi_{R}^{E T}\left(x_{R}^{E T M}\right) & \pi_{R}^{E T}\left(x_{R}^{E T H}\right) \\ C S^{E T}\left(x_{R}^{E T M}\right) & C S^{E T}\left(x_{R}^{E T H}\right) \\ T S^{E T}\left(x^{E T M}\right) & T S^{E T}\left(x^{E T H}\right) \\ & z_{H}^{E T}\end{array}$

Figure A3-1: Comparisons of the outcomes in Sections 4.4 and 4.5 


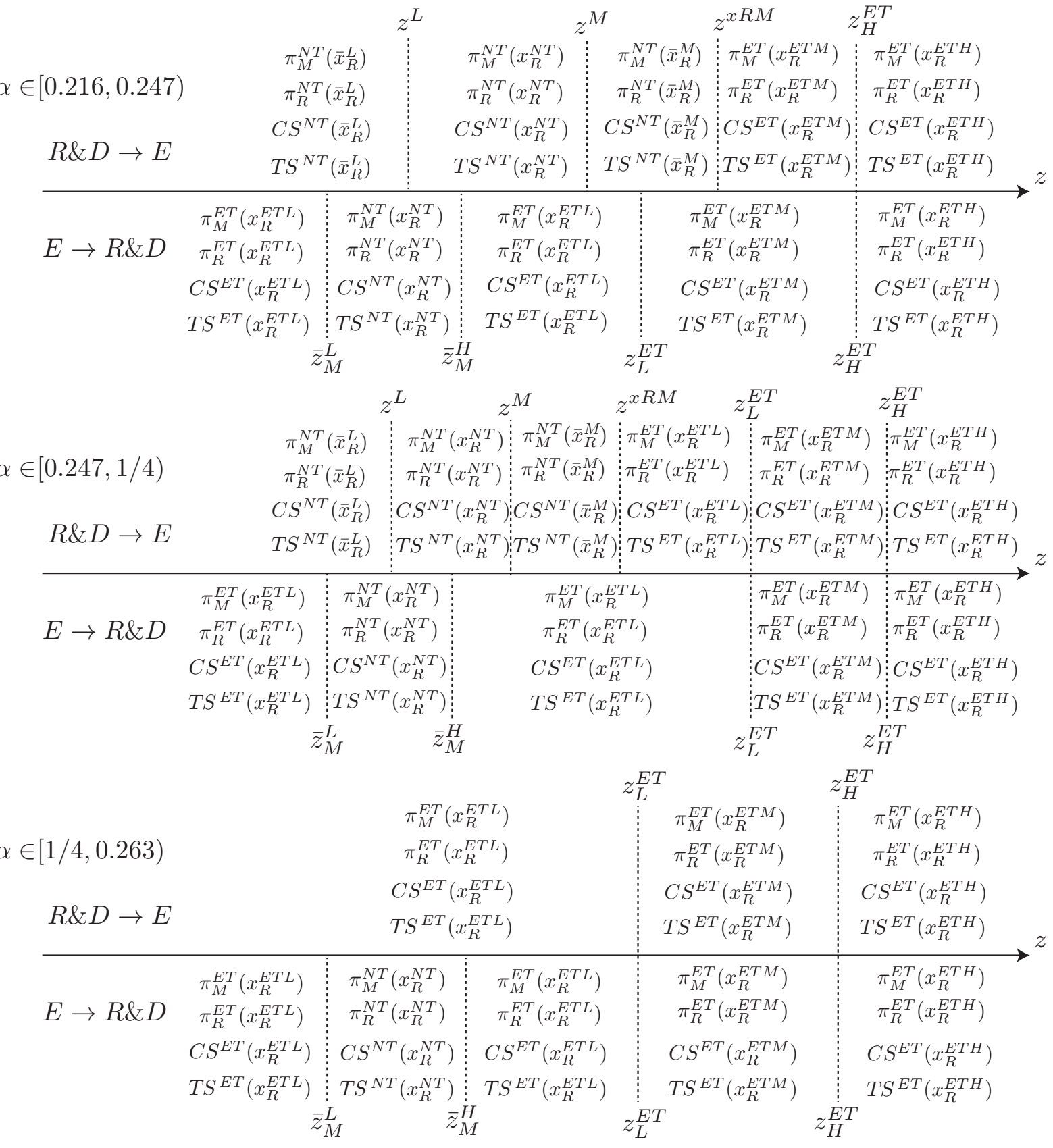

Figure A3-2: Comparisons of the outcomes in Sections 4.4 and 4.5 
In the following discussion, we compare the subgame outcomes in Sections 4.4 and 4.5. First, we show the following two comparisons which are a little bit complex: (i) for $\alpha \in$ $[0,1 / 4)$ and $z \in\left(0, \bar{z}_{M}^{L}\right]$, we compare the consumer surplus in Section 4.4 with that in Section 4.5; (ii) for $\alpha \in[1 / 4,0.263)$ and $z \in\left[\bar{z}_{M}^{L}, \bar{z}_{M}^{H}\right)$, we compare the total surplus in Section 4.4 with that in Section 4.5. Finally, we explain how we compare the subgame outcomes of the other cases in Sections 4.4 and 4.5 .

Case (i) Since at $\alpha=0$, we have $\bar{z}_{M}^{L}=0$. Hence, we do not need to consider the case with $\alpha=0$. Here, we consider the interval $\alpha \in(0,1 / 4)$.

We compare $C S^{N T}\left(\bar{x}_{R}^{L}\right)$ with $C S^{E T}\left(x_{R}^{E T L}\right)$. The difference $C S^{N T}\left(\bar{x}_{R}^{L}\right)-C S^{E T}\left(x_{R}^{E T L}\right)$ is

$$
C S^{N T}\left(\bar{x}_{R}^{L}\right)-C S^{E T}\left(x_{R}^{E T L}\right)=\Phi_{2}^{C S} z^{2}+\Phi_{1}^{C S} z+\Phi_{0}^{C S}
$$

where

$$
\begin{aligned}
\Phi_{2}^{C S} & \equiv-\frac{9(1+\alpha)^{2}+[4(1-\alpha)-\sqrt{(1-\alpha)(1-4 \alpha)}]^{2}}{72(1-\alpha)^{2}}(<0), \\
\Phi_{1}^{C S} & \equiv-\frac{26-11 \alpha-8 \sqrt{(1-\alpha)(1-4 \alpha)}}{36(1-\alpha)}(<0), \\
\Phi_{0}^{C S} & \equiv \frac{8-11 \alpha-8 \sqrt{(1-\alpha)(1-4 \alpha)}}{72(1-\alpha)}(>0) .
\end{aligned}
$$

The difference is a quadratic function of $z$ and the sign of the coefficient of $z^{2}$ is negative. Solving $C S^{N T}\left(\bar{x}_{R}^{L}\right)-C S^{E T}\left(x_{R}^{E T L}\right)=0$ with respect to $z$, we have two roots:

$$
z^{C S^{\prime}} \equiv \frac{-\Phi_{1}^{C S}+\sqrt{\left(\Phi_{1}^{C S}\right)^{2}-4 \Phi_{2}^{C S} \Phi_{0}^{C S}}}{2 \Phi_{2}^{C S}}, \quad z^{C S} \equiv \frac{-\Phi_{1}^{C S}-\sqrt{\left(\Phi_{1}^{C S}\right)^{2}-4 \Phi_{2}^{C S} \Phi_{0}^{C S}}}{2 \Phi_{2}^{C S}} .
$$

Because of $\Phi_{2}^{C S}<0, \Phi_{1}^{C S}<0$, and $\Phi_{0}^{C S}>0$, we have $z^{C S^{\prime}}<0$ and $z^{C S^{\prime}}<z^{C S}$. Hence, we ignore the threshold value $z^{C S^{\prime}}$.

Here, we compare $z^{C S}$ with $\bar{z}_{M}^{L}$. Then, for $\alpha \in(0,1 / 4)$, we have $z^{C S}<\bar{z}_{M}^{L}$. Therefore, we have $C S^{N T}\left(\bar{x}_{R}^{L}\right) \geq C S^{E T}\left(x_{R}^{E T L}\right)$ if $z \leq z^{C S} ; C S^{N T}\left(\bar{x}_{R}^{L}\right)<C S^{E T}\left(x_{R}^{E T L}\right)$ if $z^{C S}<z<\bar{z}_{M}^{L}$. 
Case (ii) For $\alpha \in[1 / 4,0.263)$ and $z \in\left[\bar{z}_{M}^{L}, \bar{z}_{M}^{H}\right)$, first, we compare $T S^{E T}\left(x_{R}^{E T L}\right)$ with $T S^{N T}\left(x_{R}^{N T}\right)$. The difference $T S^{E T}\left(x_{R}^{E T L}\right)-T S^{N T}\left(x_{R}^{N T}\right)$ is

$$
T S^{E T}\left(x_{R}^{E T L}\right)-T S^{N T}\left(x_{R}^{N T}\right)=\Phi_{2}^{T S} z^{2}+\Phi_{1}^{T S} z+\Phi_{0}^{T S}
$$

where

$$
\Phi_{2}^{T S} \equiv \frac{11-\alpha(2+5 \alpha)}{8(1-\alpha)^{2}}, \quad \Phi_{1}^{T S} \equiv-\frac{1-3 \alpha}{4(1-\alpha)}, \quad \Phi_{0}^{T S} \equiv-\frac{\alpha(24-11 \alpha)}{8(4-\alpha)^{2}}
$$

The difference is a quadratic function of $z$ and the sign of coefficient of $z^{2}$ is positive. Solving $T S^{E T}\left(x_{R}^{E T L}\right)-T S^{N T}\left(x_{R}^{N T}\right)=0$ with respect to $z$, we have two roots:

$$
z^{T S} \equiv \frac{-\Phi_{1}^{T S}+\sqrt{\left(\Phi_{1}^{T S}\right)^{2}-4 \Phi_{2}^{T S} \Phi_{0}^{T S}}}{2 \Phi_{2}^{T S}}, \quad z^{T S^{\prime}} \equiv \frac{-\Phi_{1}^{T S}-\sqrt{\left(\Phi_{1}^{T S}\right)^{2}-4 \Phi_{2}^{T S} \Phi_{0}^{T S}}}{2 \Phi_{2}^{T S}} .
$$

Because of $\Phi_{2}^{T S}>0, \Phi_{1}^{T S}<0$, and $\Phi_{0}^{T S}<0$, we have $z^{T S^{\prime}}<z^{T S}$. Moreover, for $\alpha \in$ $[1 / 4,0.263)$, we can numerically show $z^{T S^{\prime}}<0$. Hence, we ignore the threshold value $z^{T S^{\prime}}$. Comparing $z^{T S}$ with $\bar{z}_{M}^{L}$ and $\bar{z}_{M}^{H}$, we have $\bar{z}_{M}^{L}<z^{T S}<\bar{z}_{M}^{H}$ if $\alpha \in[1 / 4,0.2626) ; \bar{z}_{M}^{L}<\bar{z}_{M}^{H}<$ $z^{T S}$ if $\alpha \in[0.2626,0.263) .{ }^{13}$ Therefore, $T S^{E T}\left(x_{R}^{E T L}\right) \leq T S^{N T}\left(x_{R}^{N T}\right)$ if $\alpha \in[0.2626,0.263)$ or $\alpha \in[1 / 4,0.2626)$ and $z \in\left[\bar{z}_{M}^{L}, z^{T S}\right] ; T S^{E T}\left(x_{R}^{E T L}\right)>T S^{N T}\left(x_{R}^{N T}\right)$ if $\alpha \in[1 / 4,0.2626)$ and $z \in\left(z^{T S}, \bar{z}_{M}^{H}\right)$.

The other cases We consider the other cases: (I) $\alpha \in[0,1 / 4)$ and $z \in\left(0, \bar{z}_{M}^{L}\right)$ (note that the comparison for the consumer surplus is already done), (II) $\alpha \in[0,1 / 4)$ and $z \in\left[\bar{z}_{M}^{L}, z^{L}\right)$, (III) $\alpha \in[0,0.159)$ and $z \in\left[\bar{z}_{M}^{H}, z^{H}\right),(\mathrm{IV}) \alpha \in[0.159,1 / 5)$ and $z \in\left[z_{L}^{E T}, z^{H}\right),(\mathrm{V}) \alpha \in$ $[1 / 5,0.216)$ and $z \in\left[z_{L}^{E T}, z^{M}\right),(\mathrm{VI}) \alpha \in[0,1 / 5)$ and $z \in\left[z^{H}, z^{x R H}\right)$, (VII) $\alpha \in[1 / 5,0.216)$ and $z \in\left[z^{M}, z^{x R M}\right)$, (VIII) $\alpha \in[0.216,0.247)$ and $z \in\left[z_{L}^{E T}, z^{x R M}\right)$, (IX) $\alpha \in[0.159,0.216)$ and $z \in\left[\bar{z}_{M}^{H}, z_{L}^{E T}\right),(\mathrm{X}) \alpha \in[0.216,1 / 4)$ and $z \in\left[\bar{z}_{M}^{H}, z^{M}\right)$, (XI) $\alpha \in[0.216,0.247)$ and $z \in\left[z^{M}, z_{L}^{E T}\right),(\mathrm{XII}) \alpha \in[0.247,1 / 4)$ and $z \in\left[z^{M}, z^{x R M}\right)$, and (XIII) $\alpha \in[1 / 4,0.263)$ and $z \in\left[\bar{z}_{M}^{L}, \bar{z}_{M}^{H}\right)$ (note that the comparison for the total surplus is already done).

First, we show that for $\alpha \in[0,1 / 4)$ and $z \in\left(0, \bar{z}_{M}^{L}\right), \pi_{M}^{N T}\left(\bar{x}_{R}^{L}\right)>\pi_{M}^{E T}\left(x_{R}^{E T L}\right)$. The difference $\pi_{M}^{N T}\left(\bar{x}_{R}^{L}\right)-\pi_{M}^{E T}\left(x_{R}^{E T L}\right)$ is a quadratic function of $z$ and the sign of the coefficient

\footnotetext{
13 The value of 0.2626 is obtained by numerically solving $z^{T S}=\bar{z}_{M}^{H}$.
} 
of $z^{2}$ is negative. Solving $\pi_{M}^{N T}\left(\bar{x}_{R}^{L}\right)-\pi_{M}^{E T}\left(x_{R}^{E T L}\right)=0$ with respect to $z$, we have the roots: $z^{S o l 1}$ and $z^{S o l 2}$, where $z^{\text {Sol1 }} \leq z^{\text {Sol2 }}$. Comparing the threshold values $0, \bar{z}_{M}^{L}, z^{S o l 1}$, and $z^{S o l 2}$, we have $\left(0, \bar{z}_{M}^{L}\right) \subset\left[z^{S o l 1}, z^{S o l 2}\right]$. Because the coefficient of $z^{2}$ is negative, we obtain $\pi_{M}^{N T}\left(\bar{x}_{R}^{L}\right)>\pi_{M}^{E T}\left(x_{R}^{E T L}\right)$.

For the other cases, we employ similar procedures. Because we assume $z \in(0,1)$ in each case, we can consider a bounded interval for $z$. We denote it by $\left[z^{\text {low }}, z^{\text {high }}\right)$. In addition, we denote the outcome in Section 4.4 by $V^{R \& D}$ and that in Section 4.5 by $V^{E}$. Then, the difference $V^{R \& D}-V^{E}$ is a quadratic function of $z$ and the coefficient of $z^{2}$ consists of only $\alpha$. Because we assume $\alpha \in[0,1]$, we can determine the sign of coefficient of $z^{2}$ by a numerical calculation. Next, we calculate the sign of the discriminant of $V^{R \& D}-V^{E}=0$. If the discriminant is negative, we can find the sign of the difference $V^{R \& D}-V^{E}$ is the same as that of the coefficient of $z^{2}$. On the other hand, if the discriminant is non-negative, there exist one or two roots solving $V^{R \& D}-V^{E}=0$. We denote the roots by $z^{\text {Sol1 }}$ and $z^{\text {Sol2 }}$, where $z^{\text {Sol1 }}<z^{\text {Sol2 }}$. Finally, we can show that either $\left[z^{l o w}, z^{\text {high }}\right) \subset\left[z^{\text {Sol1 }}, z^{\text {Sol2 }}\right]$ or $\left[z^{\text {low }}, z^{\text {high }}\right) \subset\left(0, z^{\text {Sol1 }}\right] \cup\left[z^{\text {Sol2 }}, 1\right)$ must be satisfied. This result means that the sings of the difference $V^{R \& D}-V^{E}$ do not change. In addition, we can show that if the difference is zero, $z$ must be equal to one of the threshold values for $z: \bar{z}_{M}^{L}, \bar{z}_{M}^{H}, z^{L}, z^{M}, z^{H}, z^{x R M}, z^{x R H}$, and $z_{L}^{E T}$.

After some calculations, we obtain the following results; (I) for $\alpha \in[0,1 / 4)$ and $z \in$ $\left(0, \bar{z}_{M}^{L}\right)$, we have $\pi_{M}^{N T}\left(\bar{x}_{R}^{L}\right)>\pi_{M}^{E T}\left(x_{R}^{E T L}\right), \pi_{R}^{N T}\left(\bar{x}_{R}^{L}\right)>\pi_{R}^{E T}\left(x_{R}^{E T L}\right)$, and $T S^{N T}\left(\bar{x}_{R}^{L}\right)>T S^{E T}\left(x_{R}^{E T L}\right)$ (see Case (i) for the comparison between $C S^{N T}\left(\bar{x}_{R}^{L}\right)$ and $C S^{E T}\left(x_{R}^{E T L}\right)$ ); (II) for $\alpha \in[0,1 / 4$ ) and $z \in\left[\bar{z}_{M}^{L}, z^{L}\right)$, we have $\pi_{M}^{N T}\left(\bar{x}_{R}^{L}\right)>\pi_{M}^{N T}\left(x_{R}^{N T}\right), \pi_{R}^{N T}\left(\bar{x}_{R}^{L}\right)<\pi_{R}^{N T}\left(x_{R}^{N T}\right), C S^{N T}\left(\bar{x}_{R}^{L}\right)>$ $C S^{N T}\left(x_{R}^{N T}\right)$ and $T S^{N T}\left(\bar{x}_{R}^{L}\right)>T S^{N T}\left(x_{R}^{N T}\right)$; (III) for $\alpha \in[0,0.159)$ and $z \in\left[\bar{z}_{M}^{H}, z^{H}\right)$, we have $\pi_{M}^{N T}\left(x_{R}^{N T}\right) \leq \pi_{M}^{E T}\left(x_{R}^{E T M}\right), \pi_{R}^{N T}\left(x_{R}^{N T}\right) \geq \pi_{R}^{E T}\left(x_{R}^{E T M}\right), C S^{N T}\left(x_{R}^{N T}\right)<C S^{E T}\left(x_{R}^{E T M}\right)$, and $T S^{N T}\left(x_{R}^{N T}\right)<T S^{E T}\left(x_{R}^{E T M}\right)$, where the equality is satisfied at $z=\bar{z}_{M}^{H}$; (IV) for $\alpha \in$ $[0.159,1 / 5)$ and $z \in\left[z_{L}^{E T}, z^{H}\right)$, we have $\pi_{M}^{N T}\left(x_{R}^{N T}\right)<\pi_{M}^{E T}\left(x_{R}^{E T M}\right), \pi_{R}^{N T}\left(x_{R}^{N T}\right)>\pi_{R}^{E T}\left(x_{R}^{E T M}\right)$, $C S^{N T}\left(x_{R}^{N T}\right)<C S^{E T}\left(x_{R}^{E T M}\right)$, and $T S^{N T}\left(x_{R}^{N T}\right)<T S^{E T}\left(x_{R}^{E T M}\right) ;(\mathrm{V})$ for $\alpha \in[1 / 5,0.216)$ and $z \in\left[z_{L}^{E T}, z^{M}\right)$, we have $\pi_{M}^{N T}\left(x_{R}^{N T}\right)<\pi_{M}^{E T}\left(x_{R}^{E T M}\right), \pi_{R}^{N T}\left(x_{R}^{N T}\right)>\pi_{R}^{E T}\left(x_{R}^{E T M}\right), C S^{N T}\left(x_{R}^{N T}\right)<$ 
$C S^{E T}\left(x_{R}^{E T M}\right)$, and $T S^{N T}\left(x_{R}^{N T}\right)<T S^{E T}\left(x_{R}^{E T M}\right) ;(\mathrm{VI})$ for $\alpha \in[0,1 / 5)$ and $z \in\left[z^{H}, z^{x R H}\right)$, we have $\pi_{M}^{N T}\left(\bar{x}_{R}^{H}\right)<\pi_{M}^{E T}\left(x_{R}^{E T M}\right), \pi_{R}^{N T}\left(\bar{x}_{R}^{H}\right)>\pi_{R}^{E T}\left(x_{R}^{E T M}\right), C S^{N T}\left(\bar{x}_{R}^{H}\right)<C S^{E T}\left(x_{R}^{E T M}\right)$, and $T S^{N T}\left(\bar{x}_{R}^{H}\right)<T S^{E T}\left(x_{R}^{E T M}\right)$; (VII) for $\alpha \in[1 / 5,0.216)$ and $z \in\left[z^{M}, z^{x R M}\right)$, we have $\pi_{M}^{N T}\left(\bar{x}_{R}^{M}\right)<\pi_{M}^{E T}\left(x_{R}^{E T M}\right), \pi_{R}^{N T}\left(\bar{x}_{R}^{M}\right)>\pi_{R}^{E T}\left(x_{R}^{E T M}\right), C S^{N T}\left(\bar{x}_{R}^{M}\right)<C S^{E T}\left(x_{R}^{E T M}\right)$, and $T S^{N T}\left(\bar{x}_{R}^{M}\right)<$ $T S^{E T}\left(x_{R}^{E T M}\right) ;(\mathrm{VIII})$ for $\alpha \in[0.216,0.247)$ and $z \in\left[z_{L}^{E T}, z^{x R M}\right)$, we have $\pi_{M}^{N T}\left(\bar{x}_{R}^{M}\right)<$ $\pi_{M}^{E T}\left(x_{R}^{E T M}\right), \pi_{R}^{N T}\left(\bar{x}_{R}^{M}\right)>\pi_{R}^{E T}\left(x_{R}^{E T M}\right), C S^{N T}\left(\bar{x}_{R}^{M}\right)<C S^{E T}\left(x_{R}^{E T M}\right)$, and $T S^{N T}\left(\bar{x}_{R}^{M}\right)<T S^{E T}\left(x_{R}^{E T M}\right) ;$ (IX) for $\alpha \in[0.159,0.216)$ and $z \in\left[\bar{z}_{M}^{H}, z_{L}^{E T}\right)$, we have $\pi_{M}^{N T}\left(x_{R}^{N T}\right) \leq \pi_{M}^{E T}\left(x_{R}^{E T L}\right), \pi_{R}^{N T}\left(x_{R}^{N T}\right) \geq$ $\pi_{R}^{E T}\left(x_{R}^{E T L}\right), C S^{N T}\left(x_{R}^{N T}\right)<C S^{E T}\left(x_{R}^{E T L}\right)$, and $T S^{N T}\left(x_{R}^{N T}\right)<T S^{E T}\left(x_{R}^{E T L}\right)$, where the equality is satisfied at $z=\bar{z}_{M}^{H} ;(\mathrm{X})$ for $\alpha \in[0.216,1 / 4)$ and $z \in\left[\bar{z}_{M}^{H}, z^{M}\right)$, we have $\pi_{M}^{N T}\left(x_{R}^{N T}\right) \leq$ $\pi_{M}^{E T}\left(x_{R}^{E T L}\right), \pi_{R}^{N T}\left(x_{R}^{N T}\right) \geq \pi_{R}^{E T}\left(x_{R}^{E T L}\right), C S^{N T}\left(x_{R}^{N T}\right)<C S^{E T}\left(x_{R}^{E T L}\right)$, and $T S^{N T}\left(x_{R}^{N T}\right)<$ $T S^{E T}\left(x_{R}^{E T L}\right)$, where the equality is satisfied at $z=\bar{z}_{M}^{H}$; (XI) for $\alpha \in[0.216,0.247)$ and $z \in\left[z^{M}, z_{L}^{E T}\right)$, we have $\pi_{M}^{N T}\left(\bar{x}_{R}^{M}\right)<\pi_{M}^{E T}\left(x_{R}^{E T L}\right), \pi_{R}^{N T}\left(\bar{x}_{R}^{M}\right)>\pi_{R}^{E T}\left(x_{R}^{E T L}\right), C S^{N T}\left(\bar{x}_{R}^{M}\right)<$ $C S^{E T}\left(x_{R}^{E T L}\right)$ and $T S^{N T}\left(\bar{x}_{R}^{M}\right)<T S^{E T}\left(x_{R}^{E T L}\right)$; (XII) for $\alpha \in[0.247,1 / 4)$ and $z \in\left[z^{M}, z^{x R M}\right)$, we have $\pi_{M}^{N T}\left(\bar{x}_{R}^{M}\right)<\pi_{M}^{E T}\left(x_{R}^{E T L}\right), \pi_{R}^{N T}\left(\bar{x}_{R}^{M}\right)>\pi_{R}^{E T}\left(x_{R}^{E T L}\right), C S^{N T}\left(\bar{x}_{R}^{M}\right)<C S^{E T}\left(x_{R}^{E T L}\right)$ and $T S^{N T}\left(\bar{x}_{R}^{M}\right)<T S^{E T}\left(x_{R}^{E T L}\right) ;(\mathrm{XIII})$ for $\alpha \in[1 / 4,0.263)$ and $z \in\left[\bar{z}_{M}^{L}, \bar{z}_{M}^{H}\right)$, we have $\pi_{M}^{E T}\left(x_{R}^{E T L}\right)<$ $\pi_{M}^{N T}\left(x_{R}^{N T}\right), \pi_{R}^{E T}\left(x_{R}^{E T L}\right)<\pi_{R}^{N T}\left(x_{R}^{N T}\right)$, and $C S^{E T}\left(x_{R}^{E T L}\right)>C S^{N T}\left(x_{R}^{N T}\right)$ (see Case (ii) for the comparison between $T S^{E T}\left(x_{R}^{E T L}\right)$ and $\left.T S^{N T}\left(x_{R}^{N T}\right)\right)$.

Summarizing the above discussion, we have the following result: (a) commitment to either encroachment or non-encroachment (simply call it the commitment decision) strictly decreases the profits of $M$ and $R$, the consumer surplus, and the total surplus if $\alpha \in[0,1 / 4)$ and $z \in\left(0, z^{C S}\right)$; (b) the commitment decision strictly decreases the profits of $M$ and $R$ and the total surplus, and strictly increases the consumer surplus if $\alpha \in[0,1 / 4)$ and $z \in\left(z^{C S}, \bar{z}_{M}^{L}\right) ;(\mathrm{c})$ the commitment decision strictly decreases the profit of $M$, the consumer surplus, and the total surplus, and strictly increases the profit of $R$ if $\alpha \in[0,1 / 4)$ and $z \in\left(\bar{z}_{M}^{L}, z^{L}\right) ;(\mathrm{d})$ the commitment decision strictly increases the profit of $M$, the consumer surplus, and the total surplus, and strictly decreases the profit of $R$ if $\alpha \in[0,1 / 5)$ and $z \in\left(\bar{z}_{M}^{H}, z^{x R H}\right)$ or $\alpha \in[1 / 5,1 / 4)$ and $z \in\left(\bar{z}_{M}^{H}, z^{x R M}\right)$; (e) the commitment decision strictly increases the profits of $M$ and $R$ and the total surplus, and strictly decreases the consumer 
surplus if $\left(\alpha \in[1 / 4,0.2626)\right.$ and $\left.z \in\left(\bar{z}_{M}^{L}, z^{T S}\right)\right)$ or $\left(\alpha \in[0.2626,0.263)\right.$ and $\left.z \in\left(\bar{z}_{M}^{L}, \bar{z}_{M}^{H}\right)\right)$. (f) the commitment decision strictly increases the profits of $M$ and $R$, and strictly decreases the consumer surplus and the total surplus if $\alpha \in[1 / 4,0.2626)$ and $z \in\left(z^{T S}, \bar{z}_{M}^{H}\right)$. Therefore, we obtain Proposition 6. 